\title{
Radiometric Calibration Targets for the Mastcam-Z Camera on the Mars 2020 Rover Mission
}

\author{
K.M. Kinch ${ }^{1}$ (D) M.B. Madsen ${ }^{1}$ - J.F. Bell III ${ }^{2}$ J.N. Maki ${ }^{3}$ - Z.J. Bailey ${ }^{3}$ A.G. Hayes ${ }^{4}$. \\ O.B. Jensen ${ }^{1}$ - M. Merusi ${ }^{1}$ - M.H. Bernt ${ }^{1}$ - A.N. Sørensen ${ }^{1}$ - M. Hilverda ${ }^{5}$ - E. Cloutis ${ }^{6}$. \\ D. Applin' ${ }^{6}$ E. Mateo-Marti ${ }^{7}$. J.A. Manrique ${ }^{8}$ - G. Lopez-Reyes ${ }^{8}$ - A. Bello-Arufe9 . \\ B.L. Ehlmann ${ }^{3,10} \cdot$ J. Buz $^{11}$ • A. Pommerol ${ }^{12}$ - N. Thomas ${ }^{12}$ - L. Affolter ${ }^{12}$. \\ K.E. Herkenhoff ${ }^{13}$ - J.R. Johnson ${ }^{14}$ - M. Rice ${ }^{15}$ - P. Corlies ${ }^{4}$ C. Tate $^{4}$.

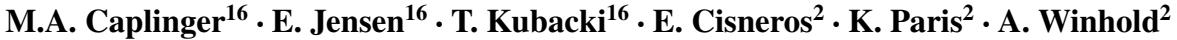

Received: 18 May 2020 / Accepted: 17 November 2020 / Published online: 3 December 2020

(C) The Author(s) 2020, corrected publication 2021

Abstract The Mastcam-Z Camera is a stereoscopic, multispectral camera with zoom capability on NASA's Mars-2020 Perseverance rover. The Mastcam-Z relies on a set of two deck-mounted radiometric calibration targets to validate camera performance and to provide an instantaneous estimate of local irradiance and allow conversion of image data to units of reflectance $\left(\mathrm{R}^{*}\right.$ or $\left.\mathrm{I} / \mathrm{F}\right)$ on a tactical timescale. Here, we describe the heritage, design, and

The Mars 2020 Mission

Edited by Kenneth A. Farley, Kenneth H. Williford and Kathryn M. Stack

K.M. Kinch

kinch@nbi.ku.dk

1 Niels Bohr Institute, University of Copenhagen, Copenhagen, Denmark

2 School of Earth and Space Exploration, Arizona State University, Tempe, AZ, USA

3 Jet Propulsion Laboratory, California Institute of Technology, Pasadena, CA, USA

4 Department of Astronomy, Cornell University, Ithaca, NY, USA

5 The Planetary Society, Pasadena, CA, USA

6 Department of Geography, University of Winnipeg, Winnipeg, Manitoba, Canada

7 Centro de Astrobiología (CSIC-INTA), Torrejón de Ardoz, Madrid, Spain

8 University of Valladolid, Valladolid, Spain

9 National Space Institute, Technical University of Denmark, Lyngby, Denmark

10 Division of Geological and Planetary Sciences, California Institute of Technology, Pasadena, CA, USA

11 Department of Astronomy and Planetary Science, Northern Arizona University, Flagstaff, AZ, USA

12 Space and Planetary Science Department, Physikalisches Institut, University of Bern, Bern, Switzerland

13 Astrogeology Science Center, United States Geological Survey, Flagstaff, AZ, USA

14 Johns Hopkins University Applied Physics Laboratory, Laurel, MD, USA 
optical characterization of these targets and discuss their use during rover operations. The Mastcam-Z primary calibration target inherits features of camera calibration targets on the Mars Exploration Rovers, Phoenix and Mars Science Laboratory missions. This target will be regularly imaged during flight to accompany multispectral observations of the martian surface. The primary target consists of a gold-plated aluminum base, eight strong hollowcylinder $\mathrm{Sm}_{2} \mathrm{Co}_{17}$ alloy permanent magnets mounted in the base, eight ceramic color and grayscale patches mounted over the magnets, four concentric, ceramic grayscale rings and a central aluminum shadow post (gnomon) painted with an IR-black paint. The magnets are expected to keep the central area of each patch relatively free of Martian aeolian dust. The Mastcam- $Z$ secondary calibration target is a simple angled aluminum shelf carrying seven vertically mounted ceramic color and grayscale chips and seven identical, but horizontally mounted ceramic chips. The secondary target is intended to augment and validate the calibration-related information derived from the primary target. The Mastcam- $Z$ radiometric calibration targets are critically important to achieving Mastcam-Z science objectives for spectroscopy and photometric properties.

\section{Introduction}

NASA's Mars 2020 Perseverance Rover mission (Farley et al. 2020, this issue) will launch for Mars in July 2020 and land in Jezero Crater in February of 2021. The Mastcam-Z Camera (Bell et al. 2021) is a stereoscopic, multispectral camera with zoom capability and is mounted on the rover mast. Mastcam- $Z$ relies on a set of radiometric calibration targets to provide an instantaneous estimate of local illumination conditions and thus convert images of the Martian surface from units of radiance (the instrument observable) to units of reflectance (the material property) on a tactical timescale. There are two targets, the primary and the secondary Mastcam- $Z$ radiometric calibration targets. Both are mounted on the rover deck and viewable from Mastcam-Z. Both targets were designed, built and delivered by the Niels Bohr Institute of the University of Copenhagen.

In this manuscript we describe in detail the design and characterization of the targets, and discuss the plans for operational imaging of the targets and processing of such images for the purpose of generating in-flight reflectance ( $\mathrm{I} / \mathrm{F})$-calibrated Mastcam- $\mathrm{Z}$ image products. This manuscript is part of a set of 3 papers describing the Mastcam- $Z$ investigation. The paper by Bell et al. (2021) gives an overview of the Mastcam-Z investigation, its goals and components while the paper by Hayes et al. (2021) describes in detail the pre-flight calibration and performance of the flight Mastcam-Z instrument.

Section 2 describes briefly the Mars 2020 mission and Mastcam-Z instrument, gives a more detailed overview of the purpose of the calibration targets and describes their extensive heritage from previous missions. Section 3 describes the design of the two targets. Section 4 presents data from pre-flight spectroscopic characterization of assembled targets as well as "witness samples" of the color and grayscale reference materials embedded in the targets. Section 5 discusses in-flight use of the targets as well as the process for generating in-flight reflectance-calibrated (I/F) Mastcam-Z image products, and Secton 6 gives a summary. Appendix A describes the materials and methods used for construction of more than 20 calibration target units produced (engineering models, flight units, flight spares, display models and "calibration" units). Appendix B describes the testing procedures and results from the dynamical and thermal test programs.

15 College of Science and Engineering, Western Washington University, Bellingham, WA, USA

16

Malin Space Science Systems, San Diego, CA, USA 

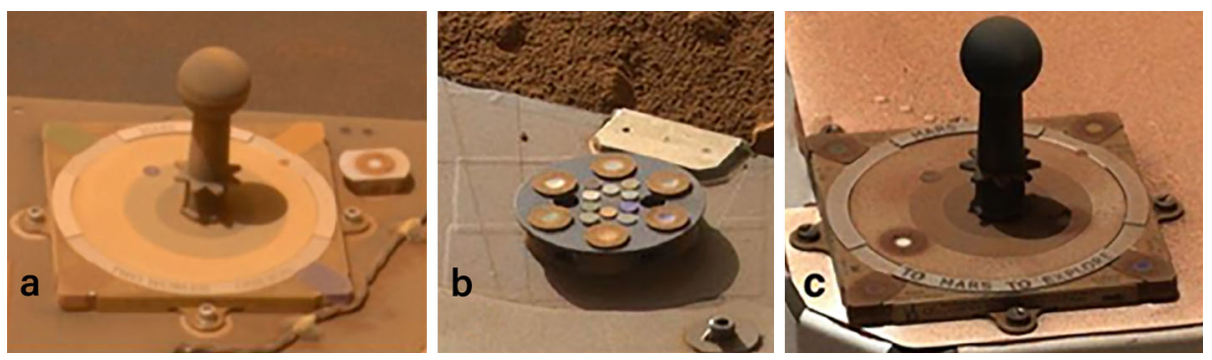

Fig. 1 (a) Pancam calibration target on the Mars Exploration Rover (MER) Spirit, sol 711, sweep magnet visible to the right of the main target. (b) One of three Surface Stereo Imager calibration targets on the Phoenix lander, sol 106. (c) The Mastcam calibration target on the Mars Science Laboratory (MSL) rover, sol 705

\section{Background}

\subsection{Mars 2020 and Mastcam-Z}

NASA's Perseverance Rover (Farley et al. 2020, this issue) is a large rover similar to the currently active "Curiosity" rover (e.g., Grotzinger et al. 2014, and 2015). A central objective is to select and collect a compelling set of samples of the Martian surface. A follow-up joint ESA-NASA mission will later return these samples to Earth. This makes the 2020 mission the first step in a two-step process that will achieve Mars sample return, a major and long-standing goal of the Mars research community (e.g., Bogard et al. 1979), and a huge undertaking.

The Mastcam-Z (Bell et al. 2021) is a mast-mounted stereo camera with zoom capability. Mastcam- $Z$ acquires multispectral observations through 11 different narrow-band filters spanning $450 \mathrm{~nm}-1000 \mathrm{~nm}$ plus a standard set of red, green and blue Bayer broadband filters (Hayes et al. 2021). This capability will help the rover map compositional variations across the local terrain and bridge the gap between hyperspectral imaging from orbit and detailed mineralogical and geochemical point measurements by other instruments on the payload. Reflectance spectroscopy is generally unable to provide a full quantitative mineralcompositional analysis, but can identify the presence of specific important minerals - or classes of minerals - and track more general features such as e.g., redox state of iron or the presence of hydration in rocks and soils.

\subsection{Purpose of Calibration Targets}

The Mastcam-Z primary radiometric calibration target is a high-heritage (Fig. 1), evolutionary development that combines design elements from camera calibration targets on the Mars Exploration Rovers (MER), Phoenix, and Mars Science Laboratory (MSL) missions.

The Mastcam-Z primary calibration target (Fig. 2) is mounted on the rover deck (Fig. 3) on top of the Rover Pyro Firing Assembly (RPFA) and will be used routinely by Mastcam-Z to (a) verify and validate preflight calibration after the instruments have arrived on Mars, (b) assist in tactical conversion of images of the Martian surface from radiance to reflectance by comparison with calibration target surfaces of known reflectance, (c) estimate the ratio of irradiance in the direct solar beam to diffuse sky irradiance reaching the surface via atmospheric scatterings and (d) monitor the stability of the calibration throughout the mission. Of these, arguably (b) is the most important and the crucial justification for the inclusion of 


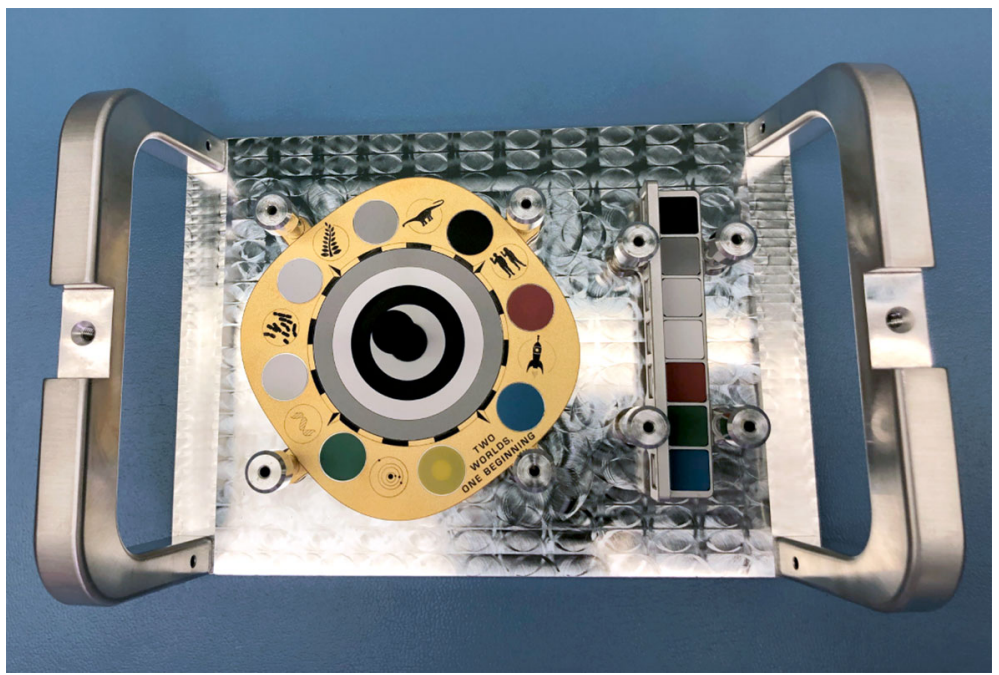

Fig. 2 Flight Mastcam-Z calibration targets mounted on their transport bracket prior to delivery in May 2019. Left side: Primary target, Right side: Secondary target. The total height of the primary target is 45.5 $\mathrm{mm}$ while the base of the target fits inside a square with a side of $98 \mathrm{~mm}$. The secondary target fits inside a box $80 \mathrm{~mm} \times 30 \mathrm{~mm} \times 16 \mathrm{~mm}$
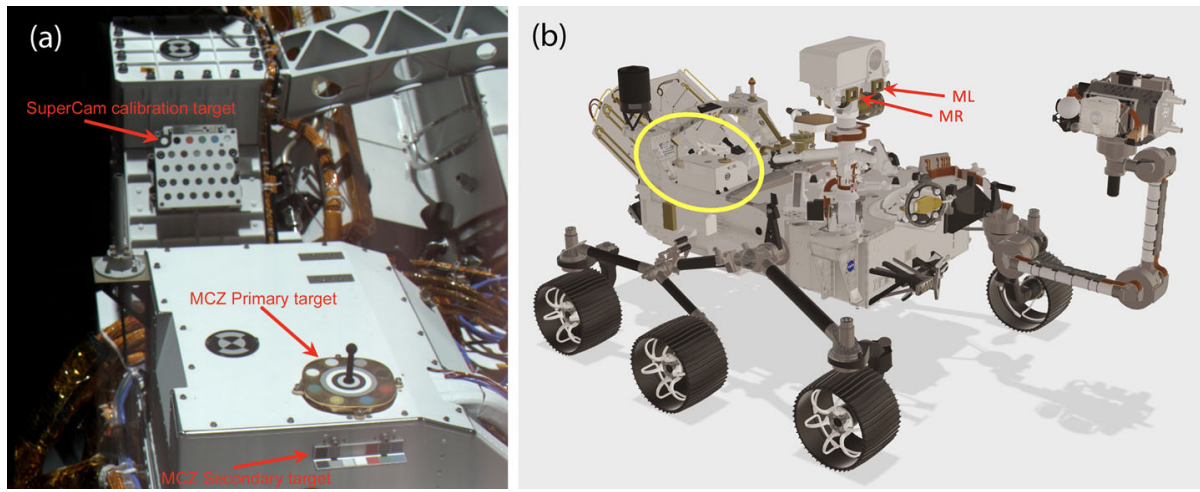

Fig. 3 a) Flight Mastcam-Z calibration targets mounted on the rover and imaged using flight cameras (L0 filter) during Assembly Test and Launch Operations in October 2019. The SuperCam calibration target is also visible in the frame. b) Technical drawing of the rover with the two camera eyes pointed out with red arrows and the scene of frame a) circled in yellow

the target. The illumination conditions on the Martian surface change with time of year, time of day, and with local atmospheric conditions such as dust loading and potentially the presence of clouds. The local atmospheric conditions may change on short timescales and are hard to model accurately. The in-situ presence of a well-characterized reference provides a local, instantaneous estimate of illumination conditions and thus allows for reliable tacticaltimescale transformation of image data from radiance to reflectance. The Mastcam-Z Flight Calibration Target is critically important to achieving Mastcam- $Z$ science objectives that center on color, spectroscopy and photometric properties (e.g., Bell et al. 2021). 
The secondary target (Fig. 2) is mounted in a location (Fig. 3) right below the primary target on the vertical front face of the RPFA box on which the primary target is mounted. This positions the secondary target in a different dust deposition environment from the primary target but will still allow imaging of the secondary target in the same frame as primary target images.

The primary motivation for the addition of the Mastcam-Z secondary calibration target springs from two specific observations on the MSL (Curiosity Rover) mission:

First, there was significant deposition of dust (and indeed also sand-sized particles) on the rover deck during landing. This dust was mobilized from the local area by the skycrane retrorockets and amounted to an optical depth of deposited material of a little more than 0.1. This is equivalent to $\sim 25$ sols worth of dust accumulation under moderately dusty conditions (Kinch et al. 2015). This dust precluded post-landing imaging of a clean calibration target, and complicated dust correction procedures by introducing a dust population inherited from local rocks/soil and with unknown spectral properties distinct from those of global atmospheric Martian dust.

The second observation is that the location of the MSL Mastcam calibration target over time became one of the dustier regions of the rover deck. The location of the Mars-2020 Mastcam-Z primary calibration target, while not identical, is in the same general area of the rover deck and so might be expected to see significant dust accumulation during the mission as well. Based on the experience from Curiosity the dust deposition environment at the secondary target is expected to be more benign both during landing and during surface operations.

The purpose of the Mastcam-Z Flight Secondary Calibration Target is to (a) act as a cross-check with the main calibration target at a location likely to be more shielded from dust deposition during landing and during later operation, (b) monitor dust deposition without the effects of magnets as are present in the primary target and (c) by having both horizontally and vertically mounted surfaces allow testing of the potential role of calibration target photometric effects in estimates of derived reflectance.

\subsection{Heritage}

Since Viking, all Mars lander missions have carried some kind of camera color reference (Huck et al. 1975; Smith et al. 1997). The Mastcam-Z primary calibration target traces direct heritage to the Mars Exploration Rover, Phoenix and Mars Science Laboratory missions. Figure 1 shows images of calibration targets on these three previous Mars surface missions. All rely on a small set of colored Room-Temperature Vulcanized (RTV) silicone rubber patches with well-characterized reflectance spectra. The MER Pancam target (Bell et al. 2003) and the MSL Mastcam target (Bell et al. 2017) each have 7 color and grayscale patches while the Phoenix Surface stereo Imager (SSI) target (Leer et al. 2008) has 6 main patches (plus 6 small patches and 4 experimental patches in the central area).

Previous experiments with permanent magnets (Hargraves et al. 1976, and 1979; Bertelsen et al. 2004) have shown that a substantial fraction of airborne dust particles in the Martian atmosphere are sufficiently magnetic to be attracted by strong permanent magnets. The MER Pancam calibration target included a separate "sweep magnet" experiment (Bertelsen et al. 2004; Madsen et al. 2009) which demonstrated the capability of a fixed, permanent strong hollow-cylinder magnet to keep a small area relatively free of airfall dust. Subsequently 6 such magnets were incorporated in both the Phoenix (Leer et al. 2008; Drube et al. 2010) and MSL (Kinch et al. 2013) targets in order to reduce dust deposition on certain regions. The MER and MSL targets both include a shadow post (gnomon) to estimate the relative intensity of diffuse sky irradiance versus irradiance in the direct solar beam. 


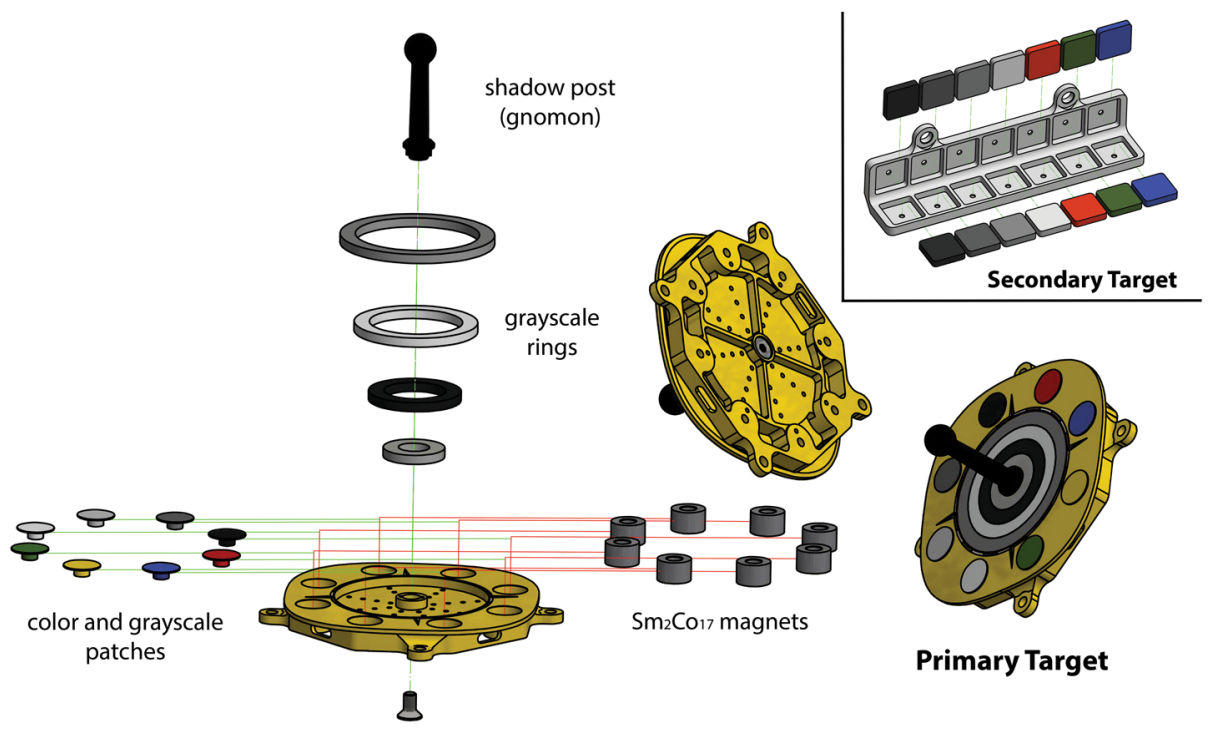

Fig. 4 Technical drawing of the separate parts of the primary and secondary calibration targets, illustrating assembly, assembled Mastcam-Z primary calibration target. The total height of the primary target is $45.5 \mathrm{~mm}$ while the base of the target fits inside a square with a side of $98 \mathrm{~mm}$. The secondary target fits inside a box $80 \mathrm{~mm} \times 30 \mathrm{~mm} \times 16 \mathrm{~mm}$. For detailed dimensions of individual components, see Sect. 3.1.1

Like these three previous calibration targets, the frame of the Mastcam-Z primary calibration target (Fig. 4) is constructed from aluminum. The surface is ball-blasted and treated with a silver and gold anodization ( $40 \mu \mathrm{m}$ layer of silver followed by a $3 \mu \mathrm{m}$ layer of gold). This is analogous to that used on the MER Pancam and MSL Mastcam targets. The gnomon is similar, though smaller, than the gnomon on the MER and MSL targets and painted with a similar IR-black paint.

The target contains 8 "sweep" magnets identical to the 6 magnets contained in the Phoenix calibration target. These are made from the same material, but are bigger than the magnets in the MSL Mastcam target and the original sweep magnet associated with the MER Pancam.

The materials for the optical patches, sintered alumina and glazed aluminum silicate, are different from the heritage material with significant advantages as detailed in Sect. 3 below. Sintered alumina and glazed aluminum silicate are also being employed in the design of the SuperCam calibration target for the Mars 2020 Rover (Manrique et al. 2020, this issue).

\section{Calibration Targets Design}

This section describes the design of the primary and secondary Mastcam- $Z$ radiometric calibration targets and gives justification for the design choices. Figure 2 shows a highresolution image of the flight calibration targets. Figure 3 shows their location on the rover and gives an idea of how the calibration targets will appear when viewed from Mastcam- $Z$ during flight. Figure 4 shows technical drawings of the separate parts illustrating assembly. 


\subsection{Primary Target}

The primary calibration target is the larger of the two targets and the one that will be used in the standard procedure for radiance-to-reflectance conversion of Mastcam-Z images. The primary target has explicit heritage from a series of preceding missions as described in Sect. 2.3.

\subsubsection{Design Overview}

The primary target mounts to the deck of the Perseverance rover with 4 fasteners arranged in a quadratic pattern that spans a diagonal distance of $90.0 \mathrm{~mm}$. The body of the target is constructed from aluminum with a silver and gold anodization. The central shadow post is aluminum painted with an IR-black paint (Aeroglaze Z307). The total height of the target is $45.5 \mathrm{~mm}$ while the base of the target fits inside a square with a side of $98 \mathrm{~mm}$. The total mass of the target is $103 \mathrm{~g}$.

There are four central grayscale rings and eight outer circular patches (four grayscales and four colors) that encompass the periphery of the calibration target.

The top surface of the patches has a diameter of $12 \mathrm{~mm}$ and a thickness of $0.4 \mathrm{~mm}$. The stub underneath has a diameter of $5 \mathrm{~mm}$ and a thickness of $3 \mathrm{~mm}$. The rings are all $3 \mathrm{~mm}$ thick. The innermost ring has an inner diameter of $10 \mathrm{~mm}$ and an outer diameter of $20 \mathrm{~mm}$, the second ring has an inner diameter of $20 \mathrm{~mm}$ and an outer diameter of $30 \mathrm{~mm}$, the third ring has an inner diameter of $30 \mathrm{~mm}$ and an outer diameter of $40 \mathrm{~mm}$ and the outermost ring has an inner diameter of $40 \mathrm{~mm}$ and an outer diameter of $50 \mathrm{~mm}$. Exact dimensions of the rings are very slightly different from what is listed above, in order to leave very slight gaps between the rings to allow for thermal expansion/contraction.

These patches and rings are manufactured from optically stable ceramic materials with a variety of reflectance characteristics. Their bidirectional reflectance was carefully characterized before launch (see Sect. 4). The 4 grayscales in the central rings are identical to the 4 grayscales in the circles around the periphery. The white ring and white patch are made from AluWhite98 sintered alumina provided by Avian technologies in the US (www.aviantechnologies.com). The rest (all colors, blacks, and greys) are made from glazed and matted aluminum-silicates provided by Lucideon in the UK (www.lucideon.com). The names of the 7 materials provided by Lucideon are Black, Grey33, Grey70, Cyan, Green, Bright Yellow and Red. Here, we refer to them as Black, Dark Grey, Light Grey, Blue, Green, Yellow and Red.

Underneath the 8 color and grayscale patches around the periphery are mounted 8 ringshaped $\mathrm{Sm}_{2} \mathrm{Co}_{17}$ alloy permanent magnets, magnetized along the axis of symmetry. These strong "sweep" magnets are designed to attract even weakly magnetic martian dust particles, thereby keeping these from accessing a small area in the center of each of the 8 patches. The magnets are hollow cylinders with outer diameter $11 \mathrm{~mm}$, inner diameter $5 \mathrm{~mm}$, and height $7 \mathrm{~mm}$. Based on simulation and on experience with similar magnets on previous missions (see e.g. Fig. 1c and Sect. 5.2) this clean surface is expected to have a diameter of $\sim 3 \mathrm{~mm}$.

The motto and 7 small graphics shown on the primary target in Fig. 2 are laser-engraved into the gold-and-silver anodization. They tell a story of life emerging on Earth and evolving into humans that then develop space flight. These outreach elements were developed in collaboration with the Planetary Society as the outreach partner for Mastcam-Z. They are discussed in more detail in Sect. 3.1.3.

The pattern of the 4 fastener holes is aligned with the rover $\mathrm{x}$ and $\mathrm{y}$ axes. The symmetry axes of the target itself are rotated by $7.72^{\circ}$ relative to this pattern in order to line up the 
symmetry axis of the target with the line of sight from the rover mast to the calibration target. The pattern around the outermost grayscale ring is designed to call to mind a compass rose or heading diagram. This pattern is aligned with the rover coordinate system.

The curve that defines the outer rim of the calibration target is a superellipse or Lamé curve. The superellipse is a smooth and mathematically elegant compromise between a circle and a square. It saves on mass relative to a square design using the same mounting, while retaining more available area than a circular design, which would force the circle of 8 patches closer together and reduce the available area in the center of the target.

\subsubsection{Changes from Heritage Designs}

While the design for the Mastcam-Z primary calibration target inherits many features from previous targets as described in Sect. 2.3, it also displays a number of features that are modifications of previous designs. The more prominent design modifications are listed below alongside the rationale for each change.

Color Patches from Ceramic Materials The color and grayscale patches and rings on the calibration target are produced from alumina sinters and glazed aluminum silicate sinters instead of the pigmented Room Temperature Vulcanized (RTV) silicone rubber materials used on previous targets. Advantages of this option are:

(1) Being sintered powders, their reflectance properties are closer to Lambertian than RTV which is a silicone rubber that naturally produces a relatively specular surface. RTV is molded in a mold prepared by glass bead blasting in order to produce a diffusively reflective surface - but even so the surface did display some specularity. As described in Sect. 4.2 the ceramic materials used for the Mastcam-Z targets are not perfectly Lambertian either, as particularly the darker materials do display a significant forward-scattering tendency.

(2) The patches are produced by high temperature processing of inorganic materials and the material used in these sintered materials offers substantially lower outgassing rates. Because of this, and since they are inorganic, they are not a potential source of organic contamination for other rover instruments.

(3) Alumina sinters and glazed aluminum silicate sinters are also used for the white IR and RGB reference patches in the SuperCam calibration target. Using similar material on the Mastcam-Z calibration target will benefit cross-calibration efforts and allow a sharing of experience that will optimize efficiency and minimize duplication of effort.

The main downside of the new materials is brittleness that proved a challenge during assembly (see Appendix A) and required some minor redesign after thermal testing (see Appendix B). Once assembled in the final design configuration, the thinnest of the ceramic surfaces are supported by epoxy layers below and the final design of the target has passed dynamical and thermal tests with no anomalies.

Bigger Magnets The "sweep" magnets (Fig. 4) used are hollow cylinders with outer diameter $11 \mathrm{~mm}$, inner diameter $5 \mathrm{~mm}$, height $7 \mathrm{~mm}$ and they produce a "clean spot" of roughly $3 \mathrm{~mm}$ diameter in the center of each magnet. The color chips are machined in a precise dimension and shape to fit into the magnets rather than magnets retrofitted into an already assembled target as was the case for the Mastcam target on Curiosity. This allows the magnets to be placed closer and at a more precisely known level under the optical surface. The magnets are identical to the design of the Phoenix magnets and larger (twice the 
volume) than the magnets mounted in the MSL Mastcam target. Each magnet weighs $3.9 \mathrm{~g}$. The larger magnets and improved mounting produce an area reasonably clean of dust that is roughly twice that produced by the smaller magnets. The larger clean area improves the statistics of the measurement of calibration target radiance by providing more clean pixels. Such magnets were flown with good results on Phoenix and extensively laboratory-tested in simulated Martian conditions before that mission. See Sect. 5.2 for details about the effect of the magnets on dust accumulation.

More Grayscales The target includes 4 grayscale rings and patches as compared to 3 in previous designs and the total span in reflectance of the grayscales is larger than previous designs. The larger span in reflectance results in a better radiance-reflectance fit. At certain wavelengths (e.g., MER Pancam L3 $(673 \mathrm{~nm})$ and R7 $(1009 \mathrm{~nm})$ ) the set of calibration target patches on the Pancam / Mastcam target tend to cluster in reflectance around a few values, reducing the quality of the radiance-reflectance fit. This problem is ameliorated with the new design, since the grayscales always provide at least 4 data points that span a wide range in reflectance values.

Order of Grayscale Rings The order of the grayscale rings is not monotonically increasing black-to-white. This has several advantages:

(1) The innermost ring potentially delivers data of slightly lower quality because it is most affected by shading and/or reflectance of light from the gnomon and because it has the fewest pixels. This innermost ring is now not the darkest or brightest grayscale, which, being endpoints on the scale, are most important.

(2) The shadow of the gnomon is used for evaluating the intensity of diffuse sky irradiance. Cases when the shadow does not reach all rings (high sun) now give a larger total span in grayscales than before.

(3) The contrast between any two rings is now larger than before, making selection easier even on images of a heavily dust-coated calibration target.

No Magnets in Central Rings Unlike on the MSL Mastcam target, no magnets are mounted within the central grayscale rings. This makes for a reasonably uniform dust layer on the central rings which improves the capability for evaluating the shadowed versus sunlit irradiance and allows a more reliable radiance-reflectance fit to data from the 4 grayscale rings. It will also make the target better for monitoring dust dynamics, although the presence of magnets beneath the 8 peripheral patches will still affect the distribution of dust. Note that the grayscales in the 4 rings are the same as the grayscales in 4 of the 8 peripheral magnetically-protected patches.

Smaller Gnomon The entire sequence of grayscale rings is downscaled relative to the MER/MSL calibration targets. This is compensated by the zoom capability and higher resolution of the Mastcam-Z, thus still allowing an adequate number of pixels for the calibration procedure. Since the purpose of the gnomon is to cast a shadow across the grayscale rings the smaller diameter of the rings allowed a downscaling of the gnomon as well. The gnomon weighs only $3 \mathrm{~g}$, less than a single magnet. The total height of the target is $45.5 \mathrm{~mm}$ and the top of the gnomon is $37.5 \mathrm{~mm}$ above the top surface of the target. The outer diameter of the outer grayscale ring is $50 \mathrm{~mm}$, which means that the gnomon shadow will reach exactly to the outer edge of the outermost grayscale ring when the solar incidence angle is 56 degrees. For higher solar elevation (lower incidence angle), fewer rings will be shadowed - but in 
Table 1 Deviations from heritage as detailed in Sect. 3.1.2

\begin{tabular}{|c|c|c|}
\hline Change from heritage & Justification & Risk \\
\hline $\begin{array}{l}\text { Ceramic color and } \\
\text { grayscale materials } \\
\text { instead of RTV }\end{array}$ & $\begin{array}{l}\text { Better Optical performance. } \\
\text { Lower outgassing }\end{array}$ & $\begin{array}{l}\text { Materials with low heritage. (Completed test } \\
\text { program mitigates associated risk) }\end{array}$ \\
\hline Bigger magnets & Better dust mitigation & Very low risk. (Heritage from Phoenix) \\
\hline More grayscales & Better radiance-reflectance fit & Extremely low risk \\
\hline $\begin{array}{l}\text { Changed order of } \\
\text { grayscales }\end{array}$ & $\begin{array}{l}\text { Better radiance-reflectance fit. } \\
\text { Better diffuse light estimate }\end{array}$ & Extremely low risk \\
\hline $\begin{array}{l}\text { No magnets in central } \\
\text { rings }\end{array}$ & $\begin{array}{l}\text { Simplification allowed by } \\
\text { larger number of dedicated } \\
\text { magnet-protected patches. } \\
\text { Better diffuse light estimate }\end{array}$ & $\begin{array}{l}\text { No risk (simpler design - and as flown on } \\
\text { MER) }\end{array}$ \\
\hline Smaller Gnomon & $\begin{array}{l}\text { Allowed by contraction of } \\
\text { total area of grayscale rings }\end{array}$ & Extremely low risk \\
\hline
\end{tabular}

principle a measure of the brightness ratio of sunlit to shadowed regions can be extracted from just the innermost ring, if necessary.

Table 1 summarizes all the changes from the heritage designs. Note that all of the changes represent low to no risk.

\subsubsection{Embellishments}

The Mastcam-Z primary calibration target contains a number of "embellishments" developed by the Mastcam-Z team in collaboration with the Planetary Society and approved by NASA. These are elements that do not have a direct engineering or scientific purpose but are added to the target for decoration and to assist efforts in outreach and public communication. This calibration target will become one of the most imaged objects on Mars and represents an opportunity for communicating the excitement of Mars exploration and of the Perseverance rover mission to the public. The embellishments are laser-engraved into the surface of the calibration target and consist of a motto, a set of graphics, and a short inspirational message to future Mars explorers that may happen on the rover someday. Motto and graphics are engraved on the top surface of the calibration target and will be visible to the Mastcam-Z. The message is engraved around the edge and largely invisible to the Mastcam-Z, but has been imaged pre-flight and will of course be visible on spare units that may be put on display at museums etc. For more details on these embellishments, see Kinch et al. (2020).

\subsection{Secondary Target}

The Mastcam-Z secondary calibration target is smaller and much simpler than the primary target and the design does not have the same flight heritage.

\subsubsection{Design Overview}

The secondary calibration target (Figs. 2-4) is a straight-forward angled "shelf" frame holding a total of 14 optical patches. Each optical patch is a square $10 \mathrm{~mm}$ by $10 \mathrm{~mm}$ and $2 \mathrm{~mm}$ thick. Four distinct grayscales and three distinct colors are each repeated twice, once on a vertical surface and once on a horizontal surface. The frame is bead-blasted aluminum and 

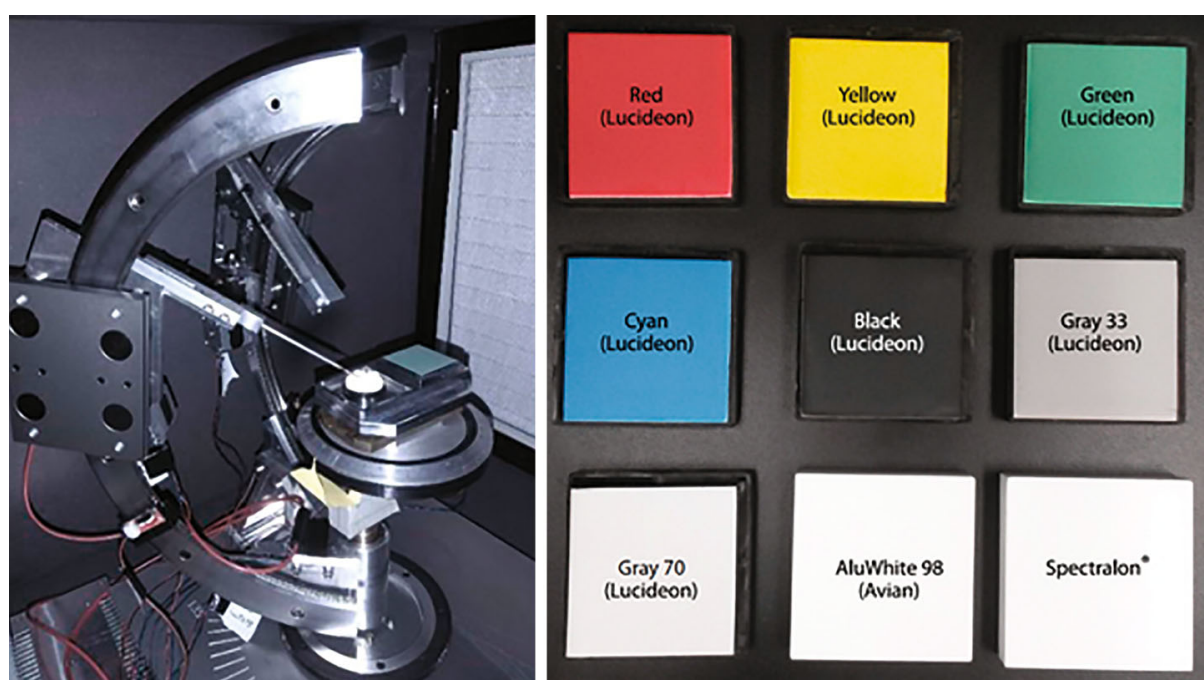

Fig. 5 Goniometer setup at the Niels Bohr Institute (left), witness samples (right). Right figure reproduced from Buz et al. (2019)

optical patches are fastened to the aluminum frame using a space-qualified epoxy adhesive. The colors and grayscales are identical to colors and grayscales on the primary target except the yellow is missing. The overall design is significantly simpler than the primary target with no embedded magnets and no gnomon. The mass is $15 \mathrm{~g}$ (compare with $103 \mathrm{~g}$ for the primary target).

The target fastens to a vertical surface using 4 fastener holes in a rectangular pattern with $44 \mathrm{~mm}$ horizontal distance and $23 \mathrm{~mm}$ vertical distance. The target measures $80 \mathrm{~mm} \times 30$ $\mathrm{mm} \times 16 \mathrm{~mm}$ (outside dimensions).

\section{Characterization}

This section describes a number of optical characterization experiments performed on assembled targets and on witness samples of the eight color and grayscale calibration target materials. Reflectance measurements were performed on the flight targets and witness samples at the Niels Bohr Institute, University of Copenhagen. Goniometric reflectance measurements were performed on witness samples at the California Institute of Technology and at the University of Bern. At the University of Winnipeg, a UV exposure experiment was conducted on samples of calibration target color and grayscale materials with reflectance spectra acquired before, during, and after completion of the UV illumination program. Finally, a "calibration spare" set of targets were imaged by the flight Mastcam-Z cameras during standalone instrument calibration at Arizona State University in May 2019 and the flight targets were imaged on the rover by the flight cameras during Assembly, Test and Launch Operations (ATLO) in October of 2019 at NASA JPL.

\subsection{Reflectance Spectra on Assembled Targets 400 nm - 1100 nm}

Figure 5 shows the goniometric reflectance spectrometer setup at the Niels Bohr Institute. Two automatically-controlled arms hold optical fibers. One fiber illuminates the sample with 


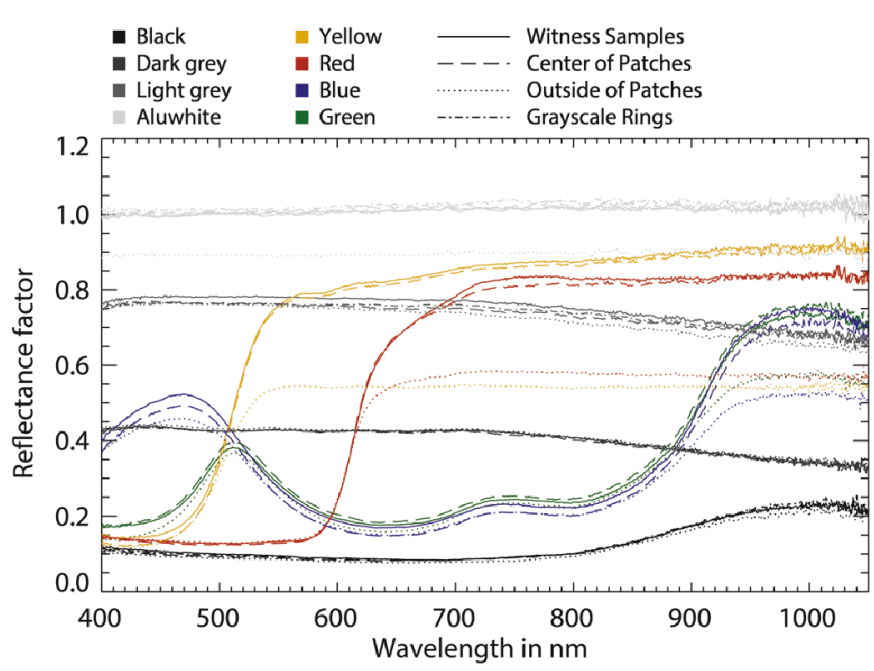

Fig. 6 Reflectance measurements on assembled flight primary target compared with witness samples. Performed at the Niels Bohr Institute at $\mathrm{e}=58^{\circ}, \mathrm{i}=0^{\circ}$

light from a halogen-lamp while another collects reflected light and conducts it to a spectrometer (AvaSpec 2048). The entire setup is enclosed in a black box to minimize stray light.

The measurement geometry is defined by three angles: The angle of incidence (i) is the angle between the direction from the target to the light source and the target surface normal. The angle of emission (e) is the angle between the direction from the target to the detector and the target surface normal. The azimuth angle is the angle between the plane of incidence and the plane of emission. When $\mathrm{e}=0^{\circ}$ and/or $\mathrm{i}=0^{\circ}$ the geometry is unchanged when azimuth is changed in the measurement apparatus. Reflectance measurements were conducted at $\mathrm{i}=0^{\circ}, \mathrm{e}=58^{\circ}$ (same emission angle at which Mastcam-Z will observe the primary calibration target). Measurements were conducted on assembled targets and on a set of $5 \mathrm{~cm} \times 5 \mathrm{~cm}$ witness samples (also shown in Fig. 5).

Figure 6 shows reflectance spectra measured on the flight primary calibration target as well as on the set of witness samples. In this section we will consistently present reflectance as "Reflectance factor" (or $\mathrm{R}^{*}$ ) defined as the reflectance relative to the reflectance of a perfect Lambertian scatterer illuminated and observed in the same geometry. The reflectance factor can be written as:

$$
R^{*}=R_{B D} \frac{\pi}{\cos (i)}=\frac{I}{F} \frac{1}{\cos (i)},
$$

where $\mathrm{R}_{\mathrm{BD}}$ is the bidirectional reflectance and $\mathrm{i}$ is the angle of incidence and $(\mathrm{I} / \mathrm{F})$ or "radiance factor" is the quantity presented in reflectance-calibrated Mastcam- $\mathrm{Z}$ images.

Spectra from witness samples match spectra from the center of color and grayscale patches and match spectra acquired on grayscale rings well (within the uncertainty of the measurement system primarily controlled by the stability of the power supply to the lamp). This agreement between witness samples and assembled targets allows for ongoing characterization of the witness samples to be employed to model the behavior of the flight target.

The outer part of the color and grayscale patches is very thin and for some patches partially transparent. This transparency is apparent (dotted lines) throughout the wavelength range for the white patch, for the yellow patch at all wavelengths above $\sim 500 \mathrm{~nm}$, for the 
Table 2 Reflectance factors for the 8 calibration target materials averaged over each Mastcam-Z band. Based on spectra acquired on witness samples at the Niels Bohr Institute at $\mathrm{i}=0^{\circ}, \mathrm{e}=58^{\circ}, \mathrm{az}=0^{\circ}$ and shown in Fig. 6 (solid lines)

\begin{tabular}{lllllllll}
\hline & White & Light gray & Dark gray & Black & Blue & Green & Yellow & Red \\
\hline L0B $(471 \mathrm{~nm})$ & 1.01 & 0.79 & 0.43 & 0.10 & 0.48 & 0.27 & 0.28 & 0.14 \\
L0G $(546 \mathrm{~nm})$ & 1.02 & 0.79 & 0.43 & 0.10 & 0.32 & 0.29 & 0.65 & 0.15 \\
L0R $(638 \mathrm{~nm})$ & 1.02 & 0.79 & 0.43 & 0.09 & 0.19 & 0.19 & 0.82 & 0.54 \\
R0B $(471 \mathrm{~nm})$ & 1.01 & 0.79 & 0.43 & 0.10 & 0.48 & 0.27 & 0.28 & 0.14 \\
R0G $(546 \mathrm{~nm})$ & 1.02 & 0.79 & 0.43 & 0.10 & 0.32 & 0.29 & 0.65 & 0.15 \\
R0R $(638 \mathrm{~nm})$ & 1.02 & 0.79 & 0.43 & 0.09 & 0.19 & 0.19 & 0.82 & 0.54 \\
L6 $(441 \mathrm{~nm})$ & 1.00 & 0.78 & 0.44 & 0.11 & 0.50 & 0.19 & 0.15 & 0.14 \\
L5 $(529 \mathrm{~nm})$ & 1.01 & 0.79 & 0.43 & 0.10 & 0.36 & 0.36 & 0.66 & 0.13 \\
L4 $(605 \mathrm{~nm})$ & 1.02 & 0.79 & 0.43 & 0.09 & 0.19 & 0.19 & 0.82 & 0.30 \\
L3 $(687 \mathrm{~nm})$ & 1.03 & 0.78 & 0.43 & 0.08 & 0.18 & 0.19 & 0.85 & 0.75 \\
L2 $(754 \mathrm{~nm})$ & 1.03 & 0.77 & 0.42 & 0.09 & 0.23 & 0.25 & 0.88 & 0.84 \\
L1 $(801 \mathrm{~nm})$ & 1.03 & 0.76 & 0.41 & 0.10 & 0.23 & 0.24 & 0.89 & 0.84 \\
R1 $(801 \mathrm{~nm})$ & 1.03 & 0.76 & 0.41 & 0.10 & 0.23 & 0.24 & 0.89 & 0.84 \\
R2 $(866 \mathrm{~nm})$ & 1.03 & 0.74 & 0.39 & 0.15 & 0.32 & 0.34 & 0.90 & 0.84 \\
R3 $(910 \mathrm{~nm})$ & 1.03 & 0.73 & 0.38 & 0.19 & 0.52 & 0.52 & 0.91 & 0.84 \\
R4 $(940 \mathrm{~nm})$ & 1.03 & 0.72 & 0.37 & 0.21 & 0.67 & 0.66 & 0.92 & 0.85 \\
R5 $(979 \mathrm{~nm})$ & 1.03 & 0.70 & 0.35 & 0.23 & 0.75 & 0.73 & 0.93 & 0.85 \\
R6 $(1012 \mathrm{~nm})$ & 1.03 & 0.69 & 0.35 & 0.23 & 0.73 & 0.73 & 0.92 & 0.84 \\
\hline
\end{tabular}

red patch above $\sim 600 \mathrm{~nm}$ and for the blue and green patches above $\sim 900 \mathrm{~nm}$, and indeed is visible in to the eye in Fig. 2 as well (yellow and red patches). These thin surfaces are there to control the positioning of the center of the patch relative to the underlying magnet and are not intended to be used as optical references (indeed, after a few sols on Mars, they will be covered by thick layers of dust attracted by the underlying magnets), so the spectral discrepancy is not a concern.

In Table 2, we have tabulated reflectance factors of the 8 witness samples averaged over the 18 Mastcam- $Z$ bands at $i=0^{\circ}, e=58^{\circ}, a z=0^{\circ}$.

\subsection{Goniometric Reflectance}

\subsubsection{Spectral Goniometry on Witness Samples $400 \mathrm{~nm}-2500 \mathrm{~nm}$}

A study of the scattering behavior of the 8 witness samples as a function of illumination geometry was conducted at the California Institute of Technology in 2018. Spectra from 400 $\mathrm{nm}-2500 \mathrm{~nm}$ were acquired in 76 geometries per sample. The main benefit of this dataset is the broad spectral coverage covering also wavelengths $>1000 \mathrm{~nm}$ relevant to the passive sensor of the SuperCam instrument on the Perseverance Rover (Wiens et al. 2020, this issue; Maurice et al. 2020, this issue).

This study has been separately published (Buz et al. 2019) and will only be briefly summarized here. The Buz et al. study generally concluded that the brighter samples (white, light gray, red and yellow at $800 \mathrm{~nm}$ ) show quasi-isotropic behavior. The white (AluWhite) sample is weakly backward scattering while the light gray, red, and yellow samples are 
Table 3 Bandpasses used for goniometric reflectance measurements with the PHIRE-2 instrument

\begin{tabular}{ll}
\hline Band center & Band FWHM \\
\hline $450 \mathrm{~nm}$ & $70 \mathrm{~nm}$ \\
$550 \mathrm{~nm}$ & $70 \mathrm{~nm}$ \\
$650 \mathrm{~nm}$ & $70 \mathrm{~nm}$ \\
$750 \mathrm{~nm}$ & $70 \mathrm{~nm}$ \\
$905 \mathrm{~nm}$ & $25 \mathrm{~nm}$ \\
$1064 \mathrm{~nm}$ & $25 \mathrm{~nm}$
\end{tabular}

weakly forward scattering. The darker samples (black, dark gray, blue and green at 800 $\mathrm{nm}$ ) show significant forward-scattering behavior. Generally, the photometric properties of the samples vary with illumination in consistent ways that can be described. Importantly, however, the magnitude of the photometric effect in each sample is found to be wavelengthdependent, thereby necessitating different corrections for viewing geometry as a function of wavelength so as to obtain accurate band depths and spectral slopes. None of the samples show any significant specular effect.

\subsubsection{Bandpass Goniometric Reflectance}

An extensive set of bandpass goniometric reflectance measurements were conducted on the 8 witness samples using the Physikalisches Institut Radiometer Experiment 2 (PHIRE-2) goniometer-reflectometer instrument at the Planetary Ice Laboratory at the University of Bern. The instrument is described in detail in Pommerol et al. (2011). The light source is a 250W Quartz Tungsten Halogen Lamp with an active feedback from a control detector to the lamp power supply to ensure a very high stability. The light is conducted through an optical fiber to the end of a $60 \mathrm{~cm}$ arm that can be elevated over the full range from horizontal to vertical. The detector (NewportCorp. MerlinTM \#70104) is located on a $50 \mathrm{~cm}$ arm that can also be elevated over the full range from horizontal to vertical as well as traversed over one half circle in azimuth.

Measurements were conducted through 6 bandpass filters listed in Table 3. For each of the 8 witness samples, a total of 7824 measurements were conducted: 1304 measurements per band, stepping through emission angles from 0 to 80 degrees in steps of $5^{\circ}$ and through azimuth from $0^{\circ}-180^{\circ}$ in steps of $15^{\circ}$ for 6 values of the angle of incidence $\left(0^{\circ}, 15^{\circ}, 30^{\circ}\right.$, $45^{\circ}, 58^{\circ}$ and $70^{\circ}$ ). Measurements were conducted at room temperature.

The principle of reciprocity states that the reflectance factor is unchanged when the angles of incidence and emission are swapped. The PHIRE-2 instrument is designed for easier actuation of the detector arm than the light source arm. Therefore, we mapped in smaller steps in angle of emission than in angle of incidence, although during operations on Mars the angle of emission will be fixed by the position of the calibration targets relative to the Mastcam-Z, whereas the angle of incidence will change with the position of the Sun and with rover tilt and orientation. During the measurement program it was confirmed that the instrument obeys the principle of reciprocity to within $5 \%$ for all samples and all bands. We can therefore employ this principle when using this dataset, and, in particular, use the set of measurements at $\mathrm{i}=58^{\circ}$ to model reflectance factors of the calibration target on Mars at $\mathrm{e}=$ $58^{\circ}$, which is the angle of emission from the surface of the primary Mastcam-Z calibration target towards the Mastcam- $Z$ camera.

Figure 7 shows measurements on the 8 witness samples stepping the detector arm through the principal plane at $i=0^{\circ}$. For all samples the reflectance factor drops at high emission an- 


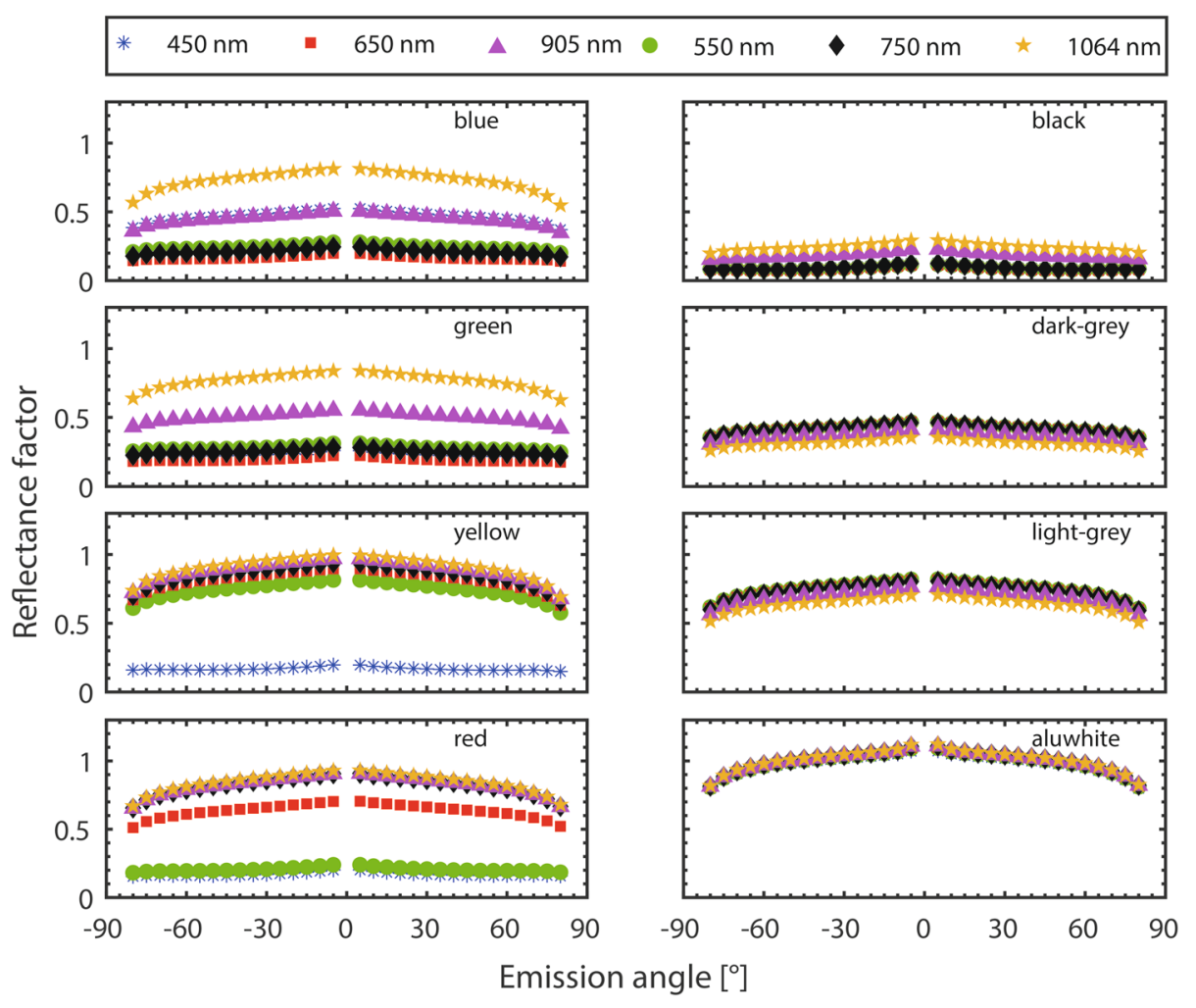

Fig. 7 Goniometric reflectance measurements using the PHIRE-2 instrument on the set of witness samples stepping the detector arm through the principal plane at $\mathrm{i}=0^{\circ}$. Here negative emission angles are used as shorthand for Azimuth $=180^{\circ}$, positive angles are Azimuth $=0^{\circ}$

gles. The closest we get to opposition in these measurements is e $=5^{\circ}$ at $\mathrm{i}=0^{\circ}$. At that resolution none of the samples show a pronounced opposition peak, although the AluWhite98 sample does show a bit more than the other samples. It was not a priority to map out the opposition peak at high resolution since on Mars the calibration target will be shadowed by the mast when we get close to opposition.

Figure 8 shows measurements stepping the detector arm through the principal plane at $\mathrm{i}=58^{\circ}$ degrees. By the principle of reciprocity this is equivalent to stepping the illumination through the principal plane at $\mathrm{e}=58^{\circ}$, which is relevant for the geometry we will experience on Mars. The AluWhite 98 sample shows a quite flat profile at all wavelengths for e $<45^{\circ}$, so on Mars this material can be expected to have relatively uniform reflectance anytime the sun is more than $45^{\circ}$ above the horizon - which will be the case for the majority of observations, since the landing site at Jezero Crater is at 18.38 degrees north and the rover will mostly be active around noon. At low sun in a forward-scattering geometry the reflectance increases slightly. All the other (Lucideon) samples show a significantly forward-scattering tendency, particularly the darker samples. The reflectance curve is flat in the backscattering geometry and also relatively flat for low angles of emission $\left(<45^{\circ}\right)$ in the forward-scattering geometry (or equivalently, on Mars, for high sun in the forward-scattering geometry).

Figure 9 shows the full set of measurements at $i=58^{\circ}$ for one wavelength band $(650$ $\mathrm{nm})$. The behavior is as described based on the principal-plane data in Fig. 8: relatively 


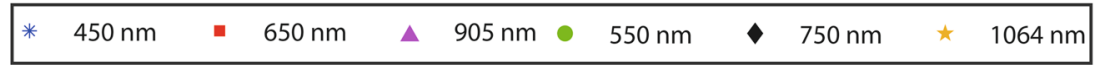
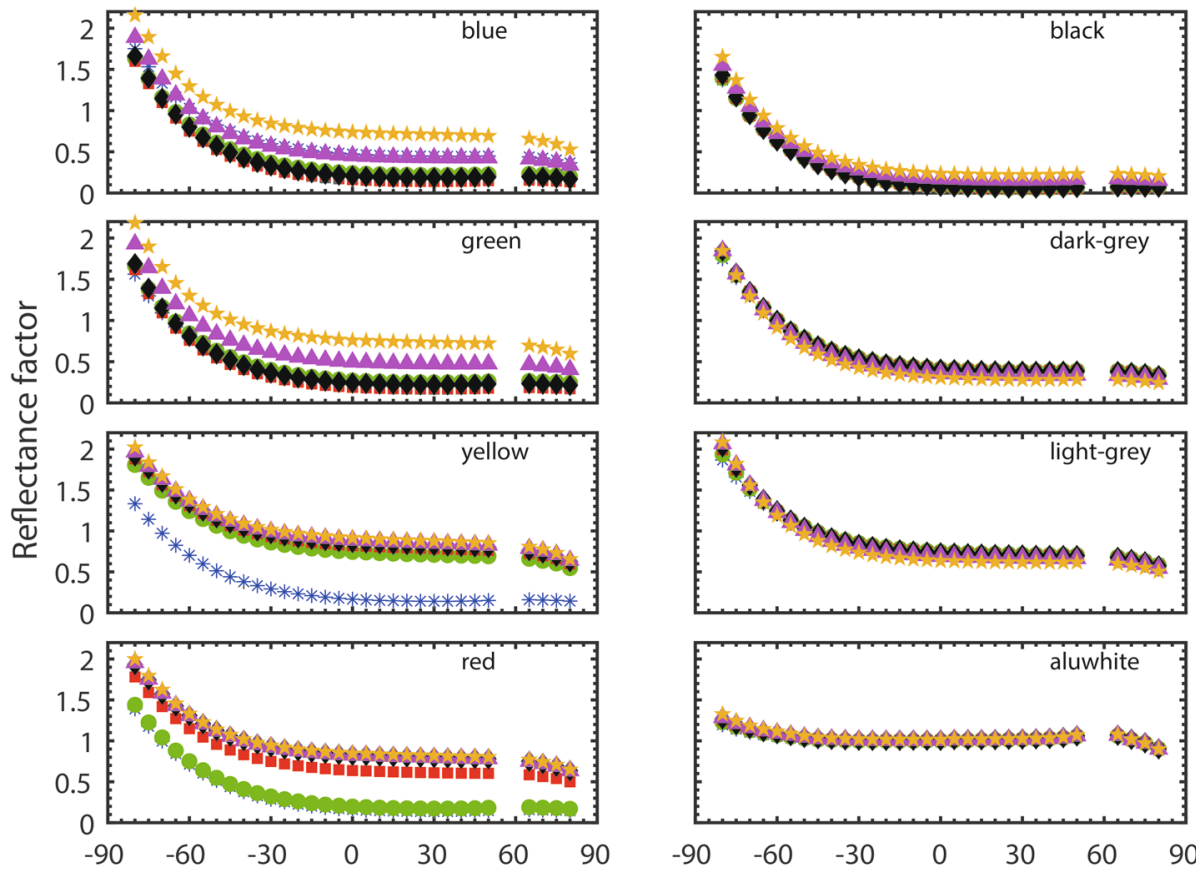

Emission angle $\left[^{\circ}\right]$

Fig. 8 Goniometric reflectance measurements using the PHIRE-2 instrument on the set of witness samples stepping the detector arm through the principal plane at $\mathrm{i}=58^{\circ}$. Here negative emission angles are used as shorthand for Azimuth $=180^{\circ}$, positive angles are Azimuth $=0^{\circ}$. By the principle of reciprocity this is equivalent to varying Solar position at a fixed $\mathrm{e}=58^{\circ}$ as will be the case on Mars

flat for AluWhite98, dominated by a significant forward-scattering peak for high emission angles.

The behavior observed in the bandpass goniometric dataset from the PHIRE-2 instrument is qualitatively consistent with what was observed in the study by Buz et al. (2019) (see also Sect. 4.2.1). More quantitative comparison studies will be conducted ahead of landing on Mars. Based on the set of data from the Niels Bohr Institute (4.1), from Caltech (4.2.1), from Bern (4.2.2) and measurements underway at Western Washington University we will produce a model to accurately predict reflectance factor at any illumination geometry, for all calibration target materials and at all Mastcam-Z filters. Operationally, this model will be augmented by a dust correction algorithm as discussed in Sect. 5.5.

\subsection{UV Illumination}

An ultraviolet (UV) exposure study was conducted at the Centre for Terrestrial and Planetary Exploration, University of Winnipeg, in the late fall of 2019. Here, samples of the 8 color and grayscale materials were exposed to a Mars surface-like UV irradiance while in a Marslike atmosphere.

The Mini-ME vacuum chamber was used to simulate Mars surface conditions (e.g., Craig et al. 2001). Sample temperature was maintained at $\sim 10{ }^{\circ} \mathrm{C}$ by circulating a $1{ }^{\circ} \mathrm{C}$ 

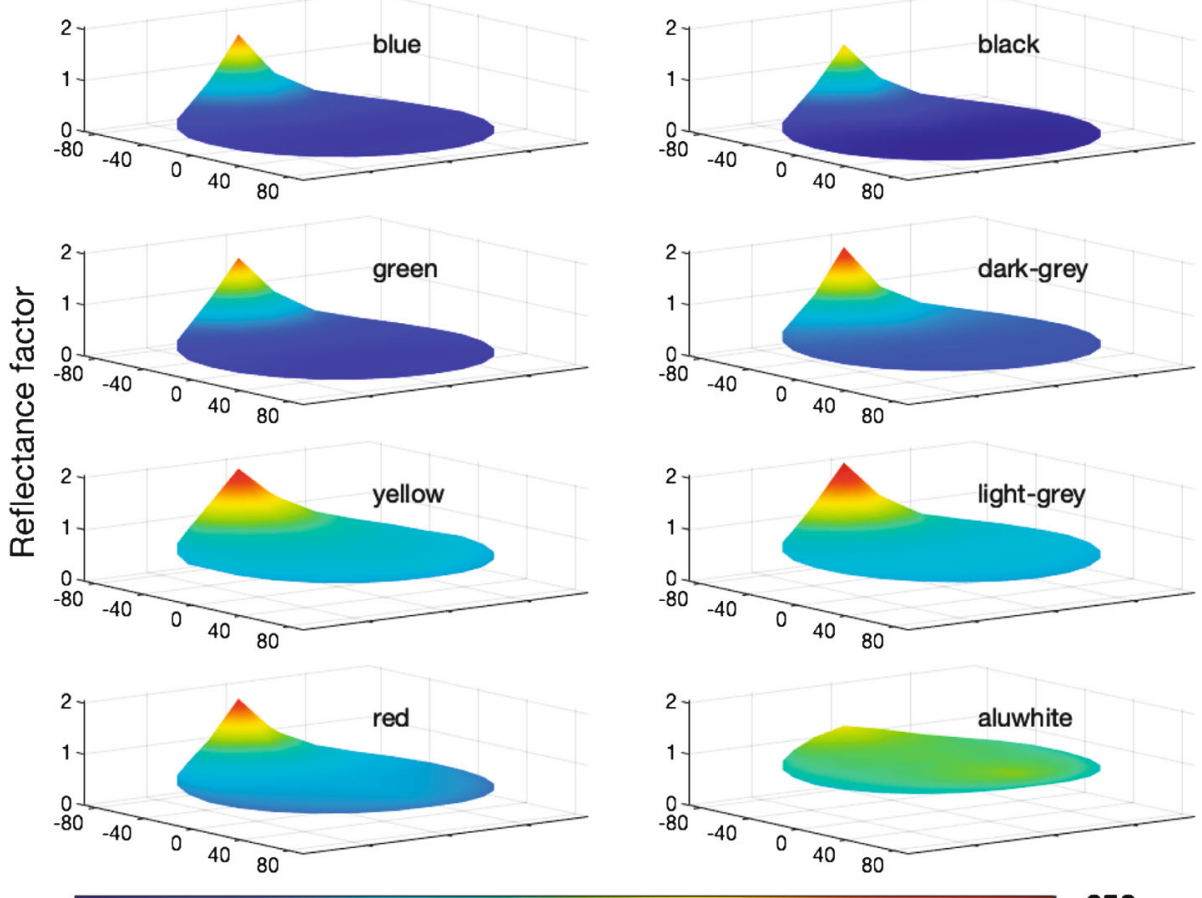

0.5

1.5

2

Fig. 9 Goniometric reflectance measurements using the PHIRE-2 instrument on the set of witness samples stepping the detector arm through a full hemisphere at $\mathrm{i}=58^{\circ}$. Shown are measurements for $650 \mathrm{~nm}$. Here negative emission angles are used as shorthand for azimuth $=180^{\circ}$, positive angles are azimuth $=0^{\circ}$. The second hemisphere is filled in by mirroring in the principal plane. By the principle of reciprocity this is equivalent to varying Solar position at a fixed $\mathrm{e}=58^{\circ}$ as will be the case on Mars

liquid through an aluminum plate in contact with the samples inside the vacuum chamber; a commercial-grade, dry $\mathrm{CO}_{2}$ atmosphere was maintained by allowing a constant flow through the chamber, unless otherwise noted below.

The samples were initially exposed to a $\sim 100 \mathrm{~Pa} \mathrm{CO}_{2}$ atmosphere and $\sim 10{ }^{\circ} \mathrm{C}$ surface temperature for 4 days. The vacuum chamber was sealed, and no outgassing was observed at $1 \mathrm{~Pa}$ intervals. Following this, the samples were exposed to a $650 \mathrm{~Pa} \mathrm{CO}_{2}$ atmosphere at $\sim 10{ }^{\circ} \mathrm{C}$ with a stepwise dose of UV. At the end of each step, the samples were removed from the vacuum chamber and reflectance spectra were acquired in air.

Reflectance spectra over the 350- to 2500-nm interval were measured with an Analytical Spectral Devices LabSpec 4 Hi Res spectrometer with a spectral resolution of between 3 and $6 \mathrm{~nm}$ and spectral sampling interval of $1.4 \mathrm{~nm}$ for the 350 to $1000 \mathrm{~nm}$ interval and $2 \mathrm{~nm}$ for the 1000 - to $2500-\mathrm{nm}$ interval. The data are internally resampled by the instrument to output data ultimately at 1-nm intervals with a cubic spline interpolation. Spectra were measured at a viewing geometry of $i=30^{\circ}$ and $e=0^{\circ}$ with incident light being provided by an inhouse quartz-tungsten-halogen collimated light source $\left(<1.5^{\circ}\right.$ divergence $)$. Sample spectra were measured relative to a Spectralon ${ }^{\circledR} 99 \%$ diffuse reflectance standard and corrected for minor (less than $\sim 2 \%$ ) irregularities in its reflectance. For the spectral data acquisition 500 spectra of the dark current, standard, and sample were acquired and averaged, to provide high signal-to-noise 

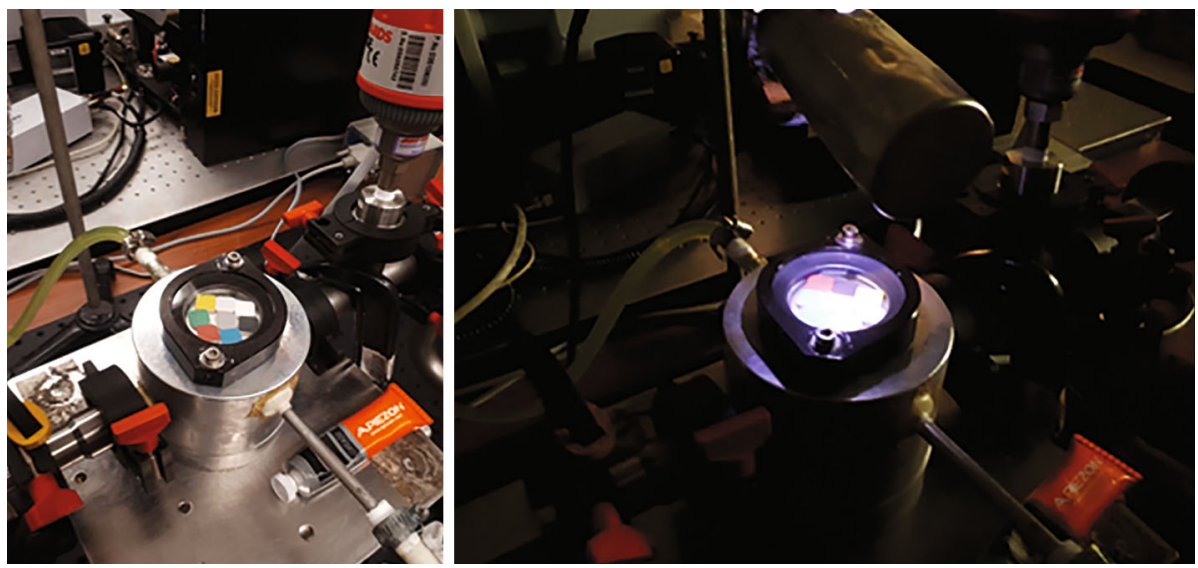

Fig. 10 Experimental setup for UV-exposure experiments. Left: UV-lamp off, Right: UV-lamp on

The Mars surface equivalent doses were quantified. A Heraeus DO802 deuterium lamp was used, and the spectral radiance was assumed to be identical to that of the DO902 measured by Hollandt et al. (2000), which has near-identical specifications. Figure 10 shows the experimental setup with and without the DO802 bulb turned on. A solarmeter 8.0 UVC radiometer was used to quantify the irradiance of the DO802 at the same placement of the samples in the chamber; $50 \mathrm{~mm}$ from the DO802 aperture and through the $9.4 \mathrm{~mm}$ sapphire window, with a resulting value of $0.25 \mathrm{~W} \mathrm{~m}^{-2}$. The radiance spectra of the DO802 were normalized to the measured irradiance at $258 \mathrm{~nm}$, then multiplied by the transmittance of the sapphire window (normalized to unity at $258 \mathrm{~nm}$ ) which was measured from 180 to 6000 $\mathrm{nm}$. Dual-pass transmittance off of a halon reflectance standard inside the vacuum chamber showed that there was no significant difference in atmospheric absorption (180-2500 $\mathrm{nm}$ ) between air and $400 \mathrm{~Pa} \mathrm{CO}_{2}$, likely due to the path length of only 1-2 mm through atmosphere inside the chamber. As such, any atmospheric absorption was ignored.

The resulting experimental irradiance spectra were compared to two extreme UV irradiance scenarios at the surface of Mars: 1 . during northern summer $\left(\mathrm{Ls}=70^{\circ}\right)$ for low dust loading ( $\tau=0.1$ ), at the equator and local noon (taken from Patel et al. 2002), and 2. during spring (vernal equinox) for a dusty day $(\tau=2.0)$, at $60^{\circ} \mathrm{N}$ and local noon (taken from Cockell et al. 2000). Both scenarios were assumed to scale linearly to an irradiance of $0 \mathrm{~W} \mathrm{~m}^{-2}$ at $180 \mathrm{~nm}$ from their data cutoff wavelengths (190 and $195 \mathrm{~nm}$, respectively).

The resulting irradiance ratios (Fig. 11) were weighted for photon energy, since bonding dissociation and spectral alteration increase with photon energy. The spectra are multiplied by their photon energy after the energy spectrum is normalized to unity at $290 \mathrm{~nm}$, which is the crossover point where experimental irradiance equals scenario 1. One experimental day was found to be roughly equivalent to 3.4 martian years of solar exposure for scenario 1 , and roughly 260 martian years for scenario 2 . The mean of the two scenarios, 132 martian years, was used to quantify the experimental exposure.

Thus, reflectance spectra were acquired at simulated Mars surface sunlight exposures of $1,2,4,8,16,32,64$, and 128 months ( $~ 5$ martian years). Finally, a measurement was acquired at the equivalent of $\sim 100$ martian years UV exposure on the Martian surface.

Figure 12 shows that for the white sample (Avian AluWhite98), significant spectral reddening was observed over the 350 to $1000 \mathrm{~nm}$ range. At $450 \mathrm{~nm}$ the reflectance was reduced by about $2.5 \%$ with 32 -months-equivalent irradiation, while above $700 \mathrm{~nm}$ the observed 


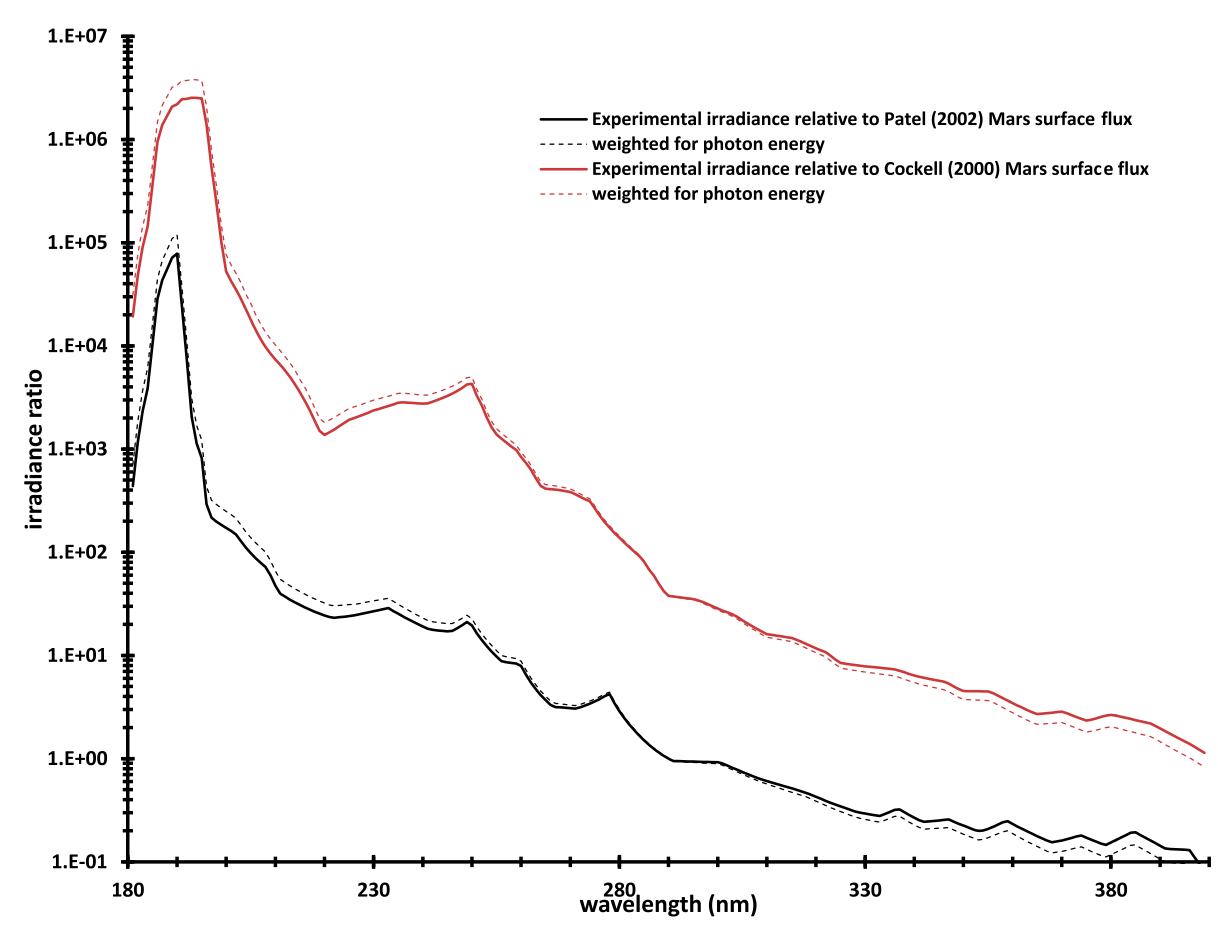

Fig. 11 Quantified relative irradiance of the UV experiment compared to two scenarios at the surface of Mars (see Sect. 4.3 text for scenarios). Ratio of lamp irradiance $\left(\mathrm{W} /\left(\mathrm{m}^{2} \mathrm{~nm}\right)\right)$ versus the published scenario

change was a small fraction of a percent. The spectral changes also occurred during the vacuum-only step, and are therefore not entirely dependent on UV irradiance. Limited spectral changes occurred during the first simulated martian year. For all Lucideon samples (e.g., Fig. 13) zero to only very limited spectral alteration was observed after $\sim 100$ years equivalent of UV-irradiance.

We conclude that the UV flux at the surface of Mars will have minimal effect on the use of the Mastcam-Z calibration targets. What effects that do occur are largely constrained to the white patch and ring and can be monitored by comparing to the other color and grayscale standards.

\subsection{Pre-Flight Imaging of Calibration Targets Using Flight Cameras}

Several sets of images of the Mastcam-Z calibration targets were acquired during pre-flight instrument calibration activities. During standalone instrument calibration at Arizona State University in May of 2019 more than 500 images of the "calibration spare" (serial \#017) primary calibration target and the "engineering model" (serial \#002) secondary target were acquired, including a set of images of the primary target using the full Mastcam-Z filter set at high resolution (100 $\mathrm{mm}$ focal length). In addition, a long series of image acquisitions were acquired in both broadband filters varying the zoom settings in small steps across the full range.

During ATLO testing in October 2019 images were acquired of the calibration target mounted on the rover using the flight cameras also in position on the rover mast. Figure $3 \mathrm{a}$ ) 


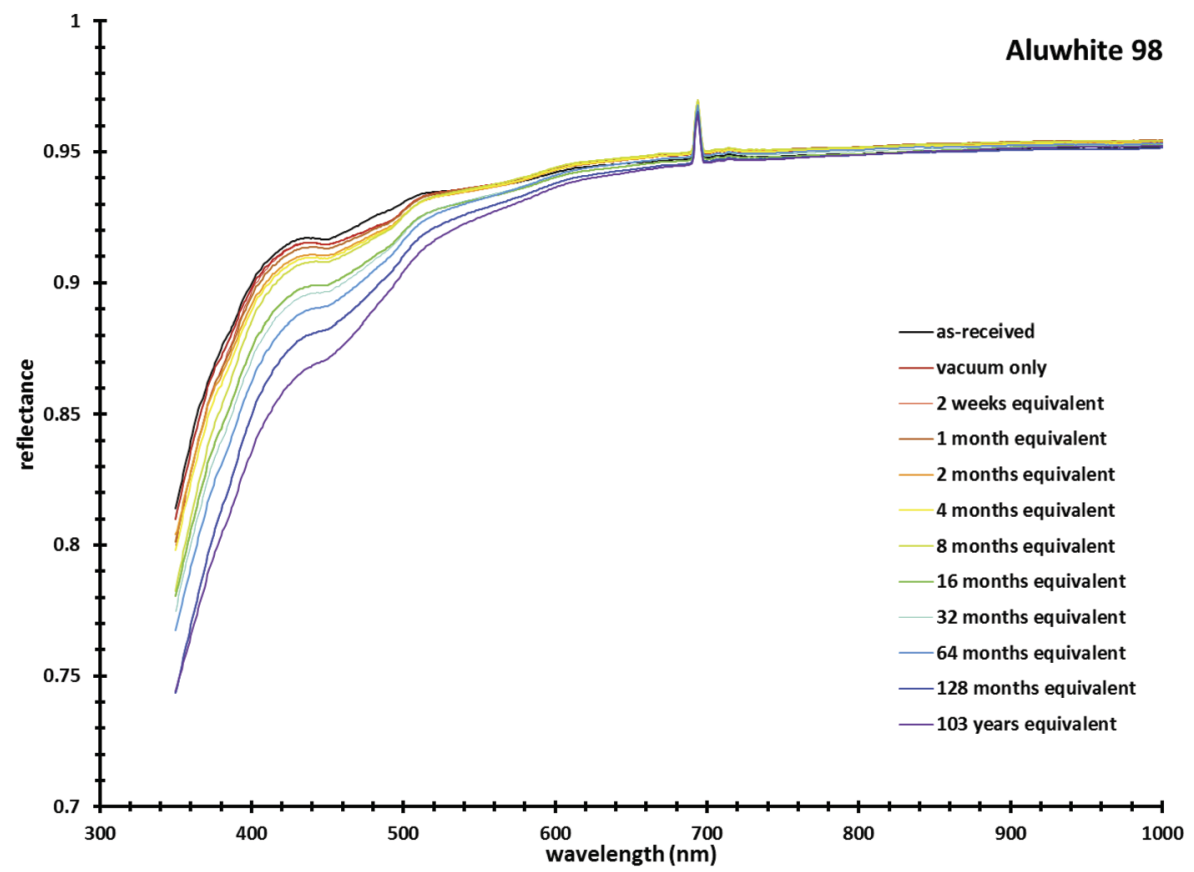

Fig. 12 UV-exposure experiments. Evolution of spectra of white sample (AluWhite98)

shows an image acquired in the broadband L0 filter at low resolution (27 $\mathrm{mm}$ focal length). Note the SuperCam calibration target also visible in the frame. A full filter set was acquired at the two ends of the resolution scale (110 $\mathrm{mm}$ focal length and $27 \mathrm{~mm}$ focal length) and a number of exposures were acquired in the broadband filters at intermediate zoom settings. This was repeated on the 26th and 29th of October 2019. Figures 14 and 15 show a comparison between images acquired in the L1 $(805 \mathrm{~nm})$ filter at two ends of the resolution scale (110 $\mathrm{mm}$ focal length and $27 \mathrm{~mm}$ focal length). It is apparent that at the lowest resolution there is significant pixelation which may complicate the markup and extraction of radiance values, in particular from the centers of the color and grayscale patches, where the dust-free area is expected to be only about $3 \mathrm{~mm}$ in diameter (see Sect. 5.2). The series of images acquired at varying resolution both during standalone calibration and ATLO will be used to determine the ideal zoom setting for calibration target imaging during flight, which will be a trade-off of data volume versus resolution versus compression.

Figure 16 shows a comparison of spectra acquired of the four grayscale rings as well as the eight color and grayscale patches using the Mastcam-Z during ATLO on October 26th to the spectra acquired on the witness samples (solid lines in Fig. 16). For each filter, the image data was fit to the laboratory spectra by a simple linear fit of the values derived from the 8 color and grayscale patches. The bias behavior of the Mastcam- $\mathrm{Z}$ is complex (see Hayes et al. 2021, Sect. 3.3.3) and work is ongoing to accurately model it. In the absence of zero-exposure frames acquired for this sequence we included an offset term in the linear fit, so we effectively perform bias subtraction by comparison to the calibration target. The resulting fit is quite satisfying and demonstrates the capability of the Mastcam-Z to measure reflectance spectra during flight. 


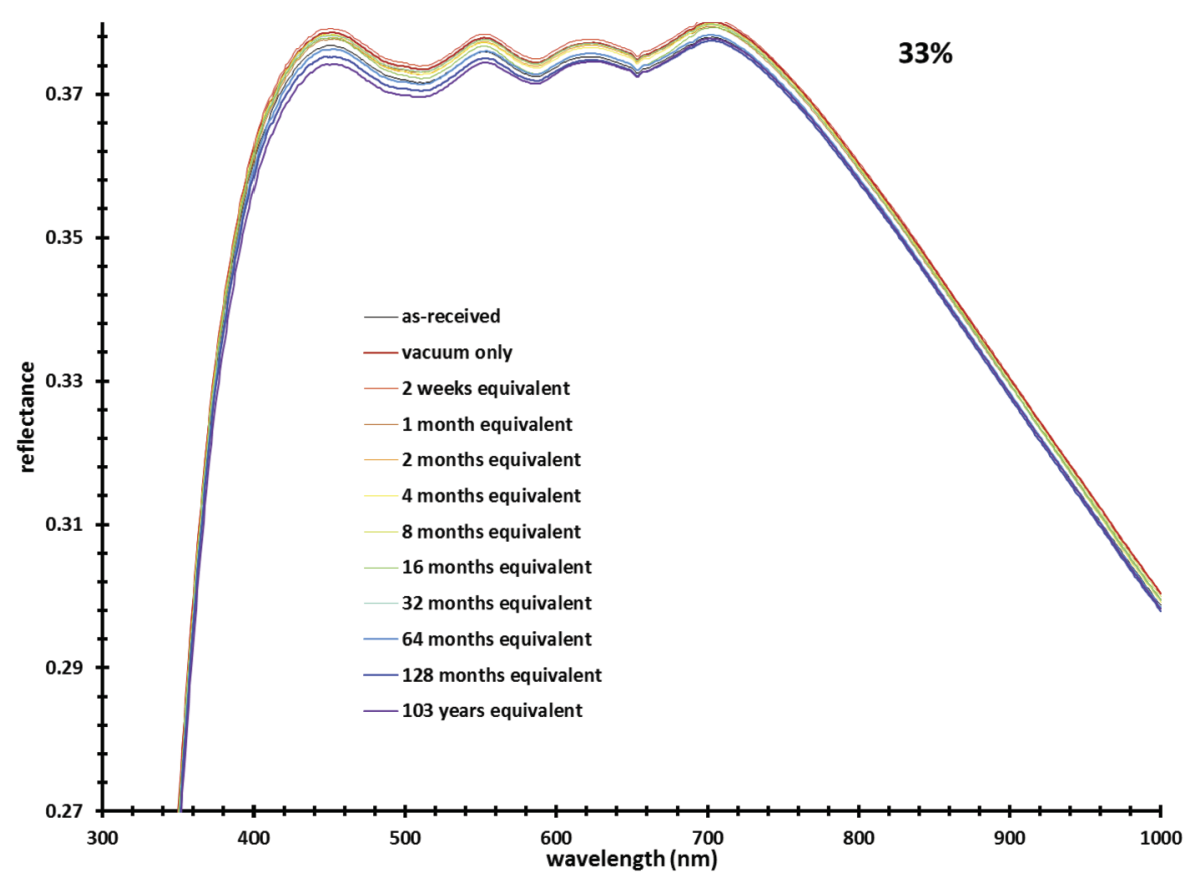

Fig. 13 UV-exposure experiments. Evolution of spectra of dark gray sample

\section{Operations}

\subsection{Position on Rover}

The Mastcam- $Z$ calibration targets are positioned on the rear starboard side of the Mars 2020 Perseverance rover deck. Figure 3 shows the targets in position, imaged by the flight cameras as well as a drawing of the rover with the location of the Mastcam- $Z$ calibration targets on the RPFA box pointed out. Table 4 lists values for distance and pointing from the camera head to the two calibration targets based on the rover CAD model.

\subsection{Effect of Primary-Target Embedded Magnets}

The effect of the magnetic field from the magnets on the trajectory of a magnetized dust grain is a complex interplay of gravity, drag, Brownian motion and magnetic forces (Kinch et al. 2006). Patterns of dust deposition are functions of wind speed, and dust grain size, density, and magnetic properties. As such there is no simple prescription for exactly how wide the "clean" spot in the center of the color and grayscale patches will turn out to be during operations on the Martian surface. Nonetheless, wind tunnel experiments, numerical simulation, and flight experiences from the MER, Phoenix and MSL missions provide useful indications.

This accumulated experience indicates that the larger Phoenix-type magnets that are part of the Mastcam-Z calibration target will deliver a central "clean" spot with low and uniform dust deposition of at least $3 \mathrm{~mm}$ radius. The equivalent minimum clean spot for the smaller MER-type magnets that flew on MER and MSL is $2 \mathrm{~mm}$ diameter. These numbers are illustrated by Figs. 17-20.

On the Mastcam-Z calibration targets we expect the "clean" spots to be circles at least 3 $\mathrm{mm}$ in diameter. Using $1.1 \mathrm{~m}$ as a camera range and using $3 \mathrm{~mm}$ as the low estimate of the 
Fig. 14 Flight Mastcam-Z primary calibration target mounted on the rover and imaged using flight cameras, L1 (805 $\mathrm{nm}$ ) filter at $110 \mathrm{~mm}$ focal length, compare Fig. 15

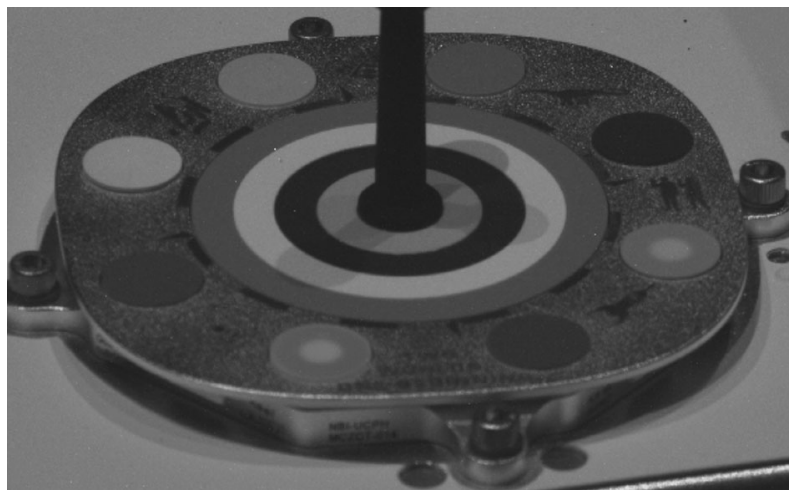

Fig. 15 Flight Mastcam-Z primary calibration target mounted on the rover and imaged using flight cameras, L1 (805 $\mathrm{nm}$ ) filter at $27 \mathrm{~mm}$ focal length, compare Fig. 14

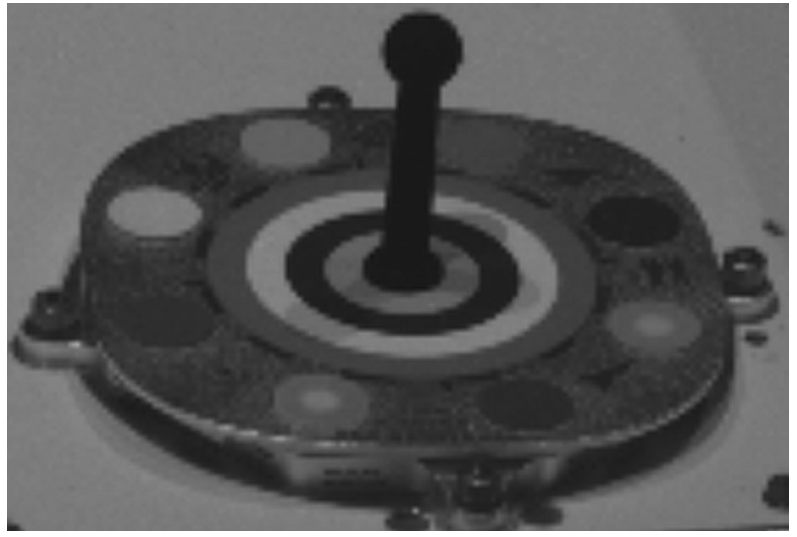

"clean" spot diameter, we can calculate the angular width of the "clean" spot. Clean spot width (azimuthal dimension) $=3 \mathrm{~mm} / 1.1(\mathrm{~m} / \mathrm{Rad})=2.73 \mathrm{mRad}$.

In the elevation dimension the angular width is smaller because we're viewing the cal target at an emission angle of $58^{\circ}$. Clean spot width (elevation dimension) $=2.73 \mathrm{mRad}$. $\cos \left(58^{\circ}\right)=2.73 \mathrm{mRad} \cdot 0.53=1.45 \mathrm{mRad}$.

So, viewed from Mastcam-Z the "clean" spots will appear as ellipses with (rough numbers) major axis $=2.73 \mathrm{mRad}$ and minor axis $=1.45 \mathrm{mRad}$.

The resolutions attainable by Mastcam-Z span the range between $0.28 \mathrm{mRad} / \mathrm{px}$ at $26 \mathrm{~mm}$ focal length (widest field) to $0.067 \mathrm{mRad} / \mathrm{px}$ at $111 \mathrm{~mm}$ (narrowest field). At $0.28 \mathrm{mRad} / \mathrm{px}$ the magnet clean spot will be roughly 10 by 5 pixels. At $0.067 \mathrm{mRad} / \mathrm{px}$ the magnet clean spot will be roughly 40 by 20 pixels. Note that these ranges are pixels on the detector. The Bayer patterns means that only $1 / 4$ of these will be blue pixels, $1 / 4$ red pixels and $1 / 2$ green pixels, so for broadband RGB filters as well as for many of the narrowband filters the number of active pixels is reduced accordingly (see Hayes et al. 2021). Operationally the calibration target will be imaged using a zoom setting that presents a reasonable compromise between number of pixels for data analysis and total data volume required. This sweet spot is not precisely known at present but will be somewhere between the two ranges given above (see Figs. 14 \& 15 and Sect. 4.4).

On MSL, the cal target is severely out of focus when viewed through the right eye of the Mastcam (M100). When viewed through the left eye (M34) the smaller MSL magnets have clean spots of about 7 by 4 pixels (Figs. $18 \& 19$ ). The Mastcam-Z system will thus be a 


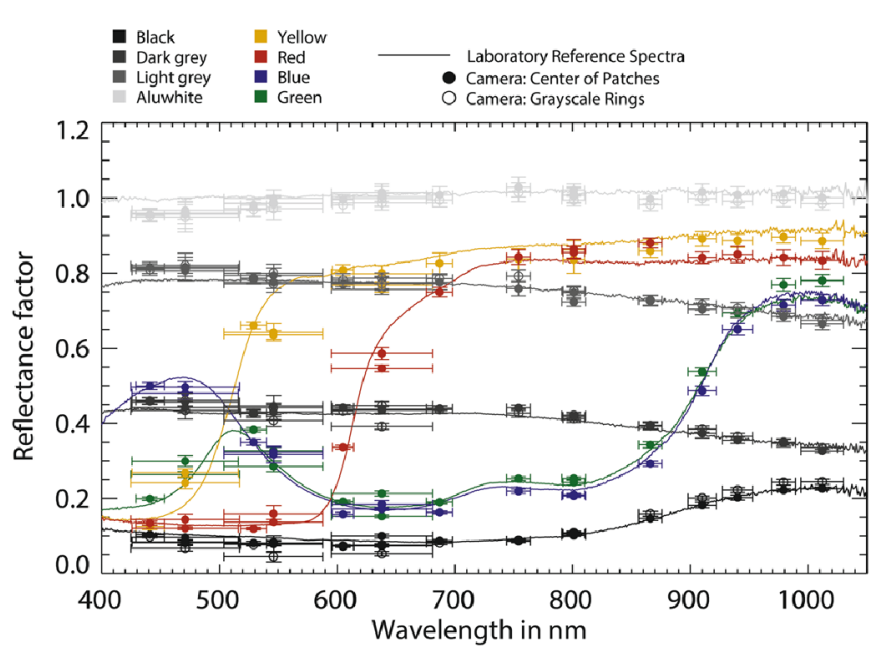

Fig. 16 Spectra derived from image sequence acquired at $27 \mathrm{~mm}$ focal length during ATLO imaging the flight primary calibration target using the flight camera. Spectra found by a linear two-term fit of image radiances derived from the 8 color and grayscale patches to laboratory spectra. Curves: Laboratory spectra on witness samples (solid lines from Fig. 21). Filled circles: Centers of color and grayscale patches. Open circles: Grayscale rings. Horizontal error bars are the width (FWHM) of the Mastcam-Z bands while vertical error bars are standard deviation between extracted DN values from pixels in regions of interest marked on the image

Table 4 Distances and pointings camera to calibration targets in rover navigation frame. Remote Sensing Mast elevation convention is $0^{\circ}$ when camera pointed horizontally and negative downwards from that. Emission angle for reflected light emitted from target towards camera is thus $90^{\circ}+\mathrm{RSM}$ elevation. Azimuth convention is $0^{\circ}$ when camera pointed straight ahead and positive counterclockwise from that. These numbers are based on the rover CAD model. Final numbers for the rover as built are not yet available at the time of writing and may deviate slightly

Mastcam-Z left eye

$1115 \mathrm{~mm}$

$-32.6^{\circ}$

$+165.7^{\circ}$

$-35.9^{\circ}$

$+165.0^{\circ}$

Mastcam-Z right eye

Distance front face of camera lens to primary target base of gnomon when camera pointed at target

Remote Sensing Mast elevation actuation when camera pointed to primary target base of gnomon

Remote Sensing Mast azimuth actuation when camera pointed to primary target base of gnomon

Remote Sensing Mast elevation actuation when camera pointed to secondary target center

Remote Sensing Mast azimuth actuation when camera pointed to secondary target center

$1116 \mathrm{~mm}$

$-32.1^{\circ}$

$+178.6^{\circ}$

$-35.3^{\circ}$

very significant improvement over MSL Mastcam in terms of number of pixels available in the clean spots.

As an additional point, it can be seen in Figs. $19 \& 20$ that even in the center of the magnet the radiance profile is not entirely flat indicating that even the "clean" area is not entirely free of dust. The larger magnets employed in the current design are expected to more efficiently repel dust and so keep the center of the clean area cleaner than was the case on MER/MSL. 


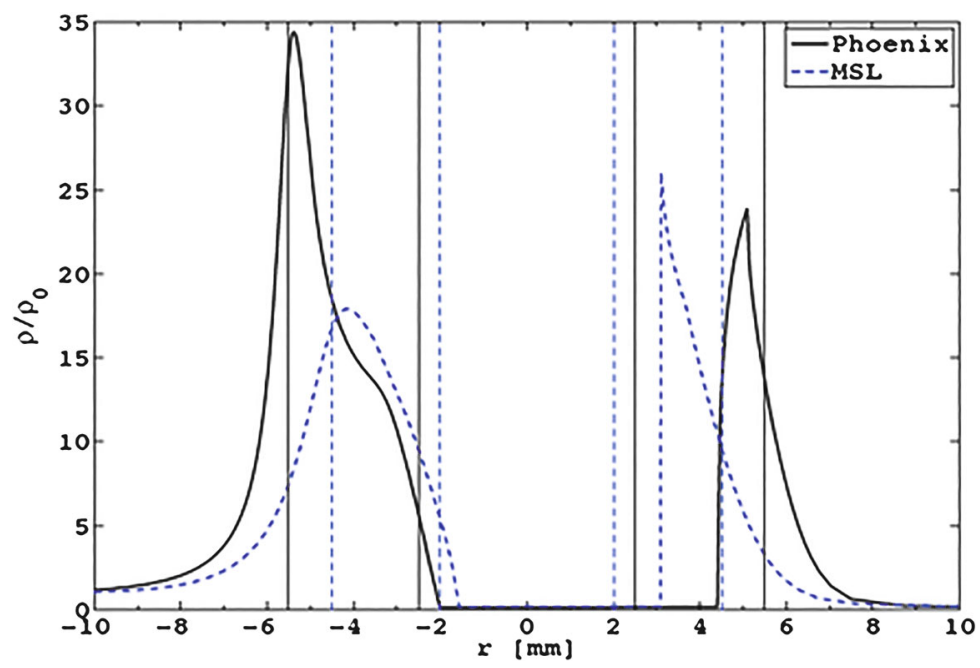

Fig. 17 Results from a 2D numerical simulation of dust capture on the larger M2020/Phoenix-type sweep magnets and the smaller MER/MSL style magnets. The curves show normalized density of settled dust where 1 is the settled dust density in the absence of magnetic fields. The vertical lines show the positions of the inner and outer edge of the magnets (underneath the surface). Simulation parameters: Friction velocity: 0.36 $\mathrm{m} \mathrm{s}^{-1}$ (equivalent to roughly 3-4 $\mathrm{m} \mathrm{s}^{-1}$ free-flow wind velocity). Dust grain diameter: 2 microns. Dust grain magnetization: $3.9 \mathrm{~A} \mathrm{~m}^{2} \mathrm{~s}^{-1}$. The wind direction is from the left. Dust on the upwind (left) side approaches closer to the center than dust on the downwind side. In a real-world situation, wind directions would vary and so the upwind (left) side of the profile is what would determine the size of the clean spot. With these parameters the clean spot is predicted to be almost $4 \mathrm{~mm}$ for the Phoenix case (dust density drops to 0 about $2 \mathrm{~mm}$ from the center on the upwind side) and about $3 \mathrm{~mm}$ for the MSL case (dust density drops to 0 about $1.5 \mathrm{~mm}$ from the center on the upwind side). Simulation by Lars von Moos

\subsection{In-Flight Imaging}

During Mars surface operations, the Mastcam-Z radiometric calibration targets will be regularly imaged. Typically, Mastcam- $Z$ will acquire images of the calibration targets within 30-60 minutes of the period when the most recent sequence of images was acquired, particularly if these images include multispectral observations (i.e., the narrow-band filters on each camera). Sub-frames of Mastcam-Z images that cover the calibration targets will be returned to Earth to assist with conversion of Mastcam-Z images to reflectance. The secondary target will in most cases be imaged in the same frame, but the secondary target image data will most likely not be used in the standard ground data reduction pipeline. Rather these images will be used intermittently to perform a manual check of results from the primarytarget analysis. As discussed in Sects. 4.4 and 5.2, an optimal value will be determined for the focal range to be employed when the calibration targets are imaged.

\subsection{Use of Calibration Targets for Reflectance Calibration}

\subsubsection{Data Reduction}

The Mastcam- $Z$ data reduction pipeline is described in detail in the work by Hayes et al. (2021). The raw images are converted through a series of steps (bias subtraction, dark current subtraction, flatfielding, etc.) into images calibrated to radiance $\left(\mathrm{W} /\left(\mathrm{m}^{2} \mathrm{~nm} \mathrm{sr}\right)\right.$. The 


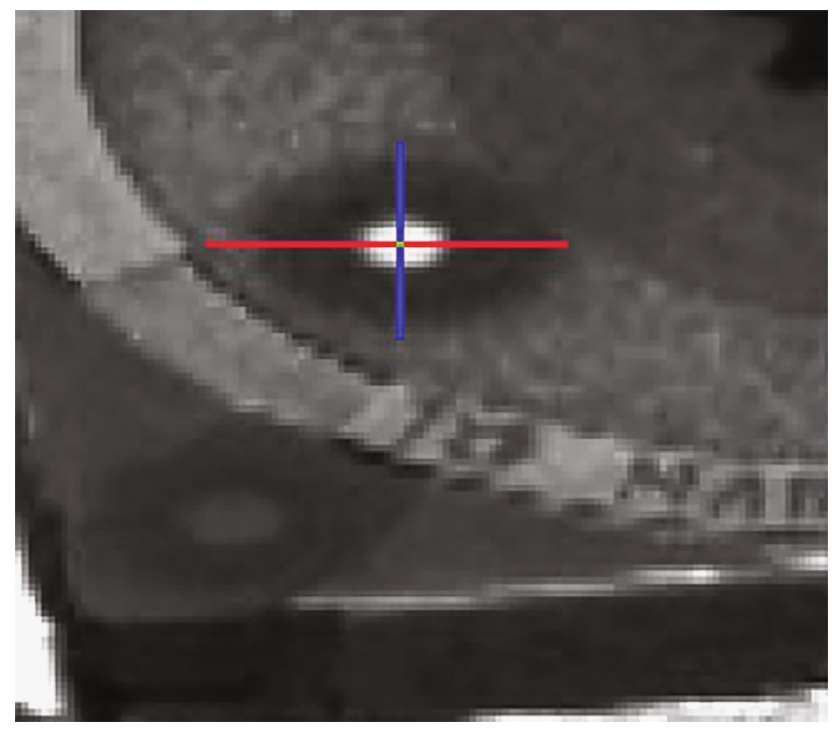

Fig. 18 Subframe of MSL Mastcam calibration target image acquired on sol 807 through the L2 (440 nm) filter. The subframe shows the clean spot produced by a sweep magnet under the white ring on the calibration target. An azimuthal (red) and an elevational (blue) profile are marked. Figure 19 shows data extracted from this image. Image ID: 0807ML0035390040400952D01_A2R1.IMG

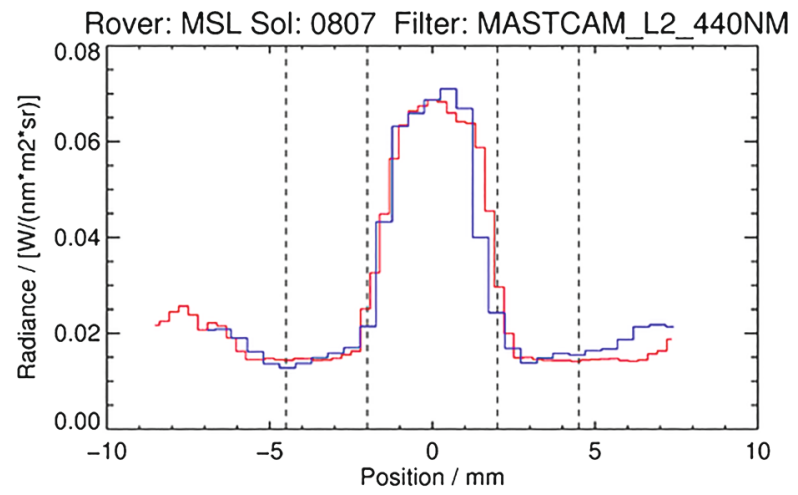

Fig. 19 Radiance values extracted along the horizontal and vertical transects of the sweep magnet marked on the image in Fig. 18. The transects are put on a common $\mathrm{x}$-axis of physical length (mm) which because of the perspective translates differently to number of pixels in the azimuthal versus the elevation direction. The dotted lines mark the position of the magnet. The plot demonstrates that the clean spot has a diameter of slightly more than $2 \mathrm{~mm}$

radiometric calibration coefficients used to convert from Data Numbers (DN) to radiance are a function of detector temperature and also have a straightforward dependence on focal length. The coefficients are summarized for a single temperature and two focal lengths in Table 7 of Hayes et al. (2021). These radiance-calibrated images are the starting point of the reflectance $(\mathrm{I} / \mathrm{F})$ calibration.

In the absence of the martian atmosphere converting from radiance to reflectance is a simple matter of dividing by the known solar irradiance as a function of wavelength and 


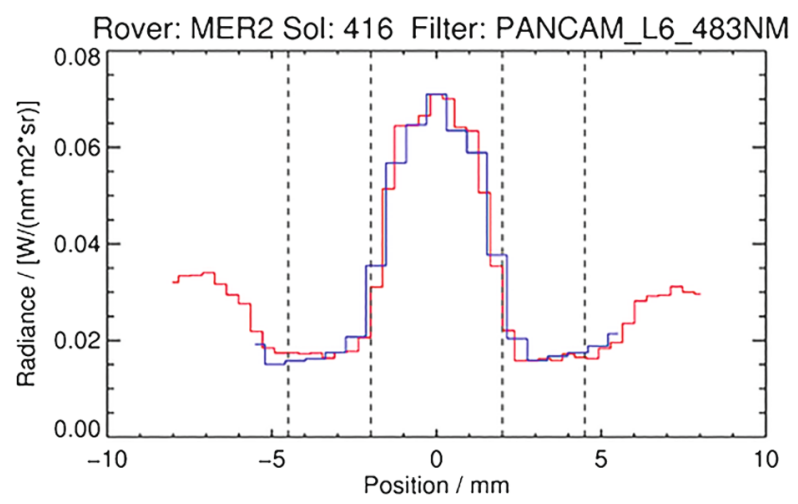

Fig. 20 Radiance values extracted along horizontal and vertical transects of the sweep magnet on the MER-A rover (Spirit) on sol 416 (image not shown). The transects are put on a common x-axis of physical length $(\mathrm{mm})$ which because of the perspective translates differently to number of pixels in the azimuthal versus the elevation direction. The dotted lines mark the position of the magnet. The plot demonstrates that the clean spot has a diameter of slightly more than $2 \mathrm{~mm}$

Mars-Sun distance. Figure 2 of Hayes et al. (2021) lists a reference DN level for each filter, defined as the DN level (after bias and dark current subtraction) expected from observing in a $10 \mathrm{~ms}$ exposure a perfectly diffuse and white sunlit surface at zero-incidence when Mars is at perihelion (1.38 AU) ignoring atmospheric attenuation. From these reference DN levels a rough reflectance estimate can be derived knowing only the exposure time and Mars-Sun distance.

\subsubsection{Principle of Reflectance (I/F) Calibration}

As pointed out in Sect. 2.2, the irradiance on the Martian surface is significantly affected by the dusty atmosphere, may change on short timescales, and is hard to model accurately. Thus, in order to produce "tactical", reflectance calibrated data products fast enough to inform daily commanding of the rover, we rely on images of the calibration targets.

A radiance-calibrated image of a martian scene is produced by a combination of the illumination, the reflectance of the scene, and the system spectral throughput. We can write this as:

$$
\text { Image }_{R A D}=\frac{1}{\pi} \int F(\lambda) \cdot r(\lambda) \cdot \rho(\lambda) \cdot d \lambda \sim \frac{F}{\pi} \int r(\lambda) \cdot \rho(\lambda) \cdot d \lambda=\frac{F}{\pi} \text { Image }_{\text {IOF }}
$$

Where $F(\lambda)$ is the spectral flux (energy per time per area perpendicular to the direction of light propagation), $\mathrm{F}$ is the Flux averaged over the band, $\mathrm{r}(\lambda)$ is the normalized system spectral response, and $\rho(\lambda)$ is the spectral reflectance, here given as $\mathrm{I} / \mathrm{F}$. The $\pi$ is just the conversion factor from bidirectional reflectance to $\mathrm{I} / \mathrm{F}$. The approximation of replacing the spectral flux with the average value is fundamental to our approach. For the narrow-band filters with bandwidths of a few tens of nanometers this not a large source of uncertainty. For the broad Bayer RGB filters it is more significant. Fundamentally, the IOF image is the scene reflectance folded with the camera system spectral throughput curve. Ideally one should fold also with the illumination spectrum, but since this is the unknown quantity, that is not possible - hence we approximate the illumination spectrum with an average over the band. 
When imaging the calibration target, the radiance from any given color and grayscale can be written in the same form as Eq. (2) above:

$$
C T_{R A D}=\frac{1}{\pi} \int F(\lambda) \cdot r(\lambda) \cdot \rho(\lambda) \cdot d \lambda \sim \frac{F}{\pi} \int r(\lambda) \cdot \rho(\lambda) \cdot d \lambda=\frac{F}{\pi} C T_{I O F}
$$

In this case, the I/F value of each calibration target material (material reflectance folded with the camera system spectral throughput curve) is known from pre-flight characterization (see Sect. 4). In Eq. (3), therefore, the flux F is the only unknown. This quantity can therefore be isolated and used in Eq. (2):

$$
\frac{\text { Image }_{R A D}}{C T_{R A D}} \sim \frac{\text { Image }_{I O F}}{C T_{I O F}}<=>\text { Image }_{I O F} \sim C T_{I O F} \cdot \frac{\text { Image }_{R A D}}{C T_{R A D}}
$$

Equation (4) is the basic equation behind the tactical I/F calibration procedure using the calibration target. In practice, as described in Sect. 5.4.3, there are several calibration target materials, each with their own pre-flight-characterized reflectance value and so we perform a linear fit, effectively averaging over several instances of Eq. (4), one for each reference material.

Note that multiplicative terms in the radiance calibration (DN-to-radiance coefficients) divide out of Eq. (4) to the extent that they are identical between the calibration target image and the image of a martian scene. Thus, potential radiance calibration problems such as drift in detector sensitivity etc. will not, to first order, affect the I/F calibration. Note also, that the method does not rely on similarity in illumination geometry between calibration target image and the image being calibrated. The calibration target reflectances are known in the specific illumination and observation geometry of the calibration target image (from the pre-flight reflectance model as described in Sect. 4). From this, the solar flux is known. The I/F calibrated image is then an expression of the I/F of the imaged scene in the specific illumination and observation geometry of that image.

Note also, since I/F (unlike $\mathrm{R}^{*}$ ) is fundamentally defined relative to flux (energy per area normal to direction of propagation), rather than irradiance (energy per area impinging on the surface), the derived $\mathrm{I} / \mathrm{F}$ is strictly correct, no matter the specific solar incidence of the observed surface. Of course, in order to convert to $\mathrm{R}^{*}$ and know the albedo of the surface, one needs to know the angle of incidence (see Eq. (1) for relation between $\mathrm{R}^{*}$ and $\mathrm{I} / \mathrm{F}$ ).

\subsubsection{Practical Implementation of the I/F Calibration}

For each calibration target image, Regions of Interest (ROI's) will be selected for analysis. The selection will happen by a semi-automated process. First, a predefined pattern is automatically overlain on the image using known pointings and zoom settings combined with minor adjustment by an image-alignment algorithm. Second, these patterns will be manually checked by a calibration pipeline operator and adjusted if necessary. The relatively dust-free central spots of the eight color and grayscale patches will be selected as will illuminated regions of the four grayscale rings. In addition, shadowed regions of the grayscale rings will be selected.

The observed mean radiances from selected ROI's in direct sunlight are then plotted as a function of known substrate reflectance values in the same illumination and viewing geometry. Figure $21 \& 22$ show examples of such radiance-reflectance plots based on calibration target images acquired during ATLO. For more examples see e.g. Bell et al. (2006).

With a clean calibration target the observed ROI mean radiance values fall on a straight line through the origin in such a radiance-reflectance plot and the slope of this line is a 

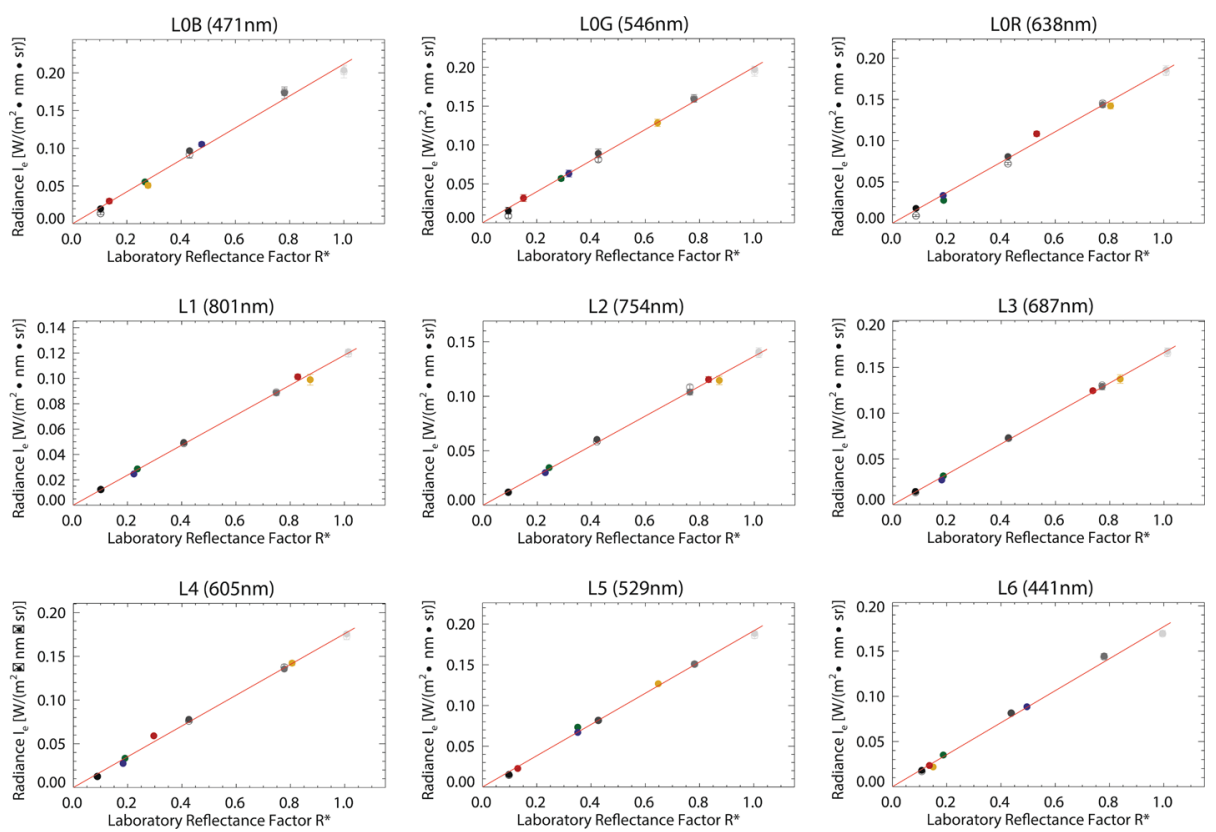

Fig. 21 Radiance-reflectance plots for all left-eye Mastcam-Z filters. Based on calibration target images acquired at $27 \mathrm{~mm}$ focal length during ATLO on October 26th. This is the same dataset as depicted in Fig. 16. As described in Sect. 4.4, we included an offset term in the linear fit, to effectively perform bias subtraction by comparison to the calibration target. In these figures, the offset term has been subtracted before plotting. Red lines are best-fit lines through the origin. The slope of this line is a measure of the irradiance

measure of the irradiance at this wavelength (determined by the filter used for acquisition of this particular image).

Each of the 8 color and grayscale patches and each of the 4 grayscale rings generate a data point on the plot. A least-squares fit is performed to determine the slope of the best-fit line and derive a value for the irradiance, which is effectively a translation from units of radiance to units of reflectance. This procedure is similar to the procedure employed for MER Pancam (Bell et al. 2003) and MSL Mastcam (Bell et al. 2017).

\subsubsection{Diffuse Sky Light}

The procedure described in Sects. 5.4.3 and 5.4.4 assumes the illumination to be fully directional, i.e. all light arriving from the direction of the sun. In reality, on Mars, a significant fraction of the light is scattered light from the atmosphere. The fraction of the total irradiance that is diffuse skylight varies from $\sim 20 \%-30 \%$ around noon under relatively low atmospheric dust loading to essentially $100 \%$ under dust storm conditions (Kinch et al. 2015). This complicates derivation of I/F in two ways: First, Since the reflectance of the calibration target materials depends on illumination geometry, it may be slightly different for diffuse sky illumination than for direct solar illumination. Second, since any measure of reflectance must be defined relative to an illumination and observation geometry, strictly the derived I/F of the martian scene will be a reflectance under the specific combination of direct sunlight and diffuse sky illumination that was present when the image was acquired. 

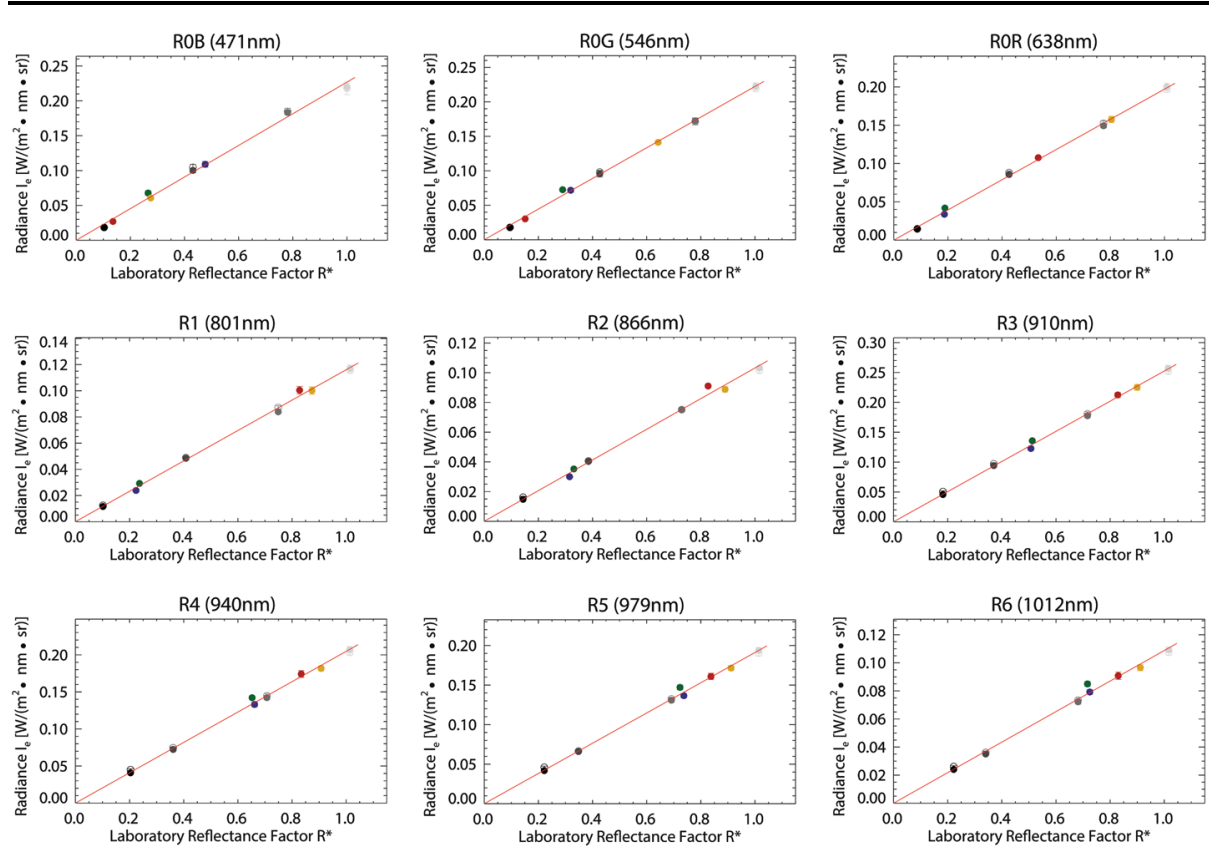

Fig. 22 Radiance-reflectance plots for all right-eye Mastcam-Z filters. Based on calibration target images acquired at $27 \mathrm{~mm}$ focal length during ATLO on October 26th. This is the same dataset as depicted in Fig. 16. As described in Sect. 4.4, we included an offset term in the linear fit, to effectively perform bias subtraction by comparison to the calibration target. In these figures, the offset term has been subtracted before plotting. Red lines are best-fit lines through the origin. The slope of this line is a measure of the irradiance. The lamp was moved before acquiring the longest-wavelength filters, hence the jump in slopes between R2 and R3

The way we handle this problem in the calibration pipeline is by approximating the illumination as consisting of two components: One directional, from the Sun, one diffuse, arriving equally from the entire sky hemisphere. For each calibration target material, the model returns two values of $\mathrm{R}^{*}$ : One directional for the relevant geometry, and one hemispherical, found from an average over all angles of incidence and azimuth. The shadowed regions of the grayscale rings are then modeled as only illuminated by the diffuse component, while all other surfaces are illuminated by both the diffuse and the directional component. In this way the model will derive two free parameters describing the total irradiance: $\mathrm{J}=\mathrm{J}_{\text {Directional }}+$

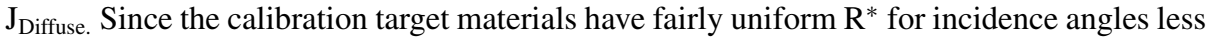
than $\sim 45^{\circ}$ (see Sect. 4.2), and since most imaging will happen with the sun relatively high in the sky, in most cases the calibration target reflectance estimate will not be radically different between the directional and the diffuse component. Problematic situations may occur at low sun, when the diffuse skylight is very far from uniformly distributed across the sky, especially in a forward-scattering geometry, when many of the calibration target materials have strong reflectance peaks (see Sect. 4.2).

Conceptually, the presence of the diffuse component of illumination presents another problem in interpreting the $\mathrm{I} / \mathrm{F}$ calibrated images. The solar flux $\mathrm{F}$ is an inherently directional quantity. With a diffuse component present, the illumination must instead be expressed in terms of irradiance $\mathrm{J}=\mathrm{F} \cdot \cos (\mathrm{i})$. In this formulation, the angle of incidence of the sunlight on the surfaces in the image matter for the interpreted $\mathrm{I} / \mathrm{F}$ value and so this value is now only strictly valid for surfaces with the same angle of incidence as the calibration target 
(essentially, horizontal surfaces). Another way of expressing this conceptual problem: With purely directional illumination the relation between irradiance and angle of incidence is well understood. With a diffuse component present that in reality is not evenly distributed across the sky, the irradiance on any surface that is not level with the calibration target is not that well-known, which will introduce extra uncertainty in interpreting the derived I/F values when applied to non-horizontal surfaces.

\subsection{Dust Correction}

Dust is always present in the martian atmosphere. As soon as the Perseverance rover has landed the rover deck, including Mastcam-Z calibration targets, will begin accumulating a layer of aeolian dust. In addition, as discussed in Sect. 2.2, an appreciable amount of material is likely to be deposited during the landing process as the skycrane retrorockets mobilize surface materials. Notably, this component may have different color properties from the aeolian dust. Because of the overlying dust, the reflectance properties of the calibration target materials will begin changing immediately after landing and will no longer be fully described by the pre-flight characterization (Sect. 4). This represents a significant problem for reflectance calibration. We plan to handle the problem of dust deposition by two separate methods. First, through the built-in sweep magnets, that will significantly reduce dust deposition on sections of the primary calibration target. Second, by a dust-correction algorithm that has been used successfully on MER (Kinch et al. 2015) and MSL (Bell et al. 2017).

As was described in Sect. 5.2, because of the magnetic properties of martian aeolian dust, the strong magnets built in to the primary calibration target are expected to very significantly reduce dust deposition in the central spots of the eight circular color and grayscale patches. Extensive experience from MER, Phoenix and MSL indicates that these spots will remain relatively dust-free. They will not be entirely clean, as some non-magnetic dust will accumulate both during landing, as the magnetic properties of ground-derived materials deposited during landing are not known, and later, since there is a small non-magnetic component of the aeolian dust. The four central grayscale rings are expected to accumulate significantly more dust as the mission progresses.

In order to correct for the presence of dust we will employ a method described in full detail by Kinch et al. (2015), validated in laboratory studies by Johnson et al. (2006) and inflight on MER (Kinch et al. 2015) and MSL (Bell et al. 2017). The dust correction procedure fits the observed radiance values to an analytical two-layer scattering model based on the work of Hapke (1993, Sect. 9.D.2). The model treats single-scattering events in full detail and uses a two-stream formalism (e.g., Zdunkowski et al. 2007) to treat multiple-scattering events. Below we will go into some detail about the dust correction procedure, to some extent repeating and paraphrasing material found in Kinch et al. (2015). For full detail that work should be consulted.

\subsubsection{The Problem}

The presence of dust on the calibration target is easily observed in calibration target images as a reduction in contrast between the bright and the dark surfaces. The dustier the target gets, the more the observed radiance from all materials will approach the same value. Thus, by employing a suitable mathematical model, an estimate of dust thickness can be derived from each single calibration target image. Using even very simple models a rough but reasonable estimate can be derived frame-by-frame and in the absence of any information about dust color properties. 
The harder problem is to understand what effect the dust has on the reflectance value, and thus on the derived irradiance - the quantity needed to perform the reflectance calibration. The presence of dust can be detected and the amount estimated from the reduction in contrast, but from single frames it is very hard to tell the difference between bright dust under relative low irradiance and darker dust under brighter irradiance. In Sect. 5.5.2, we describe the analytical two-layer reflectance model used in our dust correction algorithm, while Sect. 5.5.3 describes the method used to derive a reflectance dust spectrum.

\subsubsection{Dust Model}

The choice of model description is a balance between having a model complex enough to adequately reproduce the reflectance behavior of the dusty calibration target while simple enough and relying on a small enough number of parameters that it lends itself to routine application of a best-fit procedure for parameter determination. The model we use to describe directional scattering from a dust-covered calibration target surface is essentially the same as that given by Hapke (1993, Sect. 9.D.2) for reflectance of a double-layer of particulate material, except we have rewritten the equations so that the lower layer is not described as a layer of particulates but rather described as a single entity with reflectance properties given by our calibration target reflectance model (see Sect. 4). For the diffuse illumination component (see Sect. 5.4.4) we use the diffusive-reflectance model also given by Hapke (1993) Eq. 9.14 based on the two-stream formalism (e.g., Zdunkowski et al. 2007). At the highest level, the model can be written as:

$$
\begin{aligned}
C T_{R A D}= & \frac{J_{\text {Direct }}}{\pi} R_{\text {Direct }}^{*}\left(\boldsymbol{\tau}_{M}, \boldsymbol{w}_{M}, \text { Material }, \text { Filter }, i, e, g\right) \\
& +\frac{J_{\text {Diffuse }}}{\pi} R_{\text {Diffuse }}^{*}\left(\boldsymbol{\tau}_{M}, \boldsymbol{w}_{M}, \text { Material }, \text { Filter }\right)
\end{aligned}
$$

The full equations are given in Kinch et al. (2015) and will not be repeated here.

Of the quantities listed in the above equation, material (i.e. calibration target substrate), filter, and the angles i, e, g, are all specified by the conditions of the measurement. The four remaining quantities, listed in bold above, are the diffuse and directional components of the irradiance, J, the optical depth of dust deposited on the calibration target, $\tau_{M}$, and the singlescattering albedo of the dust, $\mathrm{w}_{\mathrm{M}}$. Both $\tau_{M}$ and $\mathrm{w}_{\mathrm{M}}$ should be thought of as proxies within the limited framework of the scattering model for the true microphysical optical depth and single-scattering albedo. Both parameters are sensitive e.g. to assumptions about the phase function of single-scattering events and more generally the mathematical radiative-transfer formalism is based on assumptions about widely separated dust grains that are manifestly not true in this application. The subscript $\mathrm{M}$ is intended as a reminder that the relation between this model parameter and the true microphysical quantity is far from straightforward (see e.g. Shepard and Helfenstein (2007)). Our goal here is not to derive microphysical quantities of deposited dust, but rather to accurately reproduce the reflectance of a dusty calibration target surface.

In the implementation (see Sect. 5.5.4.) three quantities are derived for each image, these are the irradiance, split into a direct component from the Sun $\left(\mathrm{J}_{\text {Direct }}\right)$ and a diffuse sky component $\left(\mathrm{J}_{\text {Diffuse }}\right)$, and the model dust optical depth $\left(\tau_{\mathrm{M}}\right)$. The last quantity, the model dust single-scattering albedo $\left(\mathrm{w}_{\mathrm{M}}\right)$, must be specified. This quantity is discussed in Sect. 5.5.3 below. 
Fig. 23 Spectra of model dust single-scattering albedo values derived by the procedure of Kinch et al. (2015) and outlined in Sect. 5.5.3. The MER curves are reproduced from Kinch et al. (2015) while the Curiosity curve is derived by the same procedure applied to data from the Curiosity Mastcam (Bell et al. 2017)

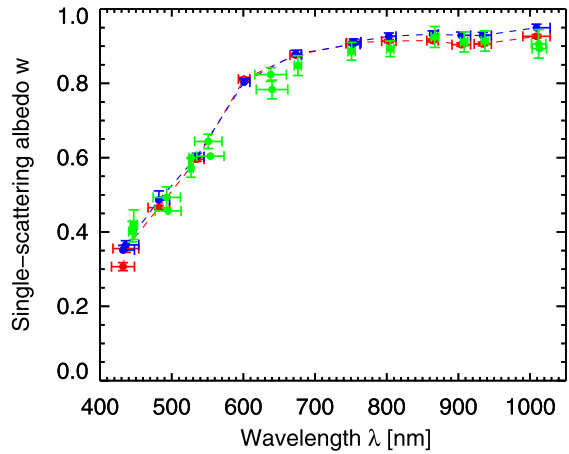

\subsubsection{Dust Spectrum}

As described in Sects. 5.5.1 and 5.5.2, the model dust single-scattering albedo cannot be disentangled from the irradiance based on single image frames and so must be specified in order to derive irradiance. In Kinch et al. (2015) a procedure is outlined for deriving this dust single-scattering albedo from a simultaneous analysis of a large number of calibration target images acquired over many sols with varying thicknesses of deposited dust. The idea is to look for correlation between model optical thickness of deposited dust and derived irradiance. If the model dust single-scattering albedo is too low, the derived irradiances will be observed to (artificially) increase as the calibration target gets dustier. Conversely, if the model dust single-scattering albedo is too high, the derived irradiances will be observed to (artificially) decrease as the calibration target gets dustier. The correct model dust singlescattering albedo is the value that causes no correlation between deposited dust thickness and derived irradiance.

This procedure was successfully employed for data from the MER Spirit and Opportunity rovers (Kinch et al. 2015) as well as for the Curiosity rover (Bell et al. 2017). The derived dust spectra for all three missions are shown in Fig. 23. Note that these values are derived independently for each filter on each mission. The results are very consistent in terms of derived dust spectra. For the early mission, we will employ a dust reflectance spectrum based on a smoothed average of the values derived in these earlier missions. Later on, as data accumulates, we will be able to derive a dust spectrum directly from the Mastcam- $Z$ dataset (in fact, two dust spectra, since it is not obvious that non-magnetic dust in the magneticallyprotected patch centers will have the same color properties as dust that accumulates on the grayscale rings).

\subsubsection{Dust Correction Implementation}

Figure 24, reproduced from Kinch et al. (2015), shows an example of the implementation of this model to an L4(601 nm) Pancam calibration target image from sol 180 of MER Spirit. The left frame shows radiances observed from the dusty target as a function of model clean substrate reflectances. The reduced contrast can be seen as the relative difference between bright and dark substrates is reduced in the observation (y-axis) relative to the model ( $\mathrm{x}-$ axis) and the data points do not fall on the predicted straight line through the origin. After fitting to the dust model, the right frame shows the same observed data (y-axis) now plotted relative to the dust model prediction for the reflectances. The linear relation is recovered. Note the presence of the shadowed data points (open circles) in both figures, falling on a separate straight line in the right frame, indicative of the level of diffuse sky illumination. 

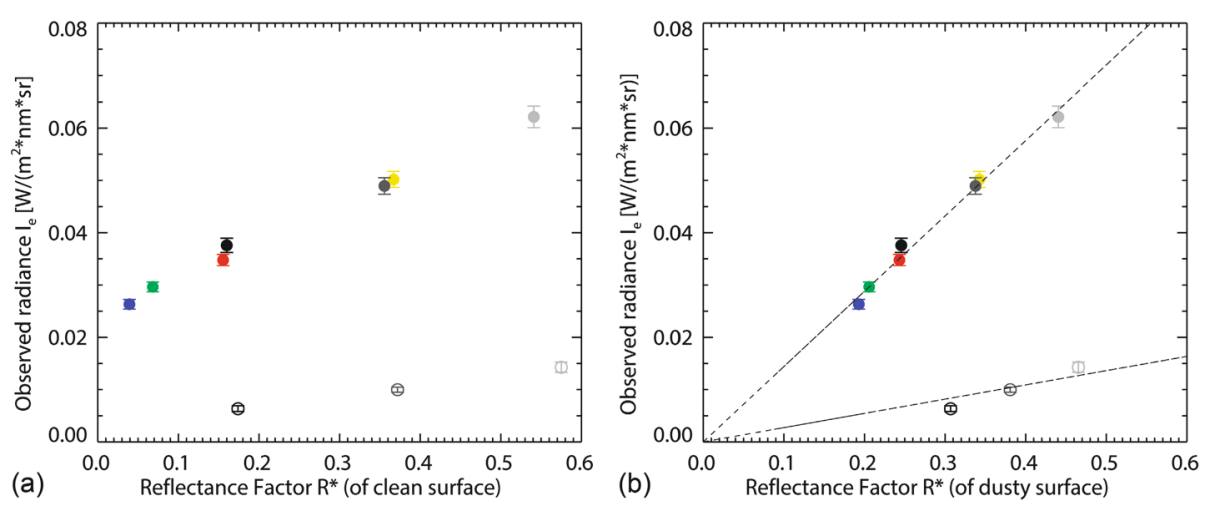

Fig. 24 Reproduced from Figs. 2, 3 of Kinch et al. (2015). Illustration of implementation of the dust-correction algorithm here on data from a calibration target image acquired on sol 180 of the Spirit rover mission. a) Mean radiances derived from 7 sunlit ROI's (filled circles) and 3 shadowed ROI's (open circles) as a function of pre-flight derived reflectance estimates. b) The same mean radiance values, now as a function of dust-model derived reflectance values

The model derives for each image independently values for the directional and diffuse irradiance together with a value for the optical thickness of deposited dust. Since we may expect the dust thickness to be different in the magnetically-protected color and grayscale patches from the value on the grayscale rings we may perform the fit independently for patches and rings and compare derived irradiance estimates. The irradiance would be expected to be identical for patches and rings, so the comparison can be a check of the quality of the data. Alternatively, we may construct a fit to all extracted ROI's with two free parameters for two different dust thickness values but only one free parameter for the derived irradiance, and thus force the irradiance estimate to be consistent between patches and rings.

\subsection{Error Estimates}

The pre-flight calibration of the Mastcam- $Z$ flight instrument and resulting estimates of the uncertainty on calibrated data products is described in detail in Hayes et al. (2021). In Sects. 3.1.1 and 4.1.7 of that work, it is demonstrated that reflectance-calibrated Mastcam-Z images agree with spectra of calibration target materials as well as a number of laboratorycharacterized rock samples to within 5\% RMS in all cases - in many cases significantly better. This is under controlled laboratory conditions, though. The martian environment will introduce further sources of uncertainty such as

- Changes to irradiance in the time between acquisition of the image and acquisition of the associated calibration target image

- Uncertainties introduced by the presence of dust on the target (see Sect. 5.5)

- Uncertainties associated with the angular distribution of diffuse illumination from the sky (see Sect. 5.4)

Other contributions to the uncertainty are associated with additive terms in the radiance calibration (bias subtraction) and with uncertainty of the calibration target reflectance model (Sect. 4). On the other hand, I/F products will to first order be unaffected by uncertainties or drift in the multiplicative terms of the radiance-calibration pipeline (see Sect. 5.4.2)

Especially the uncertainties associated with the martian environment are hard to quantify and work will be ongoing in-flight to assess these uncertainties by comparison of in-flight 
calibration target images to pre-flight spectra as well as by observation of other targets of relatively well-known reflectance (SuperCam calibration target, rover deck, thick surface dust deposits, targets also observed with e.g. the SuperCam passive spectrometer).

The main goal of the calibration target is for rapid tactical calibration to influence the next sols activities. Our expectation is that later, after more detailed in-flight verification, more detailed model development, and more detailed image processing, higher-fidelity I/Fcalibrated images can be produced enabling longer-term science output from the investigation (e.g. Wellington et al. 2017).

\subsection{Cross-Comparison Between Primary and Secondary Targets}

The analysis described in Sect. 5.4 and 5.5 may be employed also for the secondary calibration target as an extra independent verification. At times the secondary target will be in shadow, and irradiance on the secondary target will be different from that experienced by the primary target. When the secondary target is in direct sunlight, primary target patches and rings, and horizontal secondary target surfaces should all result in different derived dust thicknesses but the same irradiance value.

In addition to the use for calibration the dust-correction procedure will derive a history of dust thickness on the calibration targets in which seasonal variation in dust lifting and deposition can be tracked. Also, comparison between the magnetically-protected regions on the patches and the grayscale rings and secondary target surfaces will yield an estimate of the fraction of the aeolian dust grain population that is non-magnetizable. A separate derived estimate of dust thickness on the vertical surfaces of the secondary target can be used to analyze the relative importance of different dust deposition processes (gravitational settling versus Brownian motion random walk or electrostatic attraction).

\subsection{Cross-Comparison with SuperCam}

The SuperCam instrument light gathering telescope (Wiens et al. 2020, this issue, Maurice et al. 2020, this issue) is mounted on the rover mast above the Mastcam-Z. The SuperCam calibration target (Manrique et al. 2020, this issue) is on the rover deck above and behind the RPFA-box and the Mastcam-Z calibration targets (see Fig. 3). The SuperCam calibration target contains a set of five magnetically-protected optical patches analogous to the patches on the Mastcam-Z primary target intended for calibration of the SuperCam passive visiblenear-infrared spectroscopy mode and the SuperCam remote micro-imager (RMI). Four of these patches reproduce materials present in the Mastcam-Z target (AluWhite98 and Lucideon Cyan, Green and Red) while the fifth is painted with an IR-black paint (Aeroglaze Z307).

The SuperCam calibration target is visible to the Mastcam- $Z$ and the patches can be imaged using Mastcam-Z. Likewise, the Mastcam- $Z$ calibration targets may be observed with the SuperCam passive and RMI modes, although with some focus limitations. These targets allow a dedicated cross-calibration program between Mastcam- $Z$ and SuperCam passive and RMI observations to assure internal consistency, as has been done between the analogous Mastcam and ChemCam instruments on the MSL rover Curiosity (Johnson et al. 2015).

\section{Summary}

The Mastcam-Z instrument (Bell et al. 2021) is a Mast-mounted, stereoscopic, multispectral camera with zoom capability. Mastcam- $Z$ raw images are converted to physical units of 
radiance based on pre-flight-acquired calibration data (Hayes et al. 2021). Mastcam-Z relies on a set of radiometric calibration targets for tactical in-flight conversion of radiance data to units of reflectance $\left(\mathrm{R}^{*}\right.$ or $\left.\mathrm{I} / \mathrm{F}\right)$. The Mastcam-Z primary radiometric calibration target is a high-heritage evolutionary development drawing on design elements from calibration targets on the MER, Phoenix and MSL missions. The Mastcam-Z secondary radiometric calibration target is a simple design intended to augment and validate the calibration-related information derived from the primary target. We have described the design considerations that led to the final designs for these two targets. Finally, we have described the pre-flight optical characterization of the targets and discussed their use during flight for tactical-timeline conversion of Mastcam-Z images of Mars from units of radiance to units of reflectance. Appendices A \& B describe in detail the manufacture of the targets and provide information about the test program of dynamical and thermal tests.

Acknowledgements This project was supported by the Carlsberg Foundation grants CF16-0981, CF170979, and CF19-0023. The project has also benefitted enormously from the technical and financial support of the Mars 2020 Project, Payload, and Science Offices at JPL. M. Merusi is supported by the European Union's Horizon 2020 research and innovation programme under the Marie Skłodowska-Curie grant agreement No 801199. Thanks to Jesse Kuik, Alexis Parkinson, Nathalie Turenne, and Evan Stanish for their assistance with calibration target and Mastcam-Z testing at the University of Winnipeg. Calibration target testing at the University of Winnipeg was supported by the Canadian Space Agency (CSA), the Natural Sciences and Engineering Research Council of Canada (NSERC), the Canada Foundation for Innovation (CFI), the Manitoba Research Innovations Fund (MRIF), and the University of Winnipeg. Acknowledgements to FORCE Technology for providing flight acceptance vibration test for the 2nd set of flight spare target free of charge, and to DuPont Electronic Materials for providing the Kapton ${ }^{\circledR}$ foils used as spacers during assembly of Primary Calibration Targets.

Publisher's Note Springer Nature remains neutral with regard to jurisdictional claims in published maps and institutional affiliations.

Open Access This article is licensed under a Creative Commons Attribution 4.0 International License, which permits use, sharing, adaptation, distribution and reproduction in any medium or format, as long as you give appropriate credit to the original author(s) and the source, provide a link to the Creative Commons licence, and indicate if changes were made. The images or other third party material in this article are included in the article's Creative Commons licence, unless indicated otherwise in a credit line to the material. If material is not included in the article's Creative Commons licence and your intended use is not permitted by statutory regulation or exceeds the permitted use, you will need to obtain permission directly from the copyright holder. To view a copy of this licence, visit http://creativecommons.org/licenses/by/4.0/.

\section{Appendix A: Manufacture}

\section{A.1 Primary Target}

\section{A.1.1 Bases, Plating and Engraving}

The base structures of the Mastcam-Z primary calibration targets were machined from a 100 mm diameter rod of Al-6082-T6 using a CNC cutter at the Niels Bohr Institute mechanical workshop. Figure 4 shows a drawing of the Mastcam-Z primary calibration target parts and how these were assembled into one single unit using different types of epoxy adhesives depending upon which combination of materials had to be bonded.

The base structure has essentially an octagonal symmetry, but the four mounting ears and weight reducing cutouts of material from the bottom reduces the symmetry slightly. From the top, 8 symmetrically placed cutouts were made to fit ring magnets of dimensions 
(nominally) $11.0 \mathrm{~mm}$ outer diameter, $5.0 \mathrm{~mm}$ inner diameter, and $7.0 \mathrm{~mm}$ height. A small stub in the center of the cutout for magnets support the base of the reference color ceramic inserts

A central cylindrical cutout, $50.35 \mathrm{~mm}$ outer diameter, $9.85 \mathrm{~mm}$ inner diameter and 3.0 $\mathrm{mm}$ deep, accommodates the four concentric color reference ceramic rings, and the central remaining structure serves as a support of the central shadow post (gnomon). Figure 4 shows the geometry of the cutouts in the base structure to accommodate the ring magnets, the ceramic rings, and the gnomon.

We wished the completed calibration target assembly to have a matte finished golden surface with surface embellishments (engraved decorations) of a contrasting color. This was achieved by first matting the entire aluminum structure by glass bead blasting and ultrasonic cleaning before subsequently silver plating as a basis for a thin layer of gold plating. This two-step process was used to ensure good adhesion of the surface plating materials to the aluminum structure material. The black color of the engravings comes from oxidation of the silver to oxidize in air during the laser engraving process, and does not involve any coloring agents.

The plating of the base structures was done at SUR-TECH A/S, a surface technology company in Farum, Denmark. Laser engravings were done at Andersson \& Sørensen in Ishøj, Denmark using a rofin CL-Basic laser engraver. The first series of units were manufactured with (nominally) $10 \mu \mathrm{m}$ of silver below a plating of $3 \mu \mathrm{m}$ of gold. The laser engraving should burn away the thin layer of gold on top of the silver plating in the process oxidizing the silver to black silver oxide. However, after a breakdown of the laser engraver initially used for tests it turned out to be difficult to find the parameters of the setup that penetrated the gold, but did not burn away any of the silver plating. Therefore, a second batch of structures were manufactured with (nominally) $40 \mu \mathrm{m}$ of silver below a plating of $3 \mu \mathrm{m}$ of gold.

\section{A.1.2 Magnets}

Eight ring-shaped $\mathrm{Sm}_{2} \mathrm{Co}_{17}$ alloy permanent magnets, magnetized along the axis of symmetry, were built into the primary calibration target. The magnets are hollow cylinders with outer diameter $11 \mathrm{~mm}$, inner diameter $5 \mathrm{~mm}$, and height $7 \mathrm{~mm}$ and were produced by the company Vacuumschmeltze in Germany (www.vacuumschmeltze.com). All magnets have been pre-aged at $150{ }^{\circ} \mathrm{C}$ for thermal stabilization as part of their production.

\section{A.1.3 Color Patches and Rings}

Selection of color and grayscale materials initially involved examining a range of possible materials for use as white, gray, black, and color standards. During these tests possible target materials were exposed to a combination of vacuum, a Mars-like $\mathrm{CO}_{2}$-rich atmosphere at average Mars surface pressures, with and without broadband UV illumination using a deuterium light source at the University of Winnipeg's Center for Terrestrial and Planetary Exploration (C-TAPE). For the eventual target assembly, we chose materials that showed no or the least change in reflectance after these tests.

The white ring and white patch are made from AluWhite98 sintered alumina provided by Avian technologies in the US (www.aviantechnologies.com). The rest (all colors, blacks, and greys) are made from glazed and matted aluminum-silicates provided by Lucideon in the UK (www.lucideon.com). The names of the 7 materials provided by Lucideon are Black, 
Grey33, Grey70, Cyan, Green, Bright Yellow and Red. Here, we refer to them as Black, Dark Grey, Light Grey, Blue, Green, Yellow and Red.

All rings and patches were subjected to $>48$ hours bakeout at $110^{\circ} \mathrm{C}$ in a vacuum $(\mathrm{p}<$ $10^{-2} \mathrm{~Pa}$ ) prior to being mounted in the calibration target structure, in order to remove any trace of cutting fluids.

\section{A.1.4 Shadow Post}

Like the base, the central shadow post, the gnomon, was machined at the Niels Bohr Institute mechanical workshop from Al 6082-T6 using a CNC cutter. It was then matted by glass bead blasting and painted with Aeroglaze Z307, a conductive black paint of ultralow reflectivity throughout the visible and infrared range of wavelengths.

\section{A.1.5 Assembly Process}

All assembly was done in a flow bench in a class 10000 clean room at the Niels Bohr Institute, University of Copenhagen.

A batch of adhesive (Henkel/Hysol EA956) was thoroughly mixed inside a sealed bag. After mixing the adhesive, it was moved to a plastic container which in turn was placed in a thermal bath to dissipate heat from the mixing to maintain a comfortably long pot life of the adhesive. A thin layer of Henkel/Hysol EA956 adhesive was applied to the bottom and outer rim of magnets and these were mounted into the structure and rotated when inserted to evenly distribute the adhesive. The magnets were mounted in a pattern with alternating polarity (i.e., south up, north up, south up, etc.) in order to minimize stray magnetic fields.

Insertion of magnets into the structure was done with the aluminum structure lying on a sheet of Teflon on a magnetizable steel plate in order to stabilize the magnets in the bottom of their individual cavities. This held the magnets gently in place and prevented them from moving out of the cavities under their mutual magnetic force. The magnetic force from the steel plate was gentle enough to still allow the magnets to be rotated carefully when mounted as described above. There are small vent holes in the bottom of the aluminum structure to allow any excess adhesive to escape through the bottom, and to allow outgassing from the bond line during final bakeout $\left(>110^{\circ} \mathrm{C}\right.$, at least 48 hours in a vacuum, see Appendix B, Sect. B.2.2).

The structure with magnets was allowed to cure for at least 24 hours at room temperature before mounting of the ceramic color tiles.

The adhesive used for bonding the color and grayscale patches into the cylindrical magnets was Henkel/Loctite EA9309NA. Following the same procedure as when mounting magnets, the two-component adhesive was thoroughly mixed inside a sealed bag. After mixing the adhesive, it was moved to a plastic container which in turn was placed in a thermal bath to dissipate heat from the mixing to maintain a comfortably long pot life of the adhesive.

A thin layer of adhesive was applied first to the bottom of the head (lip) of the patches and then to the cylindrical part of the stub. Also, a thin layer of adhesive was applied to the inner surface (cylindrical hole) of the magnets. The patches were then carefully positioned into the magnets, rotated a bit around the axis of symmetry, and pushed gently into their final position.

The concentric ceramic grayscale rings were mounted directly on the basic structure of the Mastcam-Z primary calibration target using a continuous band of 3M2216B/A Gray, an epoxy adhesive suitable for bonding ceramics to metals. The $3 M 2216 B / A$ adhesive was mixed and poured out into a small plastic beaker while the amount of adhesive was measured 
on a scale. Immediately after, $1 \%$ by weight of $175 \mu \mathrm{m}$ spacer grade glass spheres were added to the $3 M 2216 B / A$ Gray adhesive used for the ceramic rings and thoroughly mixed into the adhesive. These glass spheres were added to help control bond line thickness. The plastic beaker was then placed in a cooler with water/ice-bath in order to dissipate heat from the mixing and thereby maintain a comfortably long pot life of the adhesive.

The four central rings of the Mastcam- $Z$ primary calibration target were mounted upside down on a centering tool and the adhesive was applied in an even layer to the bottom of the grayscale rings. Subsequently the set of rings were positioned in place in the aluminum structure and pressed to reach the correct bond line thickness (determined by the spacer grade glass spheres). The central rings were rotated slightly in place to ensure an even distribution of adhesive on both of the bonding surfaces.

Immediately after positioning of the rings in the structure, shims of Kapton ${ }^{\circledR}$ foil were inserted to ensure a co-axial centering of the rings in the structure. The outermost Kapton ${ }^{\circledR}$ shims, between the outer ring and the calibration target body, were $128 \mu \mathrm{m}$ thick, the three central shims, between the rings, were $27 \mu \mathrm{m}$ thick, and the innermost shims, between the inner ring and the central structure holding the gnomon were $53 \mu \mathrm{m}$ thick. A slight pressure was applied to the rings while they were curing by placing a set of dedicated thick Teflon rings on top of the grayscale rings and a beaker of water on top of this.

The adhesive was allowed to cure at room temperature. To follow the hardening of the adhesive, the small beaker with the unused adhesive was removed from the cooler and its curing was periodically tested with a plastic toothpick. When the adhesive had been observed to solidify in the beaker, the inserted Kapton ${ }^{\circledR}$ foils were gently removed while the surrounding rings were kept in place using pliers with Teflon ends.

Finally, after the $3 M 2216 B / A$ epoxy adhesive had cured for 24 hours, the shadow post was mounted using a small conical fastener from below and the thread was fixed with a drop of epoxy adhesive EA-956 mixed in the same way as described above for the application of this epoxy when mounting the magnets.

\section{A.2 Secondary Target}

\section{A.2.1 Base}

As was the case for the primary target, the base structures of the Mastcam-Z secondary calibration targets were machined from a plate of Al-6082-T6 using a CNC cutter at the Niels Bohr Institute mechanical workshop. After machining the base was matted by bead blasting

\section{A.2.2 Color Chips}

The seven different reference color chips used for the secondary Mastcam- $Z$ calibration target are all identical to materials used in the primary target. Every color in the primary target is reproduced in the secondary target, except yellow. The white chips are made from AluWhite98 sintered alumina provided by Avian technologies in the US (www.aviantechnologies.com). The rest (all colors, blacks, and greys) are made from glazed and matted aluminum-silicates provided by Lucideon in the UK (www.lucideon.com). The names of the 6 materials provided by Lucideon are Black, Grey33, Grey70, Cyan, Green and Red. Here, we refer to them as Black, Dark Grey, Light Grey, Blue, Green and Red. All chips were subjected to $>48$ hours bakeout at $110{ }^{\circ} \mathrm{C}$ in a vacuum $\left(\mathrm{p}<10^{-2} \mathrm{~Pa}\right.$ ) prior to being mounted in the base structure, in order to remove any trace of cutting fluids. 


\section{A.2.3 Assembly Process}

All assembly was done in a flow bench in a class 10000 clean room at the Niels Bohr Institute, University of Copenhagen. The adhesive used for bonding the square ceramic tiles into the aluminum metal is Henkel/Loctite EA9309NA. Following the same procedure as for the primary target, the two-component adhesive was thoroughly mixed inside a sealed bag. After mixing the adhesive, it was moved to a plastic container which in turn was placed in a thermal bath to dissipate heat from the mixing to maintain a comfortably long pot life of the adhesive.

For mounting of the square ceramic tiles for the Mastcam-Z secondary calibration target, a thin layer of adhesive was applied to the bottom of each tile and the tiles were positioned in place and pressed against the aluminum structure. No weight was applied during curing. During curing the target was resting horizontally on the surface that is vertical in the flight configuration. Thus, during curing, the horizontal and vertical orientation was switched.

There are small vent holes in the bottom of the cutouts in the aluminum structure to allow any excess adhesive to escape through the bottom - and to allow outgassing from the bond line during the final bakeout $\left(>110^{\circ} \mathrm{C}\right.$, at least 48 hours in a vacuum).

\section{A.3 Overview of Manufactured Calibration Target Units}

A total of 20 primary target bases were machined. During engraving each of these were engraved with a serial number. By error serial number 4 was skipped but otherwise the numbers run consecutively, so bases are numbered 1-21, skipping 4. As described in Sect. A.1.1 a first batch, serial numbers 1-11, were plated with a thinner $\sim 10 \mu \mathrm{m}$ layer of silver. Bases 12-21 were plated with the thicker $\sim 40 \mu \mathrm{m}$ layer of silver. Since the engraving process required some refinements, some bases had imperfect engravings, also challenges experienced during thermal testing required more targets to be assigned as Engineering Qualification Model (EQM) units than originally envisioned. Ultimately, targets were assembled by hand and the choice of which to designate as the flight unit was made after detailed inspection of assembled units. For this reason, the designation of targets as qualification models, flight spare, flight unit, etc does not follow a logical progression in serial numbers. Table 5 shows a list of all primary calibration target units assigned a serial number. "Dummy" magnets are nonmagnetic steel cylinders used for some units intended as optical references or exhibition units where strong magnetic fields were undesirable. Table 6 shows a similar list of secondary target units.

\section{Appendix B: Test Program}

The Mastcam-Z radiometric calibration targets were tested according to a Qualification Acceptance test program in which a set of Engineering Qualification Models (EQMs) were first subjected to qualification level vibration and shock tests followed by extensive thermal cycling in a qualification level "Packaging, Qualification and Verification" (PQV) test. The flight and flight spare units were subjected to a lower-level Flight Acceptance vibration test (no shock test) followed by three thermal cycles in vacuum (TVAC test). The order of testing follows the order the environments will be experienced by the flight targets: Vibration (launch) followed by shock (landing) followed by PQV/TVAC (surface operations).

The first set of EQMs (primary serial no. 002 and secondary serial no 001) passed their dynamics testing (vibration and shock) in November 2017 but the primary target failed its 


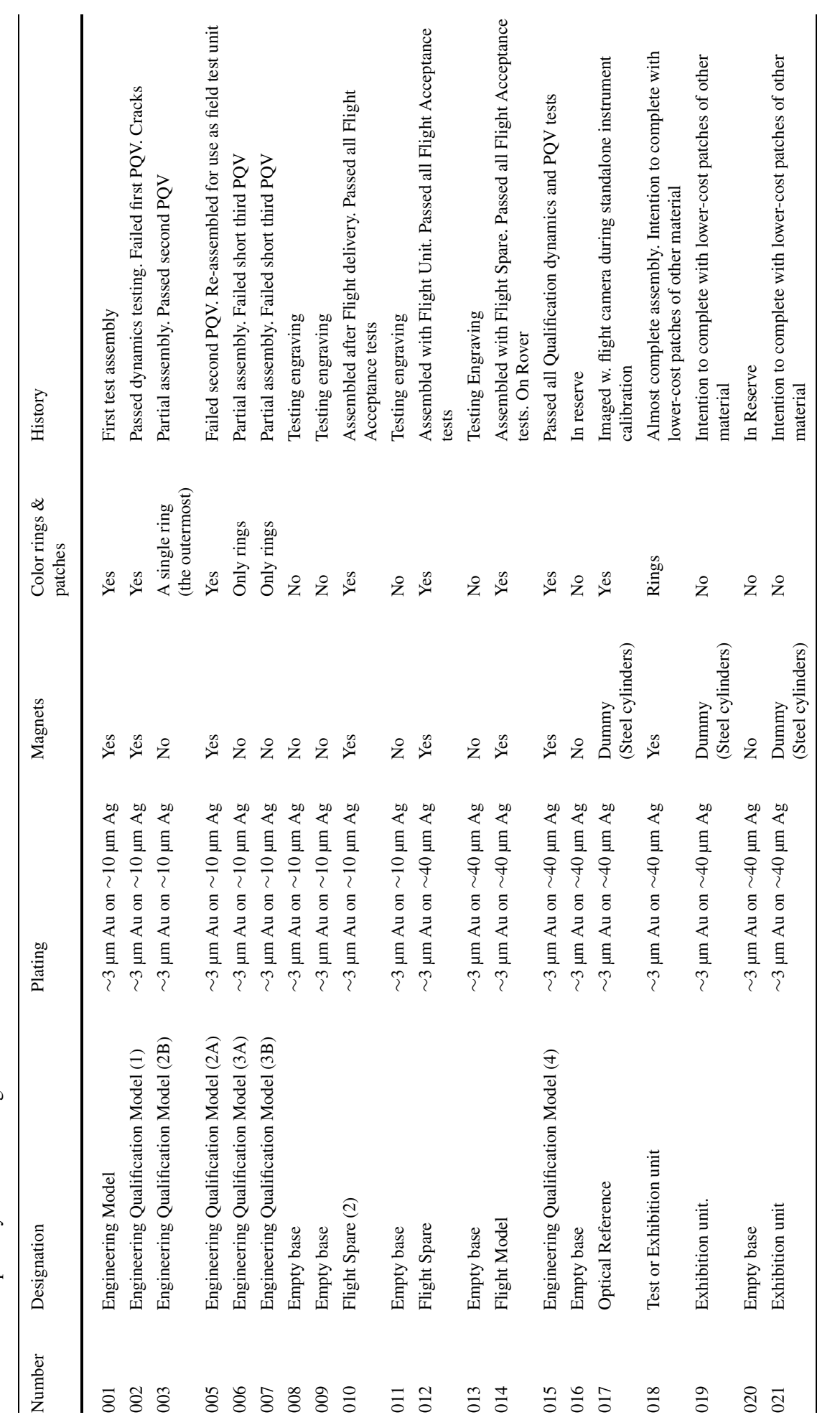


Table 6 List of secondary calibration target units

\begin{tabular}{lll}
\hline Number & Designation & History \\
\hline 001 & Engineering Qualification Model & $\begin{array}{l}\text { Passed all dynamics and PQV qualification tests with } \\
\text { primary \#002 }\end{array}$ \\
002 & Engineering Model & $\begin{array}{l}\text { First test assembly. Imaged w. flight camera during } \\
\text { standalone instrument calibration } \\
\text { Assembled with Flight Spare. Passed all Flight Acceptance } \\
\text { tests with primary \#014. On Rover } \\
\text { Assembled with Flight Model. Passed all Flight Acceptance } \\
\text { tests with primary \#012 }\end{array}$ \\
004 & Flight Model & $\begin{array}{l}\text { Passed all Flight Acceptance tests with primary \#010 } \\
\text { "Half shelf" base (i.e. } \text { only half of a target). Intended for } \\
\text { optical characterization }\end{array}$ \\
\hline
\end{tabular}

PQV test in the spring of 2018 developing cracks in the grayscale rings. This led to slight modifications to the design and to the type and application of epoxy and to several curtailed PQV tests during the summer and fall of 2018 on primary units serial no. 003, 005, 006 and 007 as described in Sect. B.2.1. These EQM units were not dynamically tested. Finally, a new primary EQM was produced (serial no. 015) and passed dynamics testing in November 2018 and PQV in early 2019.

The flight (primary serial no. 014, secondary serial no. 003) and flight spare targets (primary serial no. 012 and secondary serial no. 004) passed dynamics (i.e. vibration) testing immediately after the last EQM in late 2018. The flight targets passed TVAC in May 2019. The spares passed TVAC in late June 2019. After delivery of the flight targets a second set of flight spares were produced (primary serial no. 010 and secondary serial no. 005). These passed dynamics (vibration) testing in August 2019 and TVAC in September 2019.

All the tests are documented in detailed test reports. This section will just give a brief overview, with some description of the anomalies experienced in the first PQV tests but otherwise focusing on the qualification tests of the final EQM's and the flight acceptance tests of the flight units. The qualification level vibration test is described in Sect. B.1.1, the shock test in Sect. B.1.2 and the PQV test in Sect. B.2.1. The flight acceptance vibration test is described in Sect. B.1.3 and the TVAC test is described in Sect. B.2.2.

\section{B.1 Dynamics Tests}

Dynamics tests were all performed at Force Technology, Venlighedsvej 4, Hørsholm, Denmark. A total of one secondary target EQM and two primary target EQM's were subjected to the qualification level shock and vibration tests. A total of three sets of flight and flight spare units were subjected to the flight acceptance level vibration test. No anomalies were experienced in any of these tests. The reason for the repeat of the qualification test was the failed PQV test. Since we wanted to have an EQM unit that had passed the entire test program in sequence, that necessitated a repeat of the dynamics test. Below we give some detail on the final qualification test (on primary unit 015) and on the flight acceptance test on the flight units (secondary \#003 and primary \# 014). These tests were all performed during November 27th-30th, 2018. 
K.M. Kinch et al.

Table 7 Qualification vibration test spectrum

Fig. 25 EQM unit of Mastcam-Z primary calibration target (serial no. 015) mounted on vibration table in the "X-axis" configuration

\begin{tabular}{ll}
\hline Frequency range $(\mathrm{Hz})$ & Qualification test level \\
\hline $20-40$ & $+6.0 \mathrm{~dB} /$ octave \\
$40-450$ & $0.08 \mathrm{~g}^{2} / \mathrm{Hz}$ \\
$450-2000$ & $-6.0 \mathrm{~dB} /$ octave \\
Overall & $7.9 \mathrm{grms}$ \\
\hline
\end{tabular}

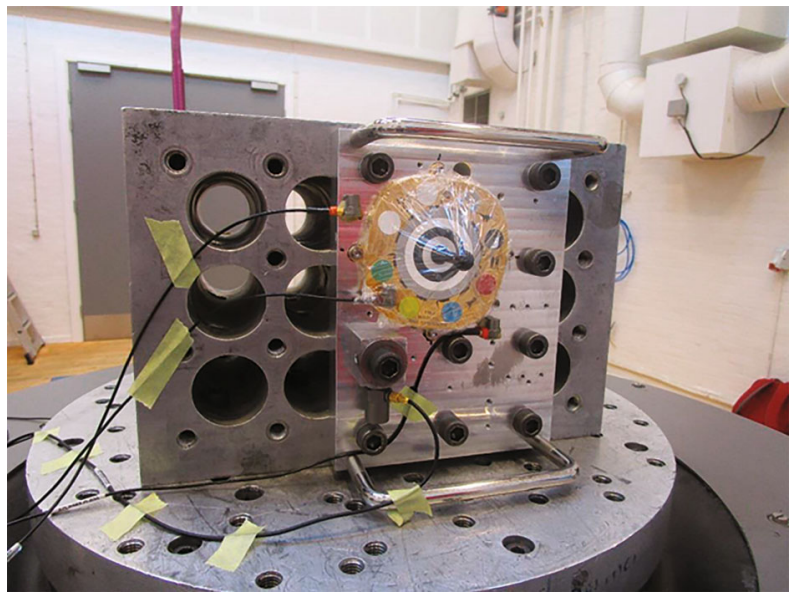

\section{B.1.1 Qualification Level Vibration}

The qualification level vibration test was performed to the specifications given in Table 7 and shown in Fig. 26. The vibration spectrum had an acceleration spectral density (ASD) between $40 \mathrm{~Hz}$ and $450 \mathrm{~Hz}$ of $0.08 \mathrm{~g}^{2} / \mathrm{Hz}$ and dropped off with $6.0 \mathrm{db} /$ octave to either side of this spectral region. The overall root mean square acceleration (square root of area under ASD curve) was $7.9 \mathrm{~g}$. The test duration was two minutes per axis.

Figure 25 shows the EQM unit serial no. 015 mounted on the vibration table in the "X-axis" configuration. The axes were named to be consistent with the axes of the rover coordinate system when the target is mounted on the rover. A total of four accelerometers were employed in the test apparatus; one control accelerometer and two redundant accelerometers were mounted to the test fixture itself, while one response accelerometer was mounted to the target. All accelerometer readings were consistent, confirming proper functioning of the instruments. Before and after vibration, a white-noise sweep of the target was performed to search for any new resonances perhaps indicating damage to the target. The only visible resonance was a known resonance in the fixture just below $2 \mathrm{kHz}$ (visible in Fig. 26). After that, a visual inspection of the target was performed to confirm that there was no damage. This procedure was repeated once for each axis.

\section{B.1.2 Qualification Level Shock}

Figure 27 shows the setup for the qualification shock test in the $\mathrm{X}$ direction. The axes were named to be consistent with the axes of the rover coordinate system when the target is mounted on the rover. The target is mounted to a fixture which is again fastened to a large and thick aluminum plate. The shock is generated by firing a steel nail gun into a small 


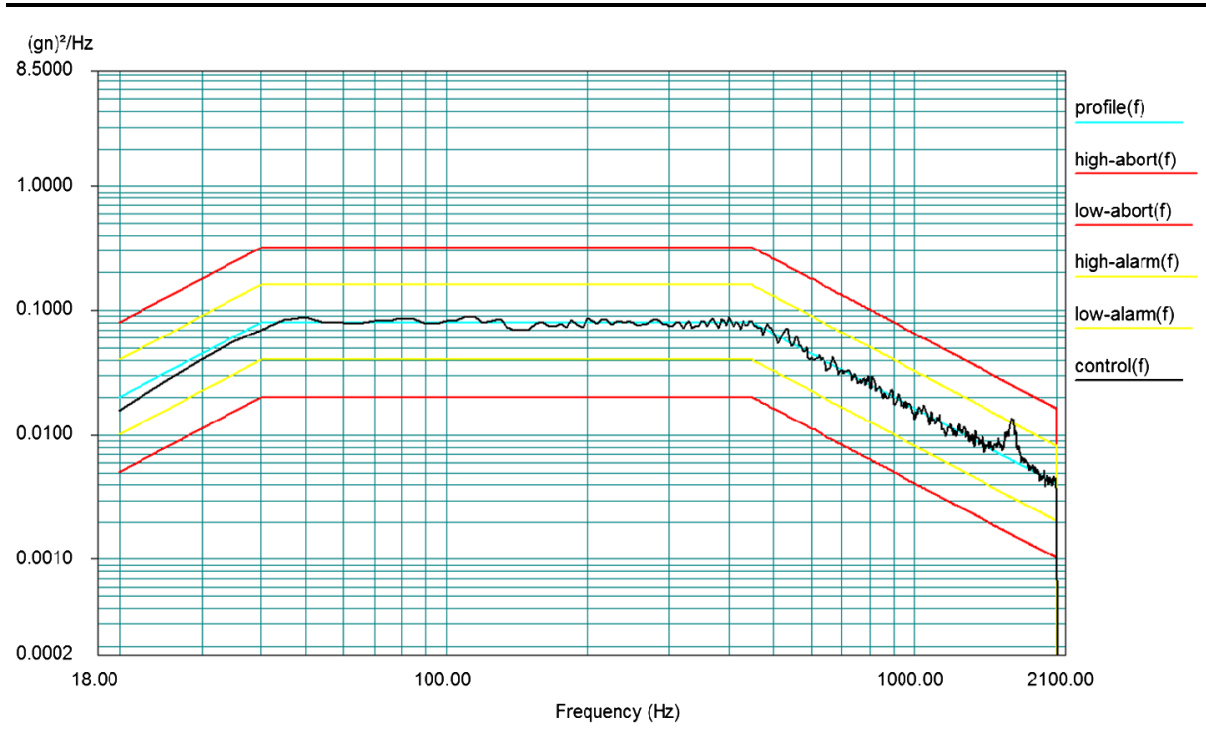

Fig. 26 Vibration spectrum measured from control accelerometer in the X-axis configuration (consistent with image in Fig. 25). Black curve is the measured spectrum, blue curve is the target spectrum. Yellow curves are $+/-3 \mathrm{~dB}$ bands around the target curve and red curves are $+/-6 \mathrm{~dB}$ bands around the target curve. The slight resonance peak in the measured (Black) curve just below $2 \mathrm{kHz}$ is a known resonance in the vibration table

Table 8 Flight Acceptance vibration test spectrum

\begin{tabular}{ll}
\hline Frequency range $(\mathrm{Hz})$ & Flight acceptance test level \\
\hline $20-40$ & $+6.0 \mathrm{~dB} /$ octave \\
$40-450$ & $0.04 \mathrm{~g}^{2} / \mathrm{Hz}$ \\
$450-2000$ & $-6.0 \mathrm{~dB} /$ octave \\
Overall & $5.6 \mathrm{~g}_{\mathrm{rms}}$ \\
\hline
\end{tabular}

block of soft aluminum firmly mounted at the opposite corner of the large aluminum plate. Two shocks were performed in each direction. Test data confirmed that the shocks were symmetric between the $+X$ and $-X$ direction and therefore separate shocks were not required between these two directions. The same was the case for $+/-Y$ and $+/-Z$. Figure 28 shows the time history of the first X-axis shock and Fig. 29 shows the "shock response spectrum" (SRS spectrum) of this same shock. The SRS spectrum shows that the X-axis shocks (blue curves) meet the requirements, except for some limited overshoot at low frequencies. These overshoots were reviewed and deemed acceptable, since overshoot means testing just a bit above requirements. This confirmed that a separate $-X$ test was not required. The orange $(-Z)$ curve does not meet requirements and accordingly separate shocks were performed in the $\mathrm{Y}$ direction. After each shock visual inspection confirmed integrity of the target.

\section{B.1.3 Flight Acceptance Level Vibration}

The flight acceptance level vibration test was performed to the specifications given in Table 8 and shown in Fig. 31. The vibration spectrum had an acceleration spectral density (ASD) between $40 \mathrm{~Hz}$ and $450 \mathrm{~Hz}$ of $0.04 \mathrm{~g}^{2} / \mathrm{Hz}$ and dropped of with $6.0 \mathrm{db}$ /octave to either side 


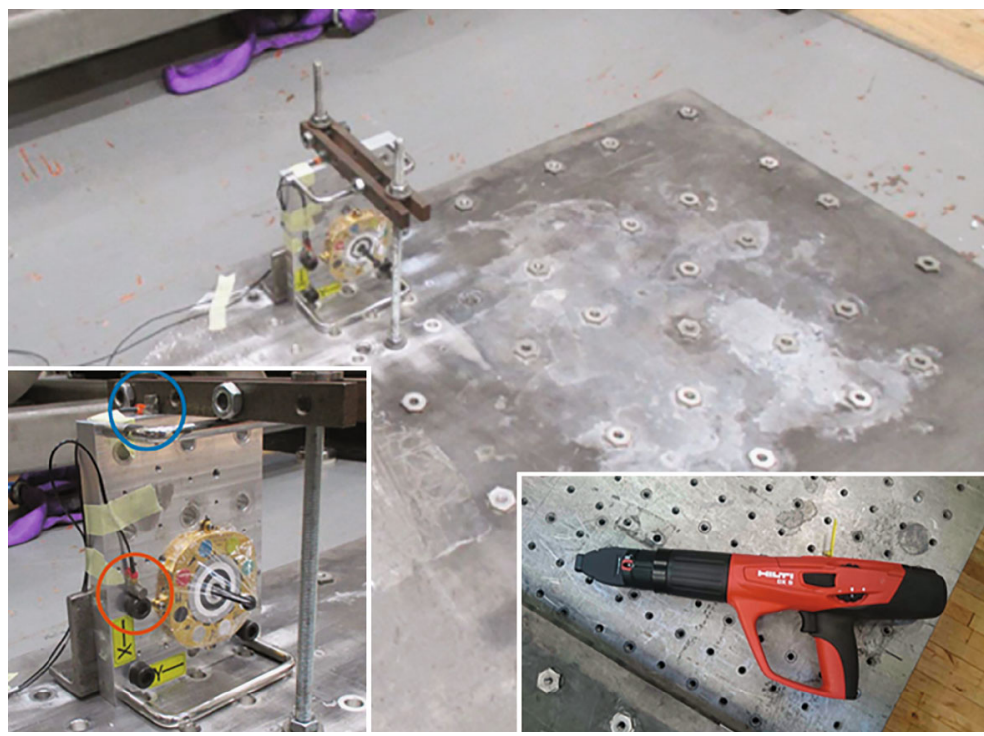

Fig. 27 EQM unit (serial no. 015) mounted on the shock test setup in the "X-axis" configuration. Insert bottom left shows a zoom-in on the target. The blue circle marks the location of the X-accelerometer. The orange-red circle marks the Z-accelerometer. Insert bottom right shows the Hilti nail gun used to generate the shock

of this spectral region. The overall root mean square acceleration (square root of area under ASD curve) was $5.6 \mathrm{~g}$. The test duration was one minute per axis.

Figure 30 shows the Flight units serial numbers 014 (primary) and 003 (secondary) mounted on the vibration table in the "X-axis" configuration. The axes were named to be consistent with the axes of the rover coordinate system after the target is mounted on the rover. A total of four accelerometers were employed: A control accelerometer bolted to the mounting fixture, a response accelerometer mounted on the primary target using wax, a response accelerometer mounted to the secondary target in the same way and one extra accelerometer mounted on the fixture again using wax. All accelerometer readings were consistent, confirming proper functioning of the instruments. Before and after vibration, a whitenoise sweep of the target was performed to search for any new resonances perhaps indicating damage to the target. The only visible resonance was a known resonance in the fixture just below $2 \mathrm{kHz}$ (visible in Fig. 31). After that, a visual inspection of the target was performed to confirm that there was no damage. This procedure was repeated once for each axis.

\section{B.2 Thermal Tests}

Thermal tests (PQV-tests) on EQM units were all performed at NASA Jet Propulsion Laboratory (JPL). Thermal-vacuum tests (TVAC-tests) on flight and flight spare units were performed at the Niels Bohr Institute, University of Copenhagen. Qualification PQV tests consisted of a large number (hundreds) of thermal cycles performed in an ambient pressure dry gaseous Nitrogen environment while flight acceptance TVAC consisted of three thermal cycles in vacuum. 
Shock \# $5 \mathrm{XZ}$ ch. A: X-axis ch. B: Z-axis

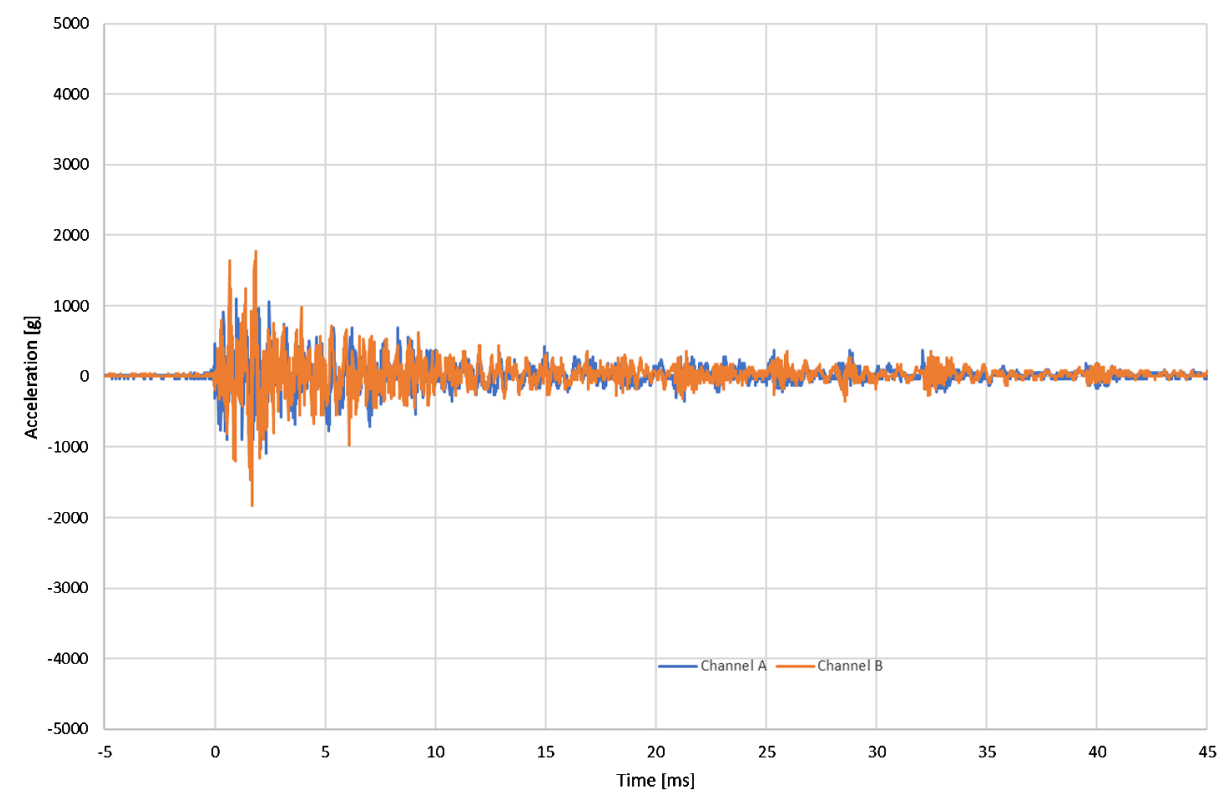

Fig. 28 Shock time history measured from shock in the X-axis configuration consistent with image in Fig. 27. The blue curve is the $\mathrm{X}$-direction accelerometer response. The orange curve is the signal from an accelerometer mounted in the Z-direction

Table 9 PQV test program, showing "workmanship" and "lifetime" cycles

\begin{tabular}{lcclllllllll}
\hline Cycle & Run & Count & $\begin{array}{l}\text { Cooling } \\
\text { rate } \\
(\mathrm{C} / \mathrm{min})\end{array}$ & $\begin{array}{l}\text { Low } \\
\text { Dwell } \\
\text { Temp } \\
(\mathrm{C})\end{array}$ & $\begin{array}{l}\text { Low } \\
\text { Dwell } \\
\text { Time } \\
(\mathrm{min})\end{array}$ & $\begin{array}{l}\text { Heating } \\
\text { rate } \\
(\mathrm{C} / \mathrm{min})\end{array}$ & $\begin{array}{l}\text { High } \\
\text { Dwell } \\
\text { Temp } \\
(\mathrm{C})\end{array}$ & $\begin{array}{l}\text { High } \\
\text { Dwell } \\
\text { Time } \\
(\mathrm{min})\end{array}$ & $\begin{array}{l}\text { Delta } \\
\mathrm{T}(\mathrm{C})\end{array}$ & $\begin{array}{l}\text { Cycle } \\
\text { time } \\
(\mathrm{min})\end{array}$ & $\begin{array}{l}\text { Time to } \\
\text { complete } \\
\text { (days })\end{array}$ \\
\hline Workmanship & 1 & 45 & -5 & -135 & 8 & 5 & 75 & 8 & 210 & 100 & 3.13 \\
1x Life & 2 & 120 & -5 & -135 & 8 & 5 & 75 & 8 & 210 & 100 & 8.33 \\
2x Life & 3 & 120 & -5 & -135 & 8 & 5 & 75 & 8 & 210 & 100 & 8.33 \\
$3 x$ Life & 4 & 120 & -5 & -135 & 8 & 5 & 75 & 8 & 210 & 100 & 8.33 \\
Totals & 405 & & & & & & & & & 28.13 \\
\hline
\end{tabular}

\section{B.2.1 PQV Tests}

The PQV test program was as outlined in Table 9. A total of 405 cycles were performed between $-135^{\circ} \mathrm{C}$ and $+75^{\circ} \mathrm{C}$ with 3 chamber breaks in between for inspection. Figure 32 shows the final EQM (serial \# 015) in the thermal chamber. The first set of 45 cycles are referred to as "workmanship" cycles. Often, design or construction flaws reveal themselves during this first part of the test. In addition to the 45 "workmanship" cycles, the PQV test consisted of 3 sets of 120 "lifetime" cycles. Because of the faster ramping and the wider temperature range, relative to conditions on the Martian surface, each of these sets of 120 cycles were estimated to result in equivalent wear to the target as the primary mission of one 


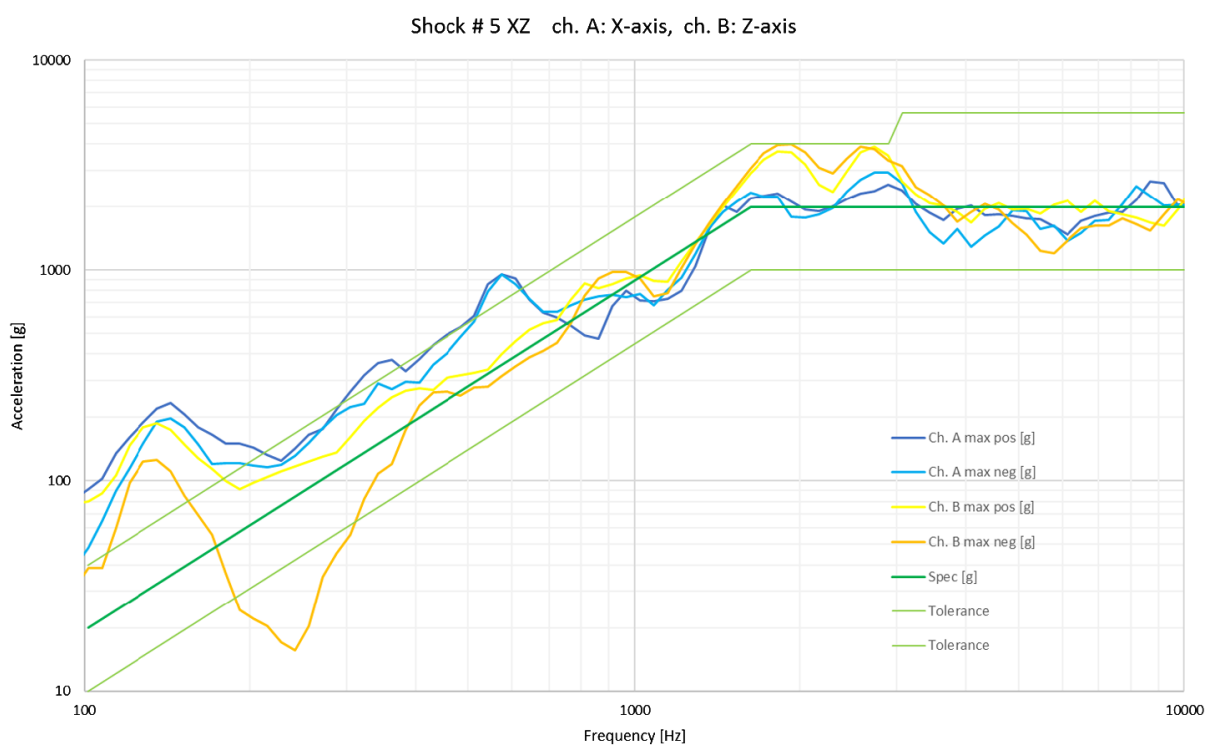

Fig. 29 Shock Response Spectrum (SRS) from shock in the X-axis configuration consistent with image in Fig. 27. These curves are calculated from the time histories in Fig. 11. Blue curves are for $+/-X$. Orange curves are $+/-Z$. The green curve shows the specified target and tolerances

Fig. 30 Flight units (serial number 014 and 003 ) mounted on vibration table in the "X-axis" configuration

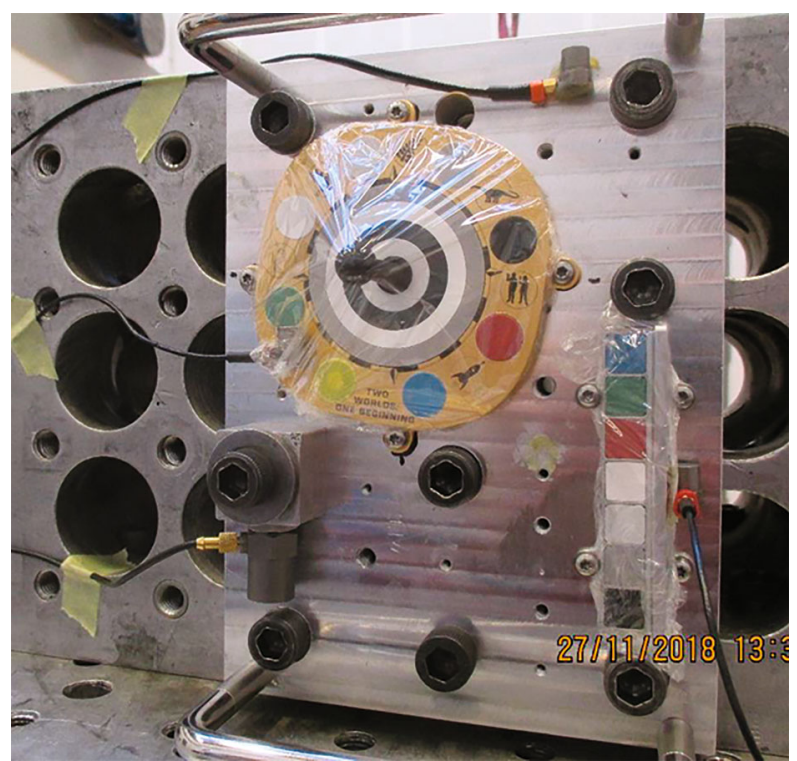

martian year. Figure 33 shows temperature curves over an example of cycles during the test of serial \# 015 .

When performing the first PQV test on the serial number 002 EQM unit cracks developed in the outer grayscale ring during workmanship cycling (see Fig. 34). The cause was determined to be stress related to the difference in thermal expansion between the aluminum 


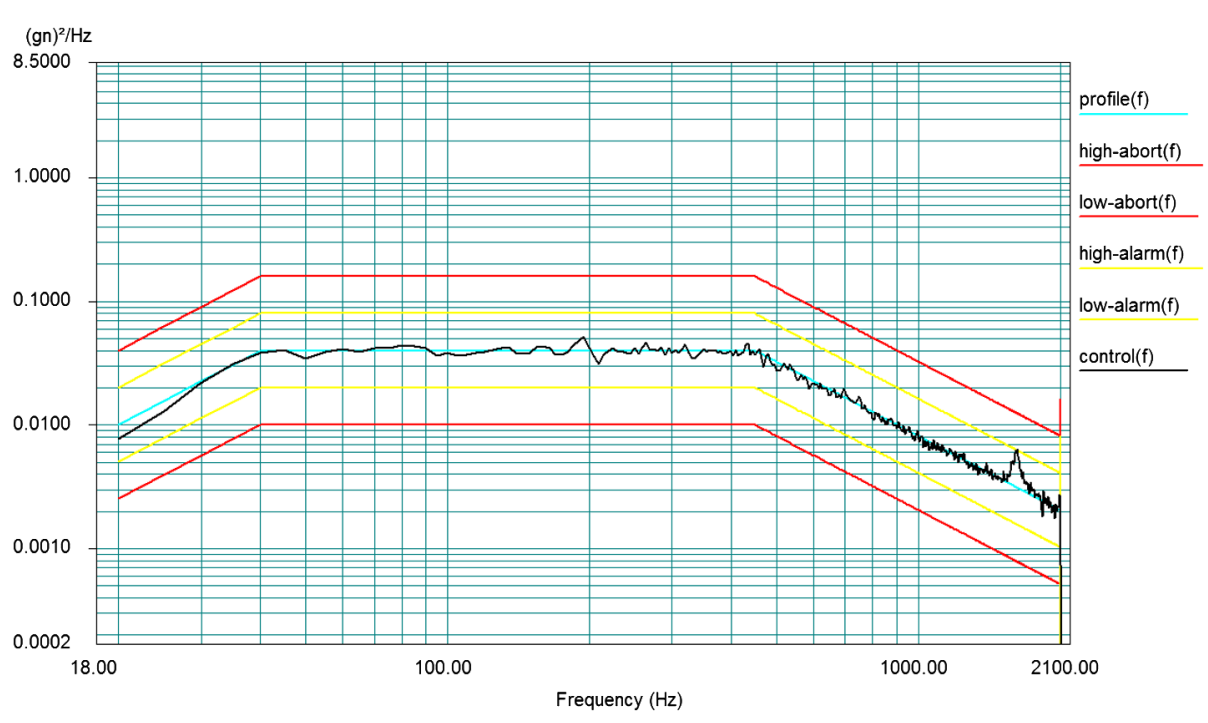

Fig. 31 Vibration spectrum measured from control accelerometer in the $\mathrm{X}$-axis configuration (consistent with image in Fig. 30). Black curve is the measured spectrum, blue curve is the target spectrum. Yellow curves are $+/-3 \mathrm{~dB}$ bands around the target curve and red curves are $+/-6 \mathrm{~dB}$ bands around the target curve. The slight resonance peak in the measured (Black) curve just below $2 \mathrm{kHz}$ is a known resonance in the vibration table

Fig. 32 The 4th EQM unit (serial \# 015) in the thermal chamber at NASA JPL in January 2019 during the passed PQV test

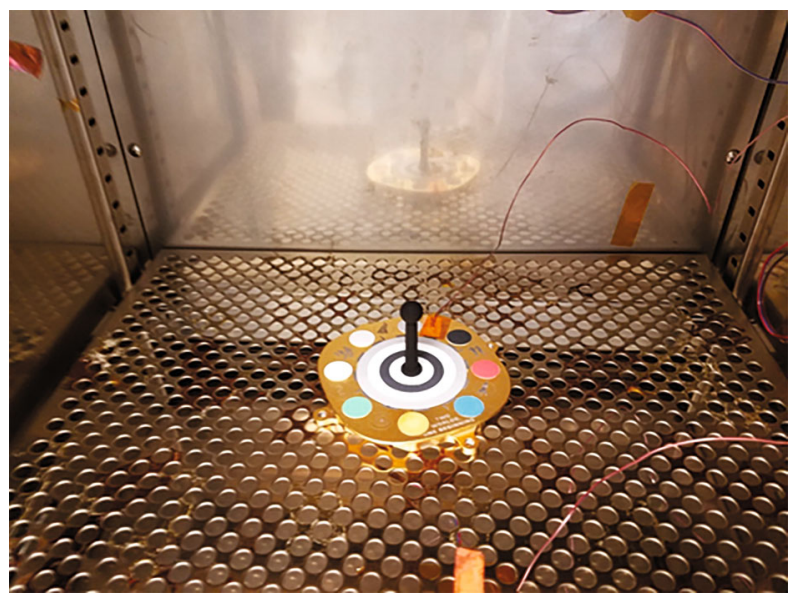

base and the ceramic rings. After several rounds of redesign and testing this was solved by 3 changes:

(1) A slight change to the dimensions of the base, widening the space on the outside of the outer ring. Introduction of Kapton ${ }^{\circledR}$ shims to ensure equal spacing along the periphery of rings.

(2) A different choice of epoxy for the grayscale rings, replacing Henkel/Loctite EA9309NA with $3 M 2216 B / A$, which is less stiff 


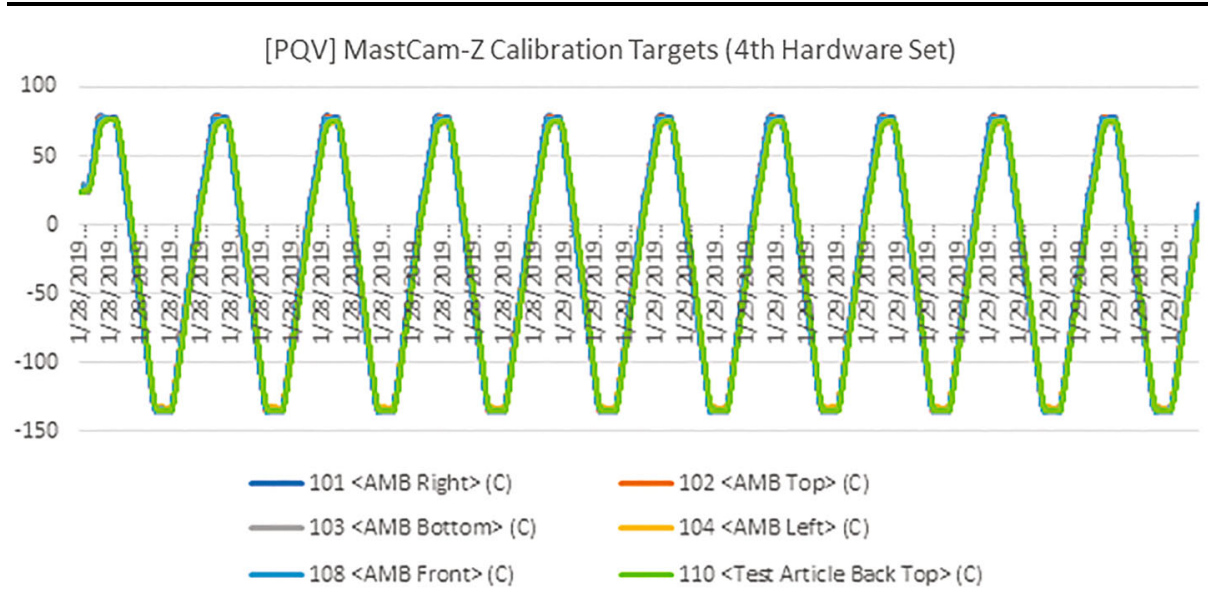

Fig. 33 Temperature curves over an example set of thermal cycles during the final PQV test of EQM 4, serial \# 015, in January 2019

Fig. 34 Cracks in the outer grayscale ring developed during the first PQV test (EQM unit serial number 002)

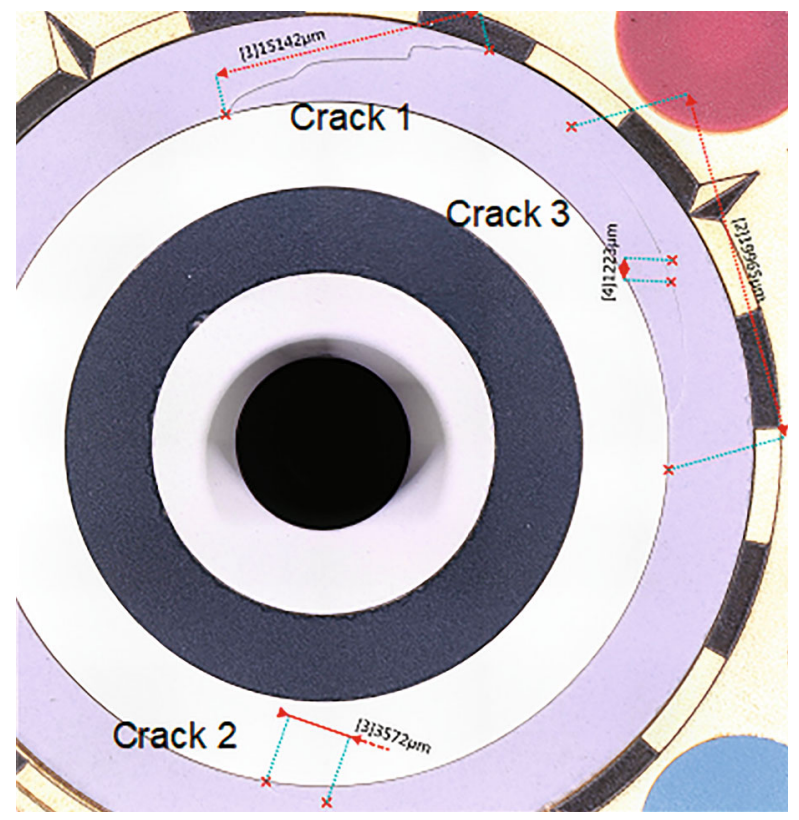

(3) Changes to the procedure for epoxy application, using more epoxy, applied as continuous bond lines, rather than dots, and mixing $1 \%$ by weight of $175 \mu \mathrm{m}$ spacer grade glass spheres with the epoxy to control bond line thickness.

For details of the final epoxy application procedure see Appendix A, Sect. A.1.5.

After these changes to the design, the new EQM unit (serial number 015) passed the full PQV test. High resolution before/after 3D-imaging revealed no significant observed changes to the structure. 

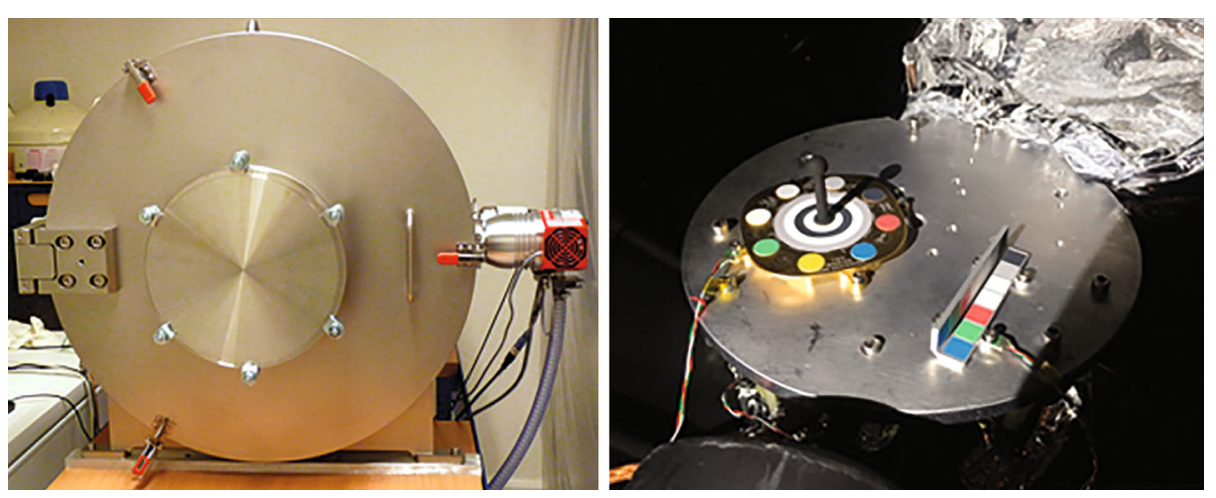

Fig. 35 Left: Chamber when closed with turbo pump to the right, Right: Inside of chamber with heating table, targets mounted and thermal shroud (open). Two Pt-100 temperature sensors with connecting wires mounted with the two targets are visible

Fig. 36 TVAC thermal and pressure history. The red "primary target" temperature curve is almost hidden under the orange "secondary target" temperature curve
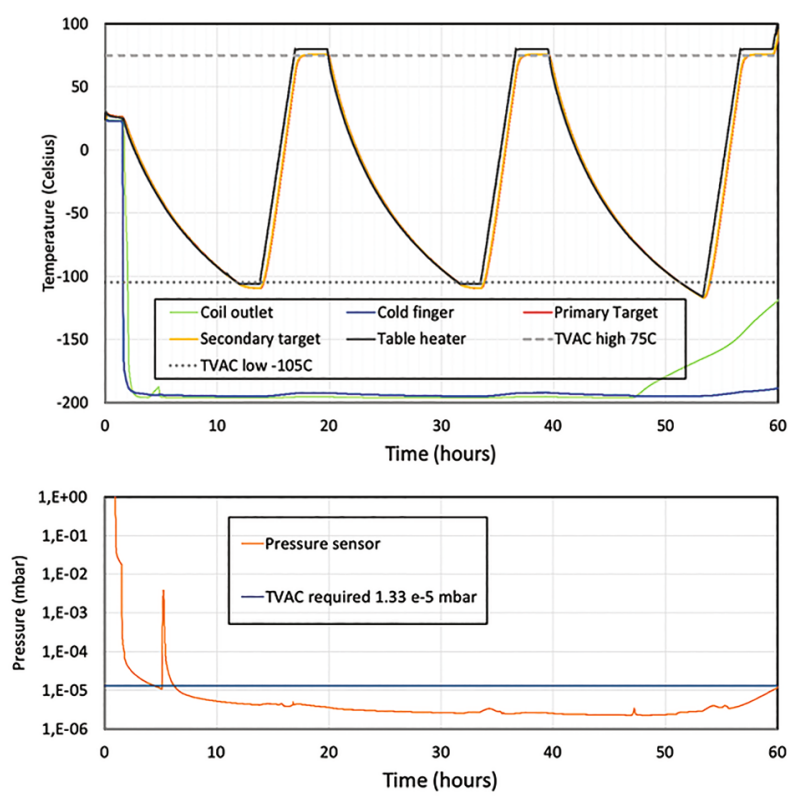

\section{B.2.2 TVAC Test}

The TVAC tests were performed at $<10^{-5}$ Torr $\left(<1.33 \cdot 10^{-5} \mathrm{mBar}\right)$. Three thermal cycles were performed between $+75^{\circ} \mathrm{C}$ and $-105^{\circ} \mathrm{C}$ with a 2-hour dwell time at each temperature extreme for each cycle, so a total dwell time of 6 hours at the $-105{ }^{\circ} \mathrm{C}$ and 6 hours at $+75^{\circ} \mathrm{C}$. Following the choice of Jezero Crater as Mars 2020 landing site the minimum test temperature was relieved from the $-135^{\circ} \mathrm{C}$ used during PQV tests to $-105^{\circ} \mathrm{C}$ reflecting the Jezero environment. Figure 35 shows the TVAC chamber with the flight targets mounted in the chamber.

Figure 36 shows the thermal and pressure history of the TVAC test on the flight units. The chamber was closed and the log begun at 10:16 AM on Friday the 10th of May 2019. 
The first low-temperature dwell was commenced in the evening of that day. The last lowtemperature dwell happened around noon on Sunday the 12th and the last high-dwell happened in the evening of Sunday the 12th, completing the TVAC sequence. Temperature was continually logged with 5 Pt-100 temperature sensors. 2 bolted to each of the two targets (visible in Fig. 35 right), one mounted on the underside of the table near the heater (control sensor) and two mounted on the cooling system, one at the outlet at the far end of the cooling tubes ("coil outlet") and one on the cold finger close to the inlet ("cold finger"). In addition, the pressure was continually logged with a Pfeiffer Vacuum Compact FullRangeTM Gauge PKR 251 pressure sensor. The temperature and pressure curves complied with the specifications for the test.

After TVAC cycling the targets were baked at $>110{ }^{\circ} \mathrm{C}$ for 48 hours. Subsequently the chamber was opened and the targets were briefly inspected before packed in a neutral atmosphere for delivery to NASA JPL. During this inspection no anomalies were discovered.

At the receiving inspection at JPL some small $(<1 \mathrm{~mm})$ blisters were discovered on the underside of the target. Subsequent re-inspection of EQM units revealed similar blistering on the last EQM (\#015), which had experienced a similar bakeout, but not on previous EQM units, although these were also baked. This led to the idea that the units with the thicker $(\sim 40 \mu \mathrm{m})$ silver layer are prone to develop these blisters when baked at $>110{ }^{\circ} \mathrm{C}$, but the units with the thinner $(\sim 10 \mu \mathrm{m})$ silver layer do not have this weakness. For this reason, the flight spare (\#012, thick silver layer) was only baked at $75{ }^{\circ} \mathrm{C}$, and did not develop such blisters. Later, a second flight spare was produced using one of the thinner silver layer bases (\#010). This unit was baked at $>110^{\circ} \mathrm{C}$ and developed no blistering. Both flight spares also passed the full dynamics and TVAC program with no issues.

After careful review at JPL it was determined that the adhesion of the silver and gold plating was still very good on the flight target, despite the small blisters. It was therefore decided NOT to replace the flight unit with a spare. The underside of the flight unit was painted with a thin polyurethane coating as an extra precaution.

\section{References}

J.F. Bell III, S.W. Squyres, K.E. Herkenhoff et al., Mars Exploration Rover Athena Panoramic Camera (Pancam) investigation. J. Geophys. Res. 108(E12), 8063 (2003)

J.F. Bell III et al., In-flight calibration and performance of the Mars Exploration Rover Panoramic Camera (Pancam) instruments. J. Geophys. Res. 111, E02S03 (2006). https://doi.org/10.1029/2005JE002444

J.F. Bell III et al., The Mars Science Laboratory Curiosity Rover Mast Camera (Mastcam) instruments: Preflight and in-flight, calibration, validation, and data archiving. Earth Space Sci. 4, 396-452 (2017). https://doi.org/10.1002/2016EA00021

J.F. Bell III, J.N. Maki, G.L. Mehall et al., The Mars 2020 Perseverance Rover Mast Camera Zoom (Mastcam-Z) Multispectral, Stereoscopic Imaging Investigation. Space Sci. Rev. 217, 24 (2021). https:// doi.org/10.1007/s11214-020-00755-x

P. Bertelsen, W. Goetz, M.B. Madsen et al., Magnetic properties experiments on the Mars exploration rover spirit at Gusev crater. Science 305(5685), 827-829 (2004)

D.D. Bogard et al., Consideration of Sample Return and the Exploration Strategy for Mars (1979). http://ntrs. nasa.gov/archive/nasa/casi.ntrs.nasa.gov/19790019931.pdf

J. Buz et al., Photometric characterization of Lucideon and Avian Technologies color standards including application for calibration of the Mastcam-Z instrument on the Mars 2020 rover. Opt. Eng. 58(2), 027108 (2019). https://doi.org/10.1117/1.oe.58.2.027108

C.S. Cockell, D.C. Catling, W.L. Davis, K. Snook, R.L. Kepner, P. Lee, C.P. McKay, The ultraviolet environment of Mars: biological implications past, present, and future. Icarus 146(2), 343-359 (2000). https://doi.org/10.1006/icar.2000.6393

M. Craig, E.A. Cloutis, T. Mueller, ME and mini-ME: Two Mars environmental simulation chambers for reflectance spectroscopy, in Lunar Planet. Sci. Conference, vol. XXXII, Abstract 1368 (2001) 
L. Drube et al., Magnetic and optical properties of airborne dust and settling rates of dust at the Phoenix landing site. J. Geophys. Res. 115, E00E23 (2010). https://doi.org/10.1029/2009JE003419

K. Farley et al., Mars 2020 Mission overview. Space Sci. Rev. (2020, this issue). https://doi.org/10.1007/ s11214-020-00762-y

J.P. Grotzinger et al., A habitable fluvio-lacustrine environment at Yellowknife Bay, Gale crater, Mars. Science 343, 1242777 (2014)

J.P. Grotzinger et al., Deposition, exhumation, and paleoclimate of an ancient lake deposit, Gale crater, Mars. Science 350, aac7575 (2015)

B. Hapke, Theory of Reflectance and Emittance Spectroscopy, edited by R. E. Arvidson and M. J. Rycroft (Cambridge Univ. Press, Cambridge, 1993)

R.B. Hargraves et al., Viking magnetic properties investigation: Further results. Science 194, 1303-1309 (1976). https://doi.org/10.1126/science.194.4271.1303

R.B. Hargraves et al., Viking magnetic properties experiment: Extended mission results. J. Geophys. Res. 84, 8379-8384 (1979). https://doi.org/10.1029/JB084iB14p08379

A.G. Hayes, P. Corlies, C. Tate et al., Pre-Flight Calibration of the Mars 2020 Rover Mastcam Zoom (Mastcam-Z) Multispectral, Stereoscopic Imager. Space Sci. Rev. 217, 29 (2021). https://doi.org/10. 1007/s11214-021-00795-x

J. Hollandt, U. Becker, W. Paustian et al., New developments in the radiance calibration of deuterium lamps in the UV and VUV spectral range at the PTB. Metrologia 37(5), 563 (2000)

F.O. Huck, G.R. Taylor, H.F. McCall et al., The Viking Mars lander camera. Space Sci. Instrum. 1, 189-241 (1975)

J.R. Johnson et al., Radiative transfer modeling of dust-coated Pancam calibration target materials: Laboratory visible/near-infrared spectrogoniometry. J. Geophys. Res., Planets 111, E12S07 (2006). https://doi. org/10.1029/2005JE002658

J.R. Johnson, J.F. Bell III, S. Bender et al., ChemCam passive reflectance spectroscopy of surface materials at the curiosity landing site, Mars. Icarus 249, 74-92 (2015)

K.M. Kinch, J.M. Merrison, H.P. Gunnlaugsson et al., Preliminary analysis of the MER magnetic properties experiment using a computational fluid dynamics model. Planet. Space Sci. 54, 28-44 (2006)

K.M. Kinch, M.B. Madsen, J.F. Bell III et al., Dust on the curiosity mast camera calibration target, LPSC-44 \#1061 (2013)

K.M. Kinch, J.F. Bell III, W. Goetz et al., Dust deposition on the decks of the Mars Exploration Rovers: 10 years of dust dynamics on the Panoramic Camera calibration targets. Earth Space Sci. (2015). https://doi.org/10.1002/2014EA000073

K. Kinch, M. Hilverda, M.B. Madsen, J. Bell, C. Mars, The Planetary Report 40(4) (2020)

K. Leer, P. Bertelsen, C.S. Binau et al., Magnetic Properties Experiments and the SSI calibration target onboard the Mars Phoenix 2007 Lander. Design, calibration and science goals. J. Geophys. Res. 113, E00A16 (2008). https://doi.org/10.1029/2007JE003014

M.B. Madsen, W. Goetz, P. Bertelsen et al., Overview of the magnetic properties experiments on the Mars Exploration Rovers. J. Geophys. Res. 114(E6), E06S90 (2009). https://doi.org/10.1029/2008je003098

J.A. Manrique et al., SuperCam calibration target design. One target for a complete analytical suite. Space Sci. Rev. (2020, this issue). https://doi.org/10.1007/s11214-020-00764-w

S. Maurice et al., The SuperCam instrument suite on the Mars 2020 rover: Science objectives and Mast-Unit description. Space Sci. Rev. (2020, this issue). Manuscript number: SPAC-D-20-00069R1

M.R. Patel, J.C. Zarnecki, D.C. Catling, Ultraviolet radiation on the surface of Mars and the Beagle 2 UV sensor. Planet. Space Sci. 50(9), 915-927 (2002). https://doi.org/10.1016/S0032-0633(02)00067-3

A. Pommerol et al., Photometry and bulk physical properties of Solar System surfaces icy analogs: The Planetary Ice Laboratory at University of Bern. Planet. Space Sci. 59, 1601-1612 (2011)

M.K. Shepard, P. Helfenstein, A test of the Hapke photometric model. J. Geophys. Res., Planets 112, E03001 (2007). https://doi.org/10.1029/2005JE002625

P.H. Smith et al., The Imager for Mars Pathfinder experiment. J. Geophys. Res. 102, 4003-4025 (1997)

D. Wellington et al., Visible to near-infrared MSL/Mastcam, multispectral imaging: Initial results from selected high-interest science targets within Gale crater, Mars. Am. Mineral. 102(6), 1202-1217 (2017). https://doi.org/10.2138/am-2017-5760CCBY

R. Wiens et al., The SuperCam instrument suite on the NASA Mars 2020 rover: Body unit and combined system tests. Space Sci. Rev. (2020, this issue) Manuscript no: SPAC-D-20-00065

W. Zdunkowski, T. Trautmann, A. Bott, Radiation in the Atmosphere, 1st edn. (Cambridge University Press, Cambridge, 2007) 